\title{
15. Sur la théorie des hypersurfaces dans un espace à connexion conforme.
}

\author{
Par Kentaro Yano et Yosio Mutô. \\ (reçu le 10 Juin, 1940.)
}

\section{Introduction.}

On sait que c'est M. E. Cartan $\left({ }^{1}\right)$ qui a premièrement introduit la notion de connexion conforme et a développé la théorie des espaces à connexion conforme avec sa méthode du repère mobile. Il s'est aussi occupé des sous-espaces dans cet espace à connexion conforme.

D'autre part, les géomètres de l'Ecole de Princeton, $\left({ }^{2}\right)$ en particulier MM. O.Veblen, T. Y. Thomas et J. M. Thomas ont étudié, d'après l'idée fondamentale de M. H. Weyl, les invariants conformes d'une forme quadratique $d s^{2}=g_{j k} d u^{j} d u^{k}$, c'est-à-dire, les quantités invariantes par rapport à la transformation conforme

$$
g_{j k} \rightarrow \rho^{2} g_{j k}
$$

du tenseur fondamental $g_{j k}$ d'un espace de Riemann.

Pour étudier la géométrie conforme généralisée, les géomètres de l'Ecole de Delft, $\left({ }^{3}\right)$ MM. J. A. Schouten et J. Haantjes ont proposé encore une autre méthode qui consiste à employer les coordonnées homogènes de M. D. van Dantzig.

Tout récemment, un des présents auteurs $\left({ }^{4}\right)$ a étudié la théorie des espaces à connexion conforme à l'aide du repère mobile naturel de M. E. Cartan et a examiné en détail la relation entre la théorie de M. E. Cartan et celle de l'Ecole de Princeton.

(1) E. Cartan (I). Voir la Bibliographie placée à la fin du présent Mémoire. Nous avons acceuilli ici seulement les Mémoires sur la théorie des sous-espaces dans un espace à connexion conforme. On peut trouver une Bibliographie complète sur la théorie générale des espaces à connexion conforme à la fin du Mémoire de K. Yano (2).

(2) T. Y. Thomas: Differential invariants of generalized spaces. Cambridge University Press. 1935.

O. Veòien: Formalism for conformal geometry. Proc. Nat. Acad. Sci. 21 (1935) $168-173$.

(3) J. A. Schouten et J. Haantjes : Beiträge zur allgemeinen (gekrümmten) konformen Differentialgeometrie. I. II. Math. Ann. 112 (1936) 594-629, 113 (1936) 568-583.

(4) K. Yano (2). 
Il a étudié aussi la théorie des courbes et en particulier la théorie des circonférences généralisées $\left({ }^{1}\right)$ dans cet espace à connexion conforme.

Le but du présent Mémoire est de développer la théorie des hypersurfaces dans un espace à connexion conforme. La méthode adoptée ici est celle de M.E. Cartan utilisée dans le Mémoire cité ci-dessus de K. Yano. $\left({ }^{2}\right)$

Dans le première Chapitre, nous résumerons, pour faciliter la lecture, quelques résultats obtenus dàns le Mómoire de K. Yano.

Dans le deuxième Chapitre, on considère une hypersurface dans un espace à connexion conforme et étudie les repères attachés à cette hypersurface.

Le Chapitre 3 est consacré à la détermination de la connexion conforme sur l'hypersurface par rapport à la connexion conforme de l'espace ambiant.

On étudie, dans le Chapitre 4, les tenseurs de courbure de l'hypersurface et cherche les équations de Gauss et de Codazzi bien connues dans le cas de l'espace de Riemann. Dans le Chapitre 5, on traite des courbes tracées sur l'hypersurface et en particulier dans le Chapitre 6 les circonférences généralisées tracées sur l'hypersurface.

Le Chapitre 7 est consacré à l'étude des courbes autoconcourantes considérées par M. A. Haimovici. $\left({ }^{3}\right)$

On étudie finalement, dans le Chapitre 8, quelques propriétés des ombilics et des hypersurfaces totalement ombiliquées.

Table des Matières

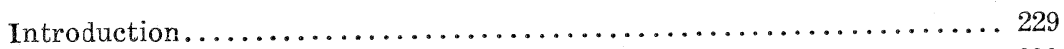

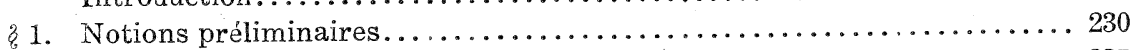

8. Les hypersurfaces dans un espace à connexion conforme.......... 237

8. 3. La connexion conforme induite sur les hypersurfaces............ 246

\&. 4. Tenseurs de courbure, les équations de Gauss et de Codazzi ........ 260

8. Propriétés des courbes sur les hypersurfaces................. 270

\& 6. Circonférences généralisées sur les hypersurfaces............. 277

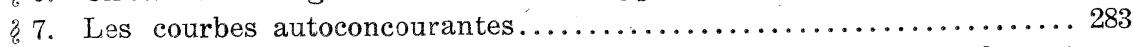

\&. Les propriétés des ombilics et des hypersurfaces totalement

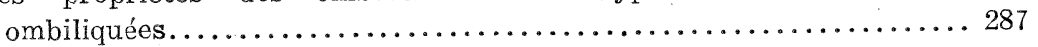

\section{Chapitre I. Notions préliminaires.}

Prenons une variété arithmétique à $n$ dimensions, c'est-à-dire une variété numérique dont chaque point est défini par un système de $n$ nombres réels $u^{1}, u^{2}, \ldots, u^{n}$ et supposons que le voisinage immédiat

(1) K. Yano (1), (2).

(2) K. Yano (2).

(3) A. Haimovici (1). 
de chaque point de la variété présente toutes les propriétés de l'espace conforme ordinaire. Si l'on a une loi qui nous permet d'accorder les espaces conformes ordinaires à deux points infiniment voisins de la variété, on peut accorder deux espaces conformes à une distance finie en les accordant de proche en proche le long d'une courbe donnée. Nous appellerons une telle variété la variété à connexion conforme. La correspondance conforme entre ces deux espaces conformes dépend généralement de la courbe qui relie deux points auxquels sont attachés les espaces conformes ordinaires. Si cette correspondance ne dépend pas de la courbe, l'espace à connexion conforme se réduit à un espace conforme ordinaire.

Cela dit, prenons une variété à connexion conforme. Dans chaque espace conforme attaché à un point courant $A_{0}$ (espace conforme tangent à $\left.A_{0}\right)$, on prend un repére mobile $(n+2)$-sphérique formé avec deux points analytiques $A_{0}$ et $A_{\infty}$ (considérés comme étant deux sphères du rayon nul) et $n$ sphères analytiques $A_{i}(i, j, k, \ldots=1,2,3, \ldots, n)$ passant par $A_{0}$ et $A_{\infty}$, alors on aura

$$
A_{0} A_{0}=A_{\infty} A_{\infty}=A_{0} A_{i}=A_{\infty} A_{i}=0 .
$$

Les sphères $A_{0}, A_{i}$ et $A_{\infty}$ étant déterminées à un facteur près, on peut choisir ce facteur de manière qu'on ait

$$
A_{0} A_{\infty}=-1
$$

Les produits des sphères $A_{j}$ et $A_{k}$

$$
A_{j} A_{k}=g_{j k}
$$

sont, en général, les fonctions de coordonnées $u^{i}$.

Cela, étant, la connexion conforme peut être exprimée par les formules de la forme:

$$
\left\{\begin{aligned}
d A_{0} & =\omega_{0}^{0} A_{0}+\omega_{0}^{1} A_{1}+\cdots+\omega_{0}^{n} A_{n}+\omega_{0}^{\infty} A_{\infty}, \\
d A_{1} & =\omega_{1}^{0} A_{0}+\omega_{1}^{1} A_{1}+\cdots+\omega_{1}^{n} A_{n}+\omega_{1}^{\infty} A_{\infty}, \\
& \cdots \cdots+\cdots \cdots+\omega_{n}^{n} A_{n}+\omega_{n}^{\infty} A_{\infty}, \\
d A_{n} & =\omega_{n}^{0} A_{0}+\omega_{n}^{1} A_{1}+\cdots+\omega_{\infty}^{n} A_{n}+\omega_{\infty}^{\infty} A_{\infty}, \\
d A_{\infty} & =\omega_{\infty}^{0} A_{0}+\omega_{\infty}^{1} A_{1}+\cdots
\end{aligned}\right.
$$

où les coefficients de $A_{0}, A_{i}$ et $A_{\infty}$ sont des formes de Pfaff par rapport aux coordonnées $u^{i}$. 
Or, les équations (1.1), (1.2) et (1.3) nous donnent

$$
\left\{\begin{array}{c}
\omega_{0}^{\infty}=\omega_{\infty}^{0}=0, \omega_{0}^{i} g_{i j}-\omega_{j}^{\infty}=0, \omega_{\infty}^{i} g_{i j}-\omega_{j}^{0}=0, \omega_{0}^{0}+\omega_{\infty}^{\infty}=0, \\
\omega_{j}^{i} g_{i k}+\omega_{k}^{i} g_{i j}=d g_{j k} .
\end{array}\right.
$$

$\omega_{0}^{\infty}$ étant identiquement nul, $d A_{0}$ a la forme suivante:

$$
d A_{0}=\omega_{0}^{0} A_{0}+\omega_{0}^{1} A_{1}+\cdots+\omega_{0}^{n} A_{n} .
$$

Cela étant, quand on effectue une transformation du repère

$$
\bar{A}_{B}=U_{B}^{C} A_{C}, \quad(A, B, C, \ldots=0,1,2, \ldots, n, \infty)
$$

$\omega_{0}^{0}$ et $\omega_{0}^{i}$ seront respectivement transformés par les équations

$$
\left\{\begin{aligned}
U_{0}^{0} \bar{\omega}_{0}^{0}+U_{i}^{0} \bar{\omega}_{0}^{i} & =U_{0}^{0} \omega_{0}^{0}+d U_{0}^{0}, \\
U_{\jmath}^{i} \bar{\omega}_{0}^{j} & =U_{0}^{0} \omega_{0}^{i}
\end{aligned}\right.
$$

Ces équations nous assurent qu'on peut choisir un repère $(n+2)$. sphérique pour lequel on a

$$
\omega_{0}^{0}=p_{i} d u^{i}, \quad \omega_{0}^{i}=d u^{i},
$$

c'est-à-dire qu'on a, pour le déplacement de $A_{0}$,

$$
d A_{0}=d u^{0} A_{0}+d u^{1} A_{1}+\cdots+d u^{n} A_{n}
$$

et par conséquent

$$
\left(d A_{0}\right)^{2}=g_{i j} d u^{i} d u
$$

où nous avons posé

$$
d u^{0}=p_{i} d u^{i}
$$

On appelle repère mobile semi-naturel un repère mobile réalisant la condition (1.10) et par suite (1.11)

La connexion conforme étant complètement définie par

$$
\left\{\begin{array}{l}
\Pi_{B 0}^{A}=\delta_{B}^{A} \\
\Pi_{B k}^{A}=\omega_{B k}^{A}-\delta_{B}^{A} p_{k}
\end{array}\right.
$$

où

$$
\omega_{B k}^{A} d u^{k}=\omega_{B}^{A},
$$

nous appelons $\Pi_{B 0}^{A}$ et $\Pi_{B k}^{A}$ les composantes de la connexion conforme. 
Dans le cas de sans torsion, on obtient de (1.5)

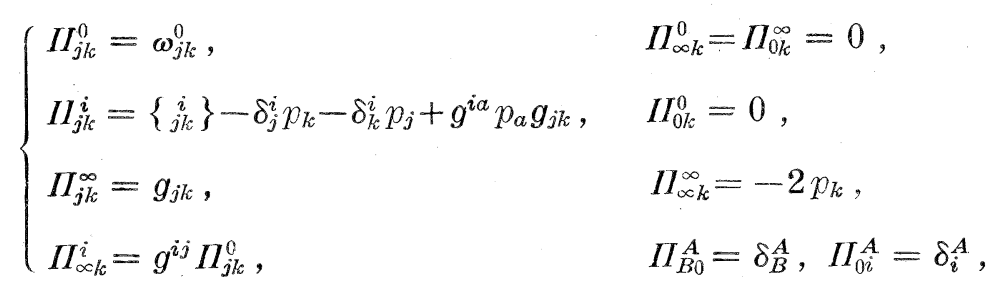

où les $\left\{\begin{array}{l}i \\ j k\end{array}\right\}$ désignent les symboles de Christoffel formés avec les $g_{j k}$,

$$
\left\{\begin{array}{l}
i \\
j k
\end{array}\right\}=\frac{1}{2} g^{i a}\left(g_{a j, k}+g_{a k, j}-g_{j k, a}\right),
$$

la virgule désignant la dérivée partielle par rapport à $u^{k}$.

Le choix du repère mobile semi-naturel n'étant pas unique, on peut admettre encore deux sortes de transformations du repère semi-naturel:

(i) transformation du tenseur fondamental

$$
\left\{\begin{array}{l}
\bar{A}_{0}=\lambda A_{0} \\
\bar{A}_{i}=\lambda A_{i} \\
\bar{A}_{\infty}=\frac{1}{\lambda} A_{\infty},
\end{array}\right.
$$

(ii) transformation du point de l'infini

$$
\left\{\begin{array}{l}
\bar{A}_{0}=A_{0}, \\
\bar{A}_{i}=\phi_{i} A_{0}+A_{i}, \\
\bar{A}_{\infty}=\frac{1}{2} g_{i j} \phi^{i} \phi^{j} A_{0}+\phi^{i} A_{i}+A_{\infty}, \quad\left(\phi^{i}=g^{i j} \phi_{j}\right),
\end{array}\right.
$$

où $\lambda$ et $\phi_{i}$ sont des fonctions arbitraires de coordonnées $u^{k}$.

Quand on effectue ces transformations du repère semi-naturel, les composantes de la connexion conforme se transforment de la manière suivante:

Pour la transformation $\mathrm{du}$ tenseur fondamental, on a

$$
\left\{\begin{array}{l}
\bar{p}_{k}=p_{k}+\lambda_{k}, \\
\bar{I}_{j k}^{0}=I I_{j k}^{0}, \\
\bar{I}_{j k}^{i}=I_{j k}^{i}, \\
\bar{\Pi}_{i j}^{\infty}=\lambda^{2} \Pi_{i j}^{\infty}, \\
\bar{\Pi}_{\infty k}^{i}=\frac{1}{\lambda^{2}} I_{\infty k}^{i},
\end{array}\right.
$$


ou

$$
\lambda_{k}=\frac{\partial \log \lambda}{\partial u^{k}}
$$

et pour la transformation du point de l'infini, on a

$$
\left\{\begin{array}{l}
\bar{l}_{k}=p_{k}-\phi_{k}, \\
\bar{I}_{j k}^{0}=I_{j k}^{0}+\left(\phi_{j, k}-\phi_{i} \Pi_{j k}^{i}\right)-\phi_{j} \phi_{k}+\frac{1}{2} g_{a b} \phi^{a} \phi^{b} g_{j k}, \\
\bar{\Pi}_{j k}^{i}=\Pi_{j k}^{i}+\delta_{j}^{i} \phi_{k}+\delta_{k}^{i} \phi_{j}-g^{i a} \phi_{a} g_{j k} \\
\bar{\Pi}_{j k}^{\infty}=I_{j k}^{\infty} \\
\bar{\Pi}_{\propto k}^{i}=I_{\propto k}^{i}+\left(\phi^{i}, k+\phi^{j} I I_{j k}^{i}\right)-\phi^{i} \phi_{k}+2 \phi^{i} p_{k}+\frac{1}{2} g_{a b} \phi^{a} \phi^{b} \delta_{k}^{i} .
\end{array}\right.
$$

En utilisant ces deux sortes de transformation, on peut uniquement déterminer un repère semi-naturel particulier satisfaisant aux conditions supplémentaires suivantes:

$$
\left\{\begin{array}{c}
p_{k}=0 \\
\Pi_{i k}^{i}=0 .
\end{array}\right.
$$

Dans ce cas, nous aurons, pour les composantes de la connexion, l'expression suivante

$$
\left\{\begin{array}{l}
\Pi_{0 k}^{0}=I_{\infty k}^{0}=I_{0 k}^{\infty}=I_{\infty k}^{\infty}=0, \quad I_{0 j}^{i}=\delta_{j}^{i}, \\
\Pi_{j k}^{i}=\frac{1}{2} G^{i a}\left(G_{a j, k}+G_{a k, j}-G_{j k, a}\right), \\
I_{j k}^{\infty}=G_{j k}, \\
I_{j k}^{0}=G_{i j} \Pi_{\infty}^{i},
\end{array}\right.
$$

où $G_{j k}$ est une densité tesorielle du poids $-\frac{2}{n}$ définie par

$$
A_{i} A_{j}=G_{i j}=g_{i j} / g^{\frac{1}{n}}
$$

$g$ étant le déterminant formé avec les $g_{i j}$. Il est à remarquer que la valeur du déterminant $\left|G_{i j}\right|$ est égale à l'unité.

Nous appelons repère naturel le repère semi-naturel satisfaisant à la condition supplémentaire (1.21).

Quand on fixe le système de coordonnées de la variété, le repère mobile naturel sera uniquement déterminé. Cela étant, quand on effectue une transformation de coordonnées 


$$
\bar{u}^{i}=\bar{u}^{i}\left(u^{1}, u^{2}, u^{3}, \ldots, u^{n}\right)
$$

avec le jacobien $\Delta$ non nul

$$
J=\left|\frac{\partial u}{\partial \bar{u}}\right| \neq 0
$$

les $A_{0}, A_{j}$ et $A_{\infty}$, formant le repère naturel, seront transformés en $\bar{A}_{0}, \bar{A}_{s}$ et $\bar{A}_{\infty}$ respectivement de la manière suivante:

$$
\left\{\begin{array}{l}
\bar{A}_{0}=j^{-\frac{1}{n}} A_{0}, \\
\bar{A}_{i}=J^{-\frac{1}{n}} \bar{\psi}_{i} A_{0}+J^{-\frac{1}{n}} \frac{\partial u^{j}}{\partial \bar{u}^{i}} A_{j}, \\
\bar{A}_{\infty}=\frac{1}{2} J^{-\frac{1}{n}} \bar{G}^{i j} \bar{\psi}_{i} \bar{\psi}_{j} A_{0}+J^{-\frac{1}{n}} \bar{G}^{i j} \bar{\psi}_{i} \frac{\partial u^{a}}{\partial \bar{u}^{j}} A_{a}+J^{+\frac{1}{n}} A_{\infty},
\end{array}\right.
$$

où

$$
\bar{\psi}_{i}=\frac{\partial \log J^{-\frac{1}{n}}}{\partial \bar{u}^{i}},
$$

et

$$
\left\{\begin{array}{l}
\bar{G}_{i j}=J^{-\frac{2}{n}} \frac{\partial u^{a}}{\partial \bar{u}^{i}} \frac{\partial u^{b}}{\partial \bar{u}^{j}} G_{a b} \\
\bar{G}^{i j}=J^{+\frac{2}{n}} \frac{\partial \bar{u}^{i}}{\partial u^{a}} \frac{\partial \bar{u}^{j}}{\partial u^{b}} G^{a b}, \quad G^{a b} G_{b c}=\delta_{c}^{a}
\end{array}\right.
$$

Le tenseur de courbure étant défini par

$$
\Omega_{B k h}^{A}=\Pi_{B l, h}^{A}-\Pi_{B h, k}^{A}+\Pi_{B l c}^{C} \Pi_{C h}^{A}-\Pi_{B h}^{C} \Pi_{C k}^{A},
$$

le tenseur

$$
C_{j k h}^{i}=\Omega_{j k h}^{i}-\delta_{j}^{i} \Omega_{0 k h}^{n}
$$

joue un rôle très important dans la théorie des espaces à connexion conforme.

Quand le tenseur $C_{j k h}^{i}$ satifait à la condition

$$
C_{j k i}^{i}=0,
$$

la connexion sera dite normale. Si la connexion est normale, le tenseur de courbure $C_{j k h}^{i}$ coïncide avec celui de $\mathrm{M}$. Weyl. 
Dans ce cas, on peut choisir, parmi les repères mobiles semi-naturels, un repère privilégié pour lequel les composantes de la connexion seront:

$$
\left\{\begin{array}{l}
\Pi_{0 i}^{0}=\Pi_{\infty i}^{\infty}=\Pi_{\infty i}^{0}=\Pi_{0 i}^{\infty}=0, \quad \Pi_{0 j}^{i}=\delta_{j}^{i}, \\
\Pi_{j k}^{0}=-\frac{R_{j k}}{n-2}+\frac{g_{j k} R}{2(n-1)(n-2)}, \\
\Pi_{j k}^{i}=\left\{\begin{array}{c}
i \\
j k
\end{array}\right\}=\frac{1}{2} g^{i a}\left(g_{a j, k}+g_{a k, j}-g_{j k, a}\right) \\
\Pi_{j k}^{\infty}=g_{j k}, \\
\Pi_{\infty c k}^{i}=-\frac{R_{k k}^{i}}{n-2}+\frac{\delta_{k}^{i} R}{2(n-1)(n-2)}
\end{array}\right.
$$

où

$$
\begin{aligned}
& R_{j k}=R_{j k i}^{i}, \quad R_{k}^{i}=g^{i j} R_{j k}, \quad R=g^{i j} R_{i j}, \\
& \text { et } \quad R_{j k}^{i}=\left\{\begin{array}{c}
i \\
j k
\end{array}\right\}, h-\left\{\begin{array}{c}
i \\
j h
\end{array}\right\}, k+\left\{\begin{array}{c}
a \\
j k
\end{array}\right\}\left\{\begin{array}{c}
i \\
a h
\end{array}\right\}-\left\{\begin{array}{c}
a \\
j h
\end{array}\right\}\left\{\begin{array}{c}
i \\
a k
\end{array}\right\} .
\end{aligned}
$$

Nous appelons ce repère le repère de M. O. Veblen. $\left({ }^{1}\right)$

Dans le cas de la connexion normale, les composantes de la connexion par rapport au repère naturel seront:

$$
\left\{\begin{array}{l}
\Pi_{B 0}^{A}=\delta_{B}^{A}, \quad I_{0 j}^{i}=\delta_{j}^{i}, \quad \Pi_{0 k}^{0}=\Pi_{\propto k}^{\infty}=I_{\propto k}^{0}=I_{0 k}^{\infty}=0, \\
\Pi_{j k}^{0}=-\frac{1}{n-2}\left[\Pi_{j k i}^{i}-\frac{G_{j k} \Pi_{a b i}^{i} G^{a b}}{2(n-1)}\right], \\
\Pi_{j k}^{i}=\frac{1}{2} G^{i a}\left(G_{a j, k}+G_{a k, j}-G_{j k, a}\right), \\
I_{j k}^{\infty}=G_{j k}, \\
I_{\propto k}^{i}=G^{i j} I_{j k}^{0},
\end{array}\right.
$$

où

$$
I_{j k h}^{i}=\Pi_{j k, h}^{i}-I I_{j h, k}^{i}+I I_{j k}^{a} I I_{a h}^{i}-\Pi_{j h}^{a} \Pi_{a k}^{i}
$$

La connexion que nous étudierons dans la suite n'étant pas toujours normale, nous employons en principe le repère naturel, mais si la connexion est normale, nous utiliserons aussi le repère de M. O. Veblen.

(1) O. Veblen: Formalism for conformal geometry. Proc. Nat. Acad. Sci. 21 (1935) 168-173. 


\section{Chapitre 2. Les hypersurfaces dans un espace $\dot{\alpha}$ connexion conforme.}

On considère une hypersurface $C_{n-1}$ dans une variété $C_{n}$ à connexion conforme telle que nous avons expliquée dans le Chapitre précédent. Une telle hypersurface sera donnée par les équations paramétriques de la forme

$$
u^{\dot{i}}=u^{i}\left(u^{\dot{1}}, u^{\dot{2}}, u^{\dot{3}}, \ldots, u^{\dot{n}-1}\right)
$$

ou plus simplement par

$$
u^{i}=u^{i}\left(u^{\alpha}\right), \quad(\alpha=1, \dot{z}, \ldots, \dot{n}-i) .
$$

Nous supposerons, dans la suite, que les lettres lattines $i, j, k, \cdots$ parcourent les symboles $1,2,3, \cdots, n$ tandis que les lettres grecques $\alpha, \beta, \gamma, \cdots$ parcourent les symboles $1, \dot{2}, \dot{3}, \cdots, n-\dot{1}$. A chaque point $\left(u^{\alpha}\right)$ sur cette hypersurface, nous attacherons un espace conforme ordinaire à $(n-1)$ dimensions et nous allons considérer sa connexion. On peut déterminer une connexion sur cette hypersurface à partir de la densité tensorielle fondamentale sur l'hypersurface, mais ce que nous allons faire dans la suite, c'est déterminer une connexion sur l'hypersurface par rapport à la connexion conforme de la variété dans laquelle est plongée cette hypersurface.

Etant donnée une connexion de la variété ambiante $C_{n}$, nous allons choisir un repère $\left[A_{\dot{0}}, A_{\alpha}, A_{\dot{n}}, A_{\dot{\infty}}\right]$ sur notre hypersurface. $A_{\dot{0}}$ sera un point sur l'hypersurface, il est, bien entendu, regardé aussi comme ètant un point de l'espace ambiant $C_{n}$.

Alors, $A_{\dot{0}}$ doit avoir la forme

$$
A_{0}=\rho A_{0},
$$

où $\rho$ est une fonction arbitraire des paramètres $u^{\alpha}$.

Quand on considère un point $\left(u^{i}+\frac{\partial u^{i}}{\partial u^{\alpha}} d u^{\alpha}\right)$ infiniment voisin $d u$ point $\left(u^{i}\right)$ sur l'hypersurface, le $A_{\dot{0}}$ considéré à ce point correspond à $A_{\dot{0}}+d A_{\dot{0}}$ dans l'espace conforme attaché au point $\left(u^{i}\right)$, et on aura

$$
\begin{aligned}
d A_{0} & =d \rho A_{0}+\rho d A_{0} \\
& =\frac{\partial \rho}{\partial u^{\alpha}} d u^{\alpha} A_{0}+\rho \frac{\partial u^{i}}{\partial u^{\alpha}} d u^{\alpha} A_{i} .
\end{aligned}
$$


Donc, si l'on veut choisir $A_{\alpha}$ de manière qu'on ait

$$
d A_{0}=d u^{\alpha} A_{\alpha},
$$

on doit poser

$$
A_{\alpha}=\frac{\partial \rho}{\partial u^{\alpha}} A_{0}+\rho \frac{\partial u^{i}}{\partial u^{\alpha}} A_{i} .
$$

Les $A_{0}$ et $A_{\alpha}$ sont bien déterminés ainsi à une fonction arbitraire $\rho$ près, si l'on se donne un système de coordonnées $\left(u^{\alpha}\right)$ sur l'hypersurface.

L'interprétation géométrique de $A_{0}$ est évidente. $A_{0}$ étant un point courant de la variété $C_{n}, A_{0}$ est un point courant sur l'hypersurface. Quant à $A_{\mathrm{i}}$, par exemple, on peut l'interpréter comme il suit: si l'on se déplace sur la courbe le long de laquelle le seul paramètre $u^{\mathrm{i}}$ change, on a

$$
A_{0}+d A_{0}=A_{0}+d u^{i} A_{1},
$$

on voit done que $A_{1}$ est une sphère qui passe par le point $A_{0}$ et orthogonale à la direction de la tangente de la courbe le long de laquelle $u^{\mathrm{i}}$ seul change.

Nous allons choisir $A_{i}$ comme une sphère orthogonale à toutes les sphères $A_{\alpha}$ passant par le point $A_{0}$. Alors, nous avons toujours

$$
d A_{\dot{0}} A_{\dot{n}}=0
$$

sur l'hypersurface, donc on voit que $A_{i}$ est une sphère tangente à l'hypersurface. Si l'on normalise $A_{\dot{n}}$, on aura

$$
A_{\dot{n}} A_{\dot{n}}=1,
$$

mais, il reste encore une transformation de $A_{\dot{n}}$ de la forme

$$
A_{\dot{n}} \rightarrow \sigma A_{\dot{0}}+A_{\dot{n}} .
$$

La sphère $A_{n}$ satisfaisant aux relations

$$
A_{j} A_{\dot{n}}=0, \quad A_{\alpha} A_{\dot{n}}=0, \quad A_{\dot{n}} A_{\dot{n}}=1,
$$

si, l'on définit une densité vectorielle $N^{i}$ du poids $+\frac{1}{n}$ par les équations

$$
G_{i j} \frac{\partial u^{i}}{\partial u^{\alpha}} N^{j}=0, \quad G_{j k} N^{j} N^{k}=1,
$$


on aura une équation de la forme

$$
A_{\dot{n}}=N^{0} A_{0}+N^{i} A_{i}
$$

oi $N^{0}$ est une fonction indéterminée. Il est évident que $\left(N^{0}, N^{i}, 0\right)$ forment les composantes d'un vecteur conforme contrevariant( ${ }^{1}$ ) par rapport au repére naturel.

On désigne finalement par $A_{\dot{\infty}}$ le point d'intertection autre que $A_{0}$ de sphères $A_{\alpha}$ et $A_{n}$, et on le normalise de manière qu'on ait

$$
A_{i} A_{\infty}=-1
$$

Alors $A_{\infty}$ peut être représenté par l'équation suivante

$$
\begin{aligned}
A_{\infty}=\left[\frac{1}{2}\left\{\frac{1}{\rho}\left(N^{0}\right)^{2}+\rho G^{\alpha \beta} \frac{\partial \log \rho}{\partial u^{\alpha}} \frac{\partial \log \rho}{\partial u^{\beta}}\right\} A_{0}\right. \\
\left.+\left(\frac{1}{\rho} N^{0} N^{i}+\rho G^{\alpha \beta} \frac{\partial u^{i}}{\partial u^{\alpha}} \frac{\partial \log \rho}{\partial u^{\beta}}\right) A_{i}+\frac{1}{\rho} A_{\infty}\right],
\end{aligned}
$$

où

$$
G_{\alpha_{3}}=A_{\alpha} A_{\beta}=\rho^{2} G_{i j} \frac{\partial u^{i}}{\partial u^{\alpha}} \frac{\partial u^{j}}{\partial u^{j}}, \quad G^{\alpha_{\beta}} G_{\beta \gamma}=\delta_{\gamma}^{\alpha} .
$$

Nous avons pu ainsi déterminé complètement un point $A_{0}$ sur l'hypersurface, $n-1$ sphères $A_{\alpha}$ passant par $A_{0}$ et orthogonale aux toutes les courbes paramétriques sur l'hypersurface, une sphère $A_{n}$ passant par $A_{1}$ et orthogonale aux $A_{\propto}$ et le point d'intersection $A_{\infty}$ des $A_{\dot{0}}, A_{\alpha}$ et $A_{\dot{\alpha}}$.

Ils comprennent cepandant encore deux fonctions arbitraires $\rho$ et $N^{0}$. Pour déterminer d'abord la fonction $\rho$, nous imposons la condition suivante:

$$
\operatorname{det}\left|A_{\alpha} A_{\beta}\right|=\operatorname{det}\left|G_{\alpha_{3}}\right|=1 \text {. }
$$

Alors la fonction $\rho$ sera déterminée par l'équation

$$
\rho^{2(n-1)} G_{i j} \frac{\partial u^{i}}{\partial u^{\alpha}} \frac{\partial u^{j}}{\partial u^{\beta}}=1 .
$$

Nous allons chercher une autre expression pour le déterminant $G_{i j} \frac{\partial u^{i}}{\partial u^{\alpha}} \frac{\partial u^{j}}{\partial u^{\beta}}$. Pour cela, posons

(1) K. Yano (2) p. 26. 
$(2.10)$

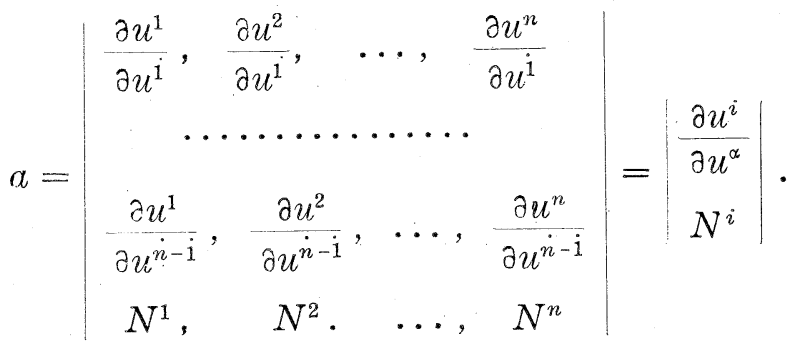

Alors, par la loi de multiplication des déterminants, on aura

$$
a \cdot\left|G_{i j}\right| \cdot a=\left|\begin{array}{rr}
G_{i j} \frac{\partial u^{i}}{\partial u^{\alpha}} \frac{\partial u^{j}}{\partial u^{\beta}}, & 0 \\
0, & 1
\end{array}\right|=\left|G_{i j} \frac{\partial u^{i}}{\partial u^{\alpha}} \frac{\partial u^{j}}{\partial u^{\beta}}\right|,
$$

par conséquent, on obtiendra

$$
\rho=a^{-\frac{1}{n-1}}
$$

grâce à l'identité $\left|G_{i j}\right|=1$.

La fonction $\rho$ étant ainsi déterminée, nous avons de (2.3), (2.4), (2.8) et (2.9)

$$
\begin{aligned}
& A_{0}=a^{-\frac{1}{n-1}} A_{0}, \\
& A_{\alpha}=a^{-\frac{1}{n-1}}\left(\frac{\partial \log a^{-\frac{1}{n-1}}}{\partial u^{\alpha}} A_{0}+\frac{\partial u^{i}}{\partial u^{\alpha}} A_{i}\right) \text {, } \\
& A_{n}=N^{0} A_{0}+N^{i} A_{i} \text {, } \\
& A_{\dot{\infty}}=a^{\frac{1}{n-1}}\left[\frac{1}{2}\left\{\left(N^{0}\right)^{2}+a^{-\frac{2}{n-1}} G^{\alpha \beta} \frac{\partial \log a^{-\frac{1}{n-1}}}{\partial u^{\alpha}} \frac{\partial \log a^{-\frac{1}{n-1}}}{\partial u^{\beta}}\right\} A_{0}\right. \\
& \left.+\left\{N^{0} N^{i}+a^{-\frac{2}{n-1}} G^{\alpha_{\beta}} \frac{\partial u^{i}}{\partial u^{\alpha}} \frac{\partial \log a^{-\frac{1}{n-1}}}{\partial u^{\beta}}\right\} A_{i}+A_{\infty}\right] \\
& =\frac{1}{2} a^{-\frac{1}{n-1}}\left[\left(a^{\frac{1}{n-1}} N^{0}\right)^{2}+G^{\alpha \beta} \frac{\partial \log a^{-\frac{1}{n-1}}}{\partial u^{\alpha}} \frac{\partial \log a^{-\frac{1}{n-1}}}{\partial u^{\beta}}\right] A_{0} \\
& +a^{-\frac{1}{x-1}}\left[\left(a^{\frac{1}{n-1}} N^{0}\right)\left(a^{\frac{1}{n-1}} N^{i}\right)\right. \\
& \left.+G^{\alpha \beta} \frac{\partial u^{i}}{\partial u^{\alpha}} \frac{\partial \log a^{-\frac{1}{n-1}}}{\partial u^{\beta}}\right] A_{i}+a^{\frac{1}{n-1}} A_{\infty} .
\end{aligned}
$$


Quand on effectue une transformation du système de coordonnées $\left(u^{i}\right)$ de l'espace ambiant, les $A_{0}, A_{i}$, et $A_{\infty}$ se transforment d'après

$$
\left\{\begin{array}{l}
\bar{A}_{0}=J^{-\frac{1}{n}} A_{0}, \\
\bar{A}_{i}=J^{-\frac{1}{n} \frac{\partial \log J^{-\frac{1}{n}}}{\partial \bar{u}^{i}} A_{0}+\Delta^{-\frac{1}{n}} \frac{\partial u^{j}}{\partial \bar{u}^{i}} A_{j}} \\
\bar{A}_{\infty}=\frac{1}{2} \Delta^{-\frac{1}{n}} \bar{G}^{i j} \frac{\partial \log \Delta^{-\frac{1}{n}}}{\partial \bar{u}^{i}} \frac{\partial \log \Delta^{-\frac{1}{n}}}{\partial \bar{u}^{j}} A_{0} \\
+J^{-\frac{1}{n}} \bar{G}^{i j} \frac{\partial u^{a}}{\partial \bar{u}^{i}} \frac{\partial \log \Delta^{-\frac{1}{n}}}{\partial \bar{u}^{j}} A_{a}+\Delta^{\frac{1}{n}} A_{\infty}
\end{array}\right.
$$

ou

$$
\left.\bar{G}^{i j}=\right\lrcorner^{+\frac{2}{n}} \frac{\partial \bar{u}^{i}}{\partial u^{\alpha}} \frac{\partial \bar{u}^{j}}{\partial u^{b}} G^{a b},
$$

et les $N^{0}$ et $N^{i}$ d'après

$$
\left\{\begin{array}{l}
\bar{N}^{0}=\Delta^{\frac{1}{n}} N^{0}-J^{\frac{1}{n}} \frac{\partial \log \Delta^{-\frac{1}{n}}}{\partial \bar{u}^{j}} \frac{\partial \bar{u}^{j}}{\partial u^{a}} N^{a} \\
\bar{N}^{i}= \\
J^{\frac{1}{n}} \frac{\partial \bar{u}^{i}}{\partial u^{j}} N^{j} .
\end{array}\right.
$$

Quand au déterminant $a$, il se transforme suivant

$$
\bar{a}=\left|\begin{array}{l}
\frac{\partial \bar{u}^{i}}{\partial u^{\alpha}} \\
\bar{N}^{i}
\end{array}=\right| \begin{aligned}
& \frac{\partial \bar{u}^{i}}{\partial u^{j}} \frac{\partial u^{j}}{\partial u^{\alpha}} \\
& \Delta^{\frac{1}{n}} \frac{\partial \bar{u}^{i}}{\partial u^{j}} N^{j}
\end{aligned}\left|=\Delta^{\frac{1}{n}} \cdot\right| \frac{\partial \bar{u}^{i}}{\partial u^{j}}|\cdot| \begin{aligned}
& \frac{\partial u^{j}}{\partial u^{\alpha}} \\
& N^{j}
\end{aligned}
$$

c'est-à-dire suivant

$$
\bar{a}=j^{-\frac{n-1}{n}} a
$$

On voit donc facilement que les $A_{0}, A_{\alpha}, A_{\dot{n}}$ et $A_{\infty}$ restent invariants pendant une transformation de système de coordonnées de l'espace ambiant. 
Cela étant, considérons une transformation de coordonnées de l'hypersurface

$$
\bar{u}^{\alpha}=\bar{u}^{\alpha}\left(u^{\beta}\right)
$$

et posons

$$
D=\frac{\partial u^{\alpha}}{\partial \bar{u}^{\beta}}
$$

Dans ce cas, le déterminant $a$ se transforme par

$$
\bar{a}=\left|\begin{array}{c}
\frac{\partial u^{i}}{\partial \bar{u}^{\beta}} \\
N^{i}
\end{array}\right|=\left|\begin{array}{c}
\frac{\partial u^{i}}{\partial u^{\alpha}} \frac{\partial u^{\alpha}}{\partial \bar{u}^{\beta}} \\
N^{i}
\end{array}\right|,
$$

c'est-à-dire par

$$
\bar{a}=D \cdot a
$$

Les sphères $A_{0}, A_{i}, A_{\infty}$ et les composantes $N^{0}, N^{i}$ étant invariantes pendant la transformation (2.16), on trouve la loi suivante de transformation des $A_{\dot{0}}, A_{\alpha}, A_{\dot{n}}$, et $A_{\infty}$,

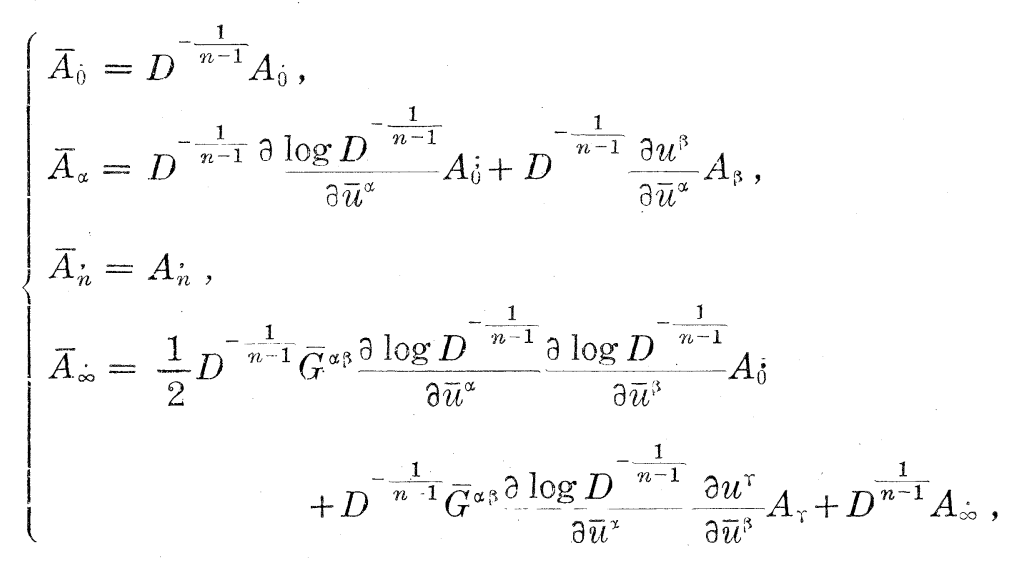

où

$$
\bar{G}^{\alpha_{\beta} \beta} \bar{G}_{\beta \gamma}=\delta_{\gamma}^{\alpha}, \quad \bar{G}_{\alpha_{\beta} \beta}=D^{-\frac{2}{n-1}} \frac{\partial u^{\gamma}}{\partial \bar{u}^{\alpha}} \frac{\partial u^{\delta}}{\partial \bar{u}^{\beta}} G_{\gamma \delta},
$$

$$
\bar{G}^{\alpha \beta}=D^{\bar{n}^{2}-1} \frac{\partial \bar{u}^{\alpha}}{\partial u^{\gamma}} \frac{\partial \bar{u}^{\beta}}{\partial u^{\delta}} G^{\gamma \delta} .
$$

On voit que les équations (2.19) ont la même forme que (2.13). 
Jusqu'à présent, nous avons supposé que la connexion soit générale, et nous avons exclusivement employé le repère naturel. Nous allons maintenant supposer que la connexion soit normale et prenons un repère de M. O. Veblen. Le fait que la connexion conforme de l'espace ambiant est normale ne donne pas toujours la conclusion que la connexion conforme sur l'hypersurface est normale, donc on ne peut pas toujours choisir un repère de M. O. Veblen sur l'hypersurface. Mais nous allons, dans la suite, essayer de chercher un repère analogue à celui de M. O. Veblen sur l'hypersurface.

Le repère de M. O. Veblen étant donné par $A_{0}, A_{i}$ et $A_{\infty}$, les composantes de la connexion satisfont aux relations

$$
\left\{\begin{array}{l}
I I_{0 i}^{0}=I I_{\infty i}^{\infty}=I I_{\propto i}^{0}=I I_{0 i}^{\infty}=0, \quad I_{0 j}^{i}=\delta_{j}^{i}, \\
I_{j k}^{0}=-\frac{R_{j k}}{n-2}+\frac{g_{j k} R}{2(n-1)(n-2)}, \\
I_{j k}^{i}=\left\{\begin{array}{c}
i \\
j k
\end{array}\right\}=\frac{1}{2} g^{i a}\left(g_{a j, k}+g_{a l, j}-g_{j k, a}\right), \\
I_{j k}^{\infty}=g_{j k}, \\
I_{\infty k k}^{i}=-\frac{R_{k}^{i}}{n-2}+\frac{\delta_{k}^{i} R}{2(n-1)(n-2)} .
\end{array}\right.
$$

Le repère de M. O. Veblen se transformant, pendant une transformation de coordonnées, d'après

$$
\left\{\begin{array}{l}
\bar{A}_{0}=A_{0}, \\
\bar{A}_{i}=\frac{\partial u^{j}}{\partial \bar{u}^{i}} A_{j}, \\
\bar{A}_{\infty}=A_{\infty},
\end{array}\right.
$$

les composantes de la connexion se transforment suivant les formules:

$$
\left\{\begin{array}{l}
\bar{I}_{0 i}^{0}=\bar{I}_{\propto i}^{\infty}=\bar{I}_{\propto i}^{0}=\bar{I}_{0 i}^{\infty}=0, \quad \bar{I}_{u j}^{i}=\delta_{j}^{i}, \\
\bar{I}_{j k}^{0}=\frac{\partial u^{a}}{\partial \bar{u}^{j}} \frac{\partial u^{b}}{\partial \bar{u}^{k}} I_{a b}^{0}, \\
\bar{I}_{j k}^{i}=\frac{\partial \bar{u}^{i}}{\partial u^{a}}\left(\frac{\partial u^{b}}{\partial \bar{u}^{j}} \frac{\partial u^{c}}{\partial \bar{u}^{k}} I_{b c}^{a}+\frac{\partial^{2} u^{a}}{\partial \bar{u}^{j} \partial \bar{u}^{k}}\right), \\
\bar{I}_{j k}^{\infty}=\frac{\partial u^{a}}{\partial \bar{u}^{j}} \frac{\partial u^{b}}{\partial \bar{u}^{k}} I I_{a b}^{\infty}, \\
\bar{I}_{\propto k}^{i}=\frac{\partial \bar{u}^{i}}{\partial u^{a}} \frac{\partial u^{b}}{\partial \bar{u}^{k}} I_{\propto b}^{a} .
\end{array}\right.
$$


Quand on multiplie $A_{0}$ par une fonction arbitraire $\lambda$ et on pose

$$
\bar{A}_{0}=\lambda A_{0},
$$

la relation

$$
d \bar{A}_{0}=d u^{i} \bar{A}_{i},
$$

nous donne

$$
\bar{A}_{i}=\lambda\left(\lambda_{i} A_{0}+A_{i}\right),
$$

où

$$
\lambda_{i}=\frac{\partial \log \lambda}{\partial u^{i}} .
$$

En remarquant que

$$
\bar{A}_{0} \bar{A}_{\infty}=-1, \quad \bar{A}_{i} \bar{A}_{\infty}=0, \quad \bar{A}_{\infty} \bar{A}_{\infty}=0,
$$

l'on trouve

$$
\bar{A}_{\infty}=\frac{1}{\lambda}\left(\frac{1}{2} g^{a b} \lambda_{a} \lambda_{b} A_{0}+g^{a b} \lambda_{a} A_{b}+A_{\infty}\right) .
$$

Pendant une transformation de repère de M. O. Veblen

$$
\left\{\begin{array}{l}
\bar{A}_{0}=\lambda A_{0}, \\
\bar{A}_{i}=\lambda\left(\lambda_{i} A_{0}+A_{i}\right), \\
\bar{A}_{\infty}=\frac{1}{\lambda}\left(\frac{1}{2} g^{a b} \lambda_{a} \lambda_{b} A_{0}+g^{a b} \lambda_{a} A_{b}+A_{\infty}\right),
\end{array}\right.
$$

les composantes de la connexion se transforment donc suivant les formules

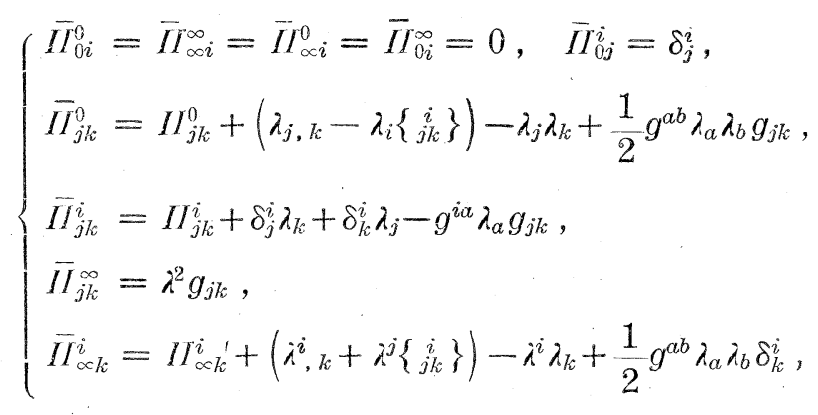

où

$$
\lambda^{i}=g^{i a} \lambda_{a} .
$$


Cela étant dans chaque espace conforme attaché à un point sur l'hypersurface, nous définissons les sphères $A_{0}, A_{\alpha}, A_{\dot{n}}$ et $A_{\infty}$ par les équations

$$
\left\{\begin{array}{l}
A_{\dot{0}}=A_{0} \\
A_{\alpha}=\frac{\partial u^{i}}{\partial u^{\alpha}} A_{i} \\
A_{\dot{n}}=B^{0} A_{0}+B^{i} A_{i}, \\
A_{\infty}=\frac{1}{2}\left(B^{0}\right)^{2}+B^{0} B^{i} A_{i}+A_{\infty},
\end{array}\right.
$$

où $B^{i}$ est un vecteur contrevariant défini sur l'hypersurface par

$$
g_{j k} \frac{\partial u^{j}}{\partial u^{\alpha}} B^{k}=0, \quad \text { et } \quad g_{j k} B^{j} B^{k}=1,
$$

et $B^{0}$ est une fonction arbitraire de $\dot{u}^{\alpha}$. Alors, il sera facilement vérifié que les $A_{\dot{0}}, A_{\alpha}, A_{\dot{n}}$ et $A_{\infty}$ satisfont aux équations suivantes

$$
\begin{aligned}
& A_{0} A_{\dot{0}}=0, \quad A_{0} A_{\alpha}=0, \quad A_{0} A_{\dot{0}}=0, \quad A_{\dot{0}} A_{\dot{\infty}}=-1, \\
& A_{\alpha} A_{\beta}=g_{\alpha \beta}, \quad A_{\alpha} A_{\dot{n}}=0, \quad A_{\alpha} A_{\infty}=0, \\
& A_{\dot{n}} A_{\dot{n}}=1, \quad A_{n} A_{\infty}=0, \\
& A_{\dot{x}} A_{\infty}=0 \text {, }
\end{aligned}
$$

où

$$
g_{\alpha,}=\frac{\partial u^{j}}{\partial u^{\alpha}} \frac{\partial u^{k}}{\partial u^{\beta}} g_{j k}
$$

et on voit bien que $A_{0}$ est un point coîncidant avec $A_{0}, A_{\alpha}$ une sphère passant $A_{\dot{0}}$ et orthogonale à la courbe paramétrique $u^{\alpha}=$ constante, $A_{\dot{n}}$ une sphère passant par $A_{\dot{0}}$ et orthogonale à toutes les sphères $A_{\alpha}$ et finalement $A_{\dot{\infty}}$ le point d'intersection autre que $A_{\dot{0}}$ de $A_{\alpha}$ et $A_{\dot{n}}$.

Quand on effectue une transformation de système de coordonnées de l'espace ambiant, les $A_{0}, A_{\alpha}$ et $A_{\infty}$ restent invariants, la fonction $B^{0}$ étant invariante pendant cette transformation.

Si l'on effectue une transformation de système de coordonnées sur l'hypersurface

$$
\bar{u}^{\alpha}=\bar{u}^{\alpha}\left(u^{\beta}\right),
$$

les $A_{0}, A_{\alpha}, A_{\dot{n}} A_{\infty}$ se transforment d'après 


$$
\left\{\begin{array}{l}
\bar{A}_{\dot{0}}=A_{0} \\
\bar{A}_{\alpha}=\frac{\partial u^{\beta}}{\partial \bar{u}^{\alpha}} A_{\beta}, \\
\bar{A}_{\dot{n}}=A_{n} \\
\bar{A}_{\infty}=A_{\infty}
\end{array}\right.
$$

parce que les fonctions $B^{0}$ et $B^{i}$ restent invariantes pendant cette transformation. Pendant la transformation (2.27) du repère de M. O. Veblen de l'espace ambiant, les $A_{\dot{0}}, A_{\alpha}, A_{\dot{n}}$ et $A_{\dot{\infty}}$. se transforment d'après

$$
\left\{\begin{array}{l}
\bar{A}_{\dot{0}}=\lambda A_{\dot{j}}, \\
\bar{A}_{\alpha}=\lambda\left(\lambda_{\alpha} A_{\dot{0}}+A_{\alpha}\right), \\
\bar{A}_{\dot{n}}=A_{\dot{n}}, \\
\bar{A}_{\dot{\infty}}=\frac{1}{\lambda}\left(\frac{1}{2} g^{\alpha_{3} \lambda_{\alpha} \lambda_{\beta}} A_{\dot{0}}+g^{\alpha \beta} \lambda_{\alpha} A_{\dot{\beta}}+A_{\dot{\infty}}\right),
\end{array}\right.
$$

où

$$
\lambda_{\alpha}=\frac{\partial \log \lambda}{\partial u^{i}} \frac{\partial u^{i}}{\partial u^{\alpha}}, \quad \bar{A}_{i}=\bar{B}_{0} \bar{A}_{0}+\bar{B}^{i} A_{i}
$$

et

$$
\bar{B}^{0}=\frac{1}{\lambda}\left(B^{0}-\lambda_{i} B^{i}\right), \quad \bar{B}^{i}=\frac{1}{\lambda} B^{i}
$$

Chapitre 3. La connexion conforme induite sur les hypersurfaces.

Nous allons, dans ce Chapitre, définir une connexion conforme sur l'hypersurface. Pour cet effet, prenons le repère naturel $\left[A_{0}, A_{i}, A_{n}\right]$ pour l'espace ambiant et définissons un repère $\left[A_{0}, A_{\alpha}, A_{\dot{r}}, A_{\dot{\alpha}}\right]$ pour l'hypersurface par

$$
\left\{\begin{array}{l}
A_{0}=a^{-\frac{1}{n-1}} A_{0}, \\
A_{\alpha}=a^{-\frac{1}{n-1}\left(\frac{\partial \log a^{-\frac{1}{n-1}}}{\partial u^{\alpha}} A_{0}+\frac{\partial u^{i}}{\partial u^{\alpha}} A_{i}\right),} \\
A_{\dot{n}}=N^{0} A_{0}+N^{i} A_{i},
\end{array}\right.
$$




$$
\begin{aligned}
A_{\dot{\infty}}=a^{\frac{1}{n-1}}\left[\frac{1}{2}\left\{\left(N^{0}\right)^{2}+a^{-\frac{2}{n-1}} G^{{ }_{\beta}} \frac{\partial \log a^{-\frac{1}{n-1}}}{\partial u^{\alpha}} \frac{\partial \log a^{-\frac{1}{n-1}}}{\partial u^{\beta}}\right\} A_{0}\right. \\
\left.+\left(N^{0} N^{i}+a^{-\frac{2}{n-1}} G^{\alpha \beta} \frac{\partial u^{i}}{\partial u^{\alpha}} \frac{\partial \log a^{-\frac{1}{n-1}}}{\partial u^{\beta}}\right) A_{i}+A_{\infty}\right] .
\end{aligned}
$$

Pour définir une connexion conforme sur l'hypersurface, nous devons d'abord déterminer la fonction arbitraire $N^{0}$. Pour cela, nous adopterons une méthode proposée par M. E. Cartan $\left({ }^{1}\right)$ et calculerons l'expression $d A_{0} d A_{n}$.

$d A_{0}$ et $d A_{i}$ étant respectivement donnés par

$$
\begin{gathered}
d A_{0}=a^{-\frac{1}{n-1}}\left(d \log a^{-\frac{1}{n-1}} A_{0}+d u^{i} A_{i}\right), \\
\text { et } d A_{n}=\left(d N^{0}+I I_{j k}^{\natural} N^{j} d u^{k}\right) A_{0}+\left(N^{0} d u^{i}+d N^{i}+I I_{h k}^{i} N^{h} d u^{k}\right) A_{i}+G_{j k} N^{j} d u^{k} A_{\infty},
\end{gathered}
$$

nous avons

$$
d A_{\dot{0}} d A_{\dot{n}}=a^{-\frac{1}{n-1}} G_{i j}\left(N^{0} \frac{\partial u^{i}}{\partial u^{\alpha}}+\frac{\partial N^{i}}{\partial u^{\alpha}}+I_{h k}^{i} N^{h} \frac{\partial u^{j}}{\partial u^{\alpha}}\right) \frac{\partial u^{j}}{\partial u^{\beta}} d u^{\alpha} d u^{\beta},
$$

en raison de la relation

$$
G_{j k} N^{j} \cdot \frac{\partial u^{k}}{\partial u^{\alpha}}=0
$$

Mais, on a d'autre part,

$$
G_{i j}\left(\frac{\partial N^{i}}{\partial u^{\alpha}}+I_{h k}^{i} N^{h} \frac{\partial u^{k}}{\partial u^{\alpha}}\right)=\frac{\partial N_{j}}{\partial u^{\alpha}}-I_{j k}^{i} N_{i} \frac{\partial u^{k}}{\partial u^{\alpha}},
$$

où

$$
N_{j}=G_{i j} N^{i}
$$

Donc, en posant

$$
\left(\frac{\partial N_{j}}{\partial u^{\alpha}}-I_{J k}^{i} N_{i} \frac{\partial u^{l k}}{\partial u^{\alpha}}\right) \frac{\partial u^{j}}{\partial u^{\beta}}=-N_{\alpha \beta}
$$

nous avons

$$
d A_{0} d A_{n}=a^{\frac{1}{n-1}}\left(N^{0} G_{\alpha \beta}-a^{-\frac{2}{n-2}} N_{\alpha_{\beta}}\right) d u^{\alpha} d u^{\beta} .
$$

(1) E. Cartan (I). 
Cela étant, nous allons choisir la fonction $N^{0}$ de manière que la moyenne de $d A_{\dot{0}} d A_{\dot{n}}$ par rapport à $d u^{\alpha}$ s'annule, soit

$$
a^{\frac{1}{n-1}}\left(N^{0} G_{\alpha_{\beta}}-a^{-\frac{2}{n-2}} N_{\alpha_{\beta}}\right) G^{\alpha_{\beta}}=0
$$

d'où

$$
N^{0}=a^{-\frac{2}{n-2}} \cdot \frac{G^{\alpha \beta} N_{\alpha \beta}}{n-1}
$$

En substituant cette valeur de $N^{0}$ dans (3.3), on trouve

$$
d A_{0} d A_{\dot{n}}=-M_{\alpha \beta} d u^{\alpha} d u^{\beta}
$$

où nous avons posé

$$
M_{\alpha \beta}=a^{-\frac{1}{n-1}}\left(N_{\alpha \beta}-\frac{G^{\gamma \delta} N_{\gamma \delta}}{n-1} G_{\alpha \beta}\right)
$$

Comme nous avons les identités

$$
\left(\frac{\partial N_{j}}{\partial u^{\alpha}}-I_{j k}^{i} N_{i} \frac{\partial u^{k}}{\partial u^{\alpha}}\right) \frac{\partial u^{j}}{\partial u^{\beta}}=-N_{i}\left(\frac{\partial^{2} u^{i}}{\partial u^{\alpha} \partial u^{\beta}}+I I_{j k}^{i} \frac{\partial u^{j}}{\partial u^{\alpha}} \frac{\partial u^{k}}{\partial u^{\beta}}\right)
$$

on voit que la quantité $N_{\alpha \beta}$ et par suite $M_{\alpha \beta}$ introduite ici est symétrique par rapport aux deux indices inférieures $\alpha$ et $\beta$.

Le tenseur $M_{\alpha \beta}$ qui satisfait à la relation

$$
G^{\alpha \beta} M_{\alpha \beta}=0
$$

va jouer, dans la suite, le rôle du second tenseur fondamental de l'hypersurface.

En substituant la valeur de $N^{0}$ donnée par (3.4) dans (3.1), nous avons

$$
\left\{\begin{array}{l}
A_{\dot{0}}=a^{-\frac{1}{n-1}} A_{0}, \\
A_{\alpha}=a^{-\frac{1}{n-1}}\left(\frac{\partial \log a^{-\frac{1}{n-1}}}{\partial u^{\alpha}} A_{0}+\frac{\partial u^{i}}{\partial u^{\alpha}} A_{i}\right), \\
A_{\dot{n}}=a^{-\frac{2}{n-1}} \frac{G^{\alpha \beta} N_{\alpha \beta}}{n-1} A_{0}+N^{i} A_{i},
\end{array}\right.
$$




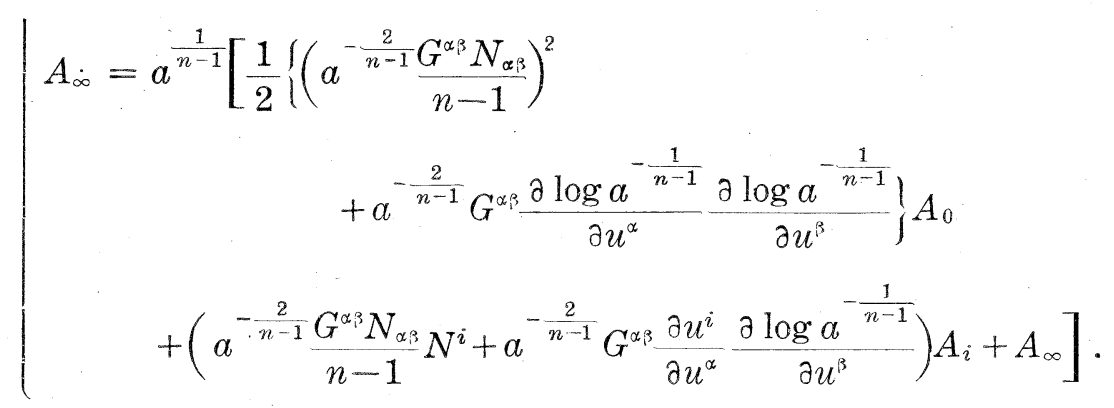

La fonction $N^{0}$ étant ainsi déterminée, nous allons calculer les composantes de la connexion par rapport au repère $\left[A_{\dot{0}}, A_{\alpha}, A_{\dot{n}}, A_{\dot{\infty}}\right]$. En tenant comptes des relations $A_{0} d A_{0}=A_{\dot{n}} d A_{0}=A_{\infty} d A_{0}=0, A_{0} d A_{\dot{n}}=$ $A_{\dot{n}} d A_{\dot{n}}=0$ et $A_{\dot{0}} d A_{\dot{\infty}}=A_{\dot{\infty}} d A_{\dot{\infty}}=0$, on aura les équations de la forme

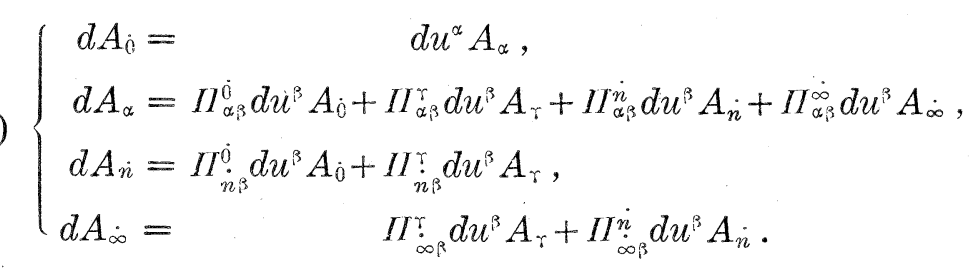

Déterminons ces composantes. On a déjà vu que le repère $\left[A_{0}, A_{\alpha}, A_{\dot{n}}, A_{\dot{\infty}}\right]$ est déterminé par les équations de la forme

$$
\left\{\begin{aligned}
A_{0} & =\rho A_{0}, \\
A_{\alpha} & =\rho\left(\rho_{\alpha} A_{0}+\frac{\partial u^{i}}{\partial u^{\alpha}} A_{i}\right), \\
A_{\dot{n}} & =N^{0} A_{0}+N^{i} A_{i}, \\
A_{\infty} & =\frac{1}{2}\left\{\frac{1}{\rho}\left(N^{0}\right)^{2}+\rho G^{\alpha \beta} \rho_{\alpha} \rho_{\beta}\right\} A_{0}+\left(\frac{1}{\rho} N^{0} N^{i}+\rho G^{\alpha \beta} \frac{\partial u^{i}}{\partial u^{\alpha}} \rho_{\beta}\right) A_{i} \\
& +\frac{1}{\rho} A_{\infty},
\end{aligned}\right.
$$

où

$$
\rho_{\alpha}=\frac{\partial \log \rho}{\partial u^{\alpha}}, \quad G_{\alpha \beta}=\rho^{2} \frac{\partial u^{j}}{\partial u^{\alpha}} \frac{\partial u^{k}}{\partial u^{\beta}} G_{j k}, \quad G^{\alpha \beta} G_{\beta \gamma}=\delta_{\Upsilon}^{\alpha} .
$$

Si $\left[A_{0}, A_{i}, A_{\infty}\right]$ forme le repère naturel et $\left[A_{\dot{0}}, A_{\alpha}, A_{\dot{n}}, A_{\dot{\infty}}\right]$ forme aussi le repère naturel, $\rho$ prend la valeur $a^{-\frac{1}{n-1}}$. 
Si l'on pose

$$
B_{\alpha^{j}}^{j}=\rho \frac{\partial u^{j}}{\partial u^{\alpha}},
$$

on obtient

$$
\left\{\begin{aligned}
G_{\alpha \beta} & =B_{\alpha}^{j} B^{k} G_{j k}, \\
0 & =B_{\alpha}^{\cdot j} N^{k} G_{j k}, \\
1 & =N^{j} N^{k} G_{j k},
\end{aligned}\right.
$$

d'où

$$
G_{j k}=B_{\cdot j}^{\alpha} B_{\cdot k}^{3} G_{\alpha \beta}+N_{j} N_{k}
$$

où nous avons posé

$$
B^{\alpha}{ }_{j}=G^{\alpha_{i}} G_{i j} B_{i}{ }^{i} .
$$

En multipliant (3.15) par $G^{i j}$ et en contractant, on trouve

$$
\delta_{k}^{i}=B_{\alpha}{ }^{i} B_{{ }_{k}}^{\alpha}+N^{i} N_{k} .
$$

En utilisant ces équations, on peut résoudre (3.11) par rapport aux $A_{0}, A_{i}$ et $A_{\infty}$,

$$
\left\{\begin{array}{l}
A_{0}=\frac{1}{\rho} A_{\dot{0}}, \\
A_{i}=-\left(\rho_{i}+\frac{1}{\rho} N^{0} N_{i}\right) A_{\dot{0}}+B_{\cdot i}^{\alpha} A_{\alpha}+N_{i} A_{\dot{n}}, \\
A_{\infty}=\frac{1}{2}\left\{\frac{1}{\rho}\left(N^{0}\right)^{2}+\rho G^{\alpha_{\beta}} \rho_{\alpha} \rho_{\dot{\beta}}\right\} A_{\dot{q}}-\rho G^{\alpha_{\beta}} \rho_{\alpha} A_{\beta}-N^{0} A_{\dot{n}}+\rho A_{\dot{\infty}},
\end{array}\right.
$$

oủ

$$
\rho_{i}=\rho_{\alpha} B_{\cdot i}^{\alpha} .
$$

Cela dit, nous avons de (3.11)

$$
\begin{aligned}
d A_{\alpha}= & \left(\rho \rho_{\alpha} \rho_{\beta}+\rho \frac{\partial \rho_{\alpha}}{\partial u^{\beta}}+\rho I_{j k}^{0} \frac{\partial u^{j}}{\partial u^{\alpha}} \frac{\partial u^{k}}{\partial u^{\beta}}\right) d u^{\beta} A_{0} \\
& +\left(B_{\beta}^{\cdot i} \rho_{\alpha}+B_{\alpha}^{i} \rho_{\beta}+\rho \frac{\partial^{2} u^{i}}{\partial u^{\alpha} \partial u^{\beta}}+\rho I_{j k}^{i} \frac{\partial u^{j}}{\partial u^{\alpha}} \frac{\partial u^{k}}{\partial u^{\beta}}\right) d u^{\beta} A_{i}+\frac{1}{\rho} G_{\alpha \beta} d u^{\beta} A_{\infty} .
\end{aligned}
$$

Donc, en substituant (3.18) dans ces équations, l'on trouve 


$$
\begin{aligned}
& d A_{\alpha}=\left[I_{j k}^{0} \frac{\partial u^{j}}{\partial u^{\alpha}} \frac{\partial u^{k}}{\partial u^{\beta}}+\left(\frac{\partial \rho_{\alpha}}{\partial u^{\beta}}-\rho \rho_{r} B_{r}^{\gamma} I_{j k}^{i} \frac{\partial u^{j}}{\partial u^{\alpha}} \frac{\partial u^{k}}{\partial u^{\beta}}\right)-\rho_{\alpha} \rho_{\beta}-\rho \rho_{r} B_{\cdot}^{\curlyvee} \frac{\partial^{2} u^{i}}{\partial u^{\alpha} \partial u^{\beta}}\right. \\
& \left.-N^{0} N_{\alpha \beta}+\frac{1}{2}\left\{\left(\frac{N^{0}}{\rho}\right)^{2}+G^{\Upsilon \delta} \rho_{\gamma} \rho_{\delta}\right\} G_{\alpha \beta}\right] d u^{\beta} A_{0} \\
& +\left[\rho B^{\Upsilon} \cdot\left(I I_{j k}^{i} \frac{\partial u^{j}}{\partial u^{\alpha}} \frac{\partial u^{k}}{\partial u^{\beta}}+\frac{\partial^{2} u^{i}}{\partial u^{\alpha} \partial u^{\beta}}\right)+\delta_{\alpha}^{\Upsilon} \rho_{\beta}+\delta_{\beta}^{\curlyvee} \rho_{\alpha}-G^{\Upsilon \delta} \rho_{\delta} G_{\alpha \beta}\right] d u^{\beta} A_{\curlyvee} \\
& +\left[\rho N_{\alpha \beta}-\frac{1}{\rho} N^{0} G_{\alpha, \beta}\right] d u^{\beta} A_{\dot{n}}+G_{\alpha \beta} d u^{\beta} A_{\infty},
\end{aligned}
$$

d'où, en comparant avec (3.10),

$$
\begin{aligned}
I_{\alpha \beta}^{0}= & I_{j k}^{0} \frac{\partial u^{j}}{\partial u^{\alpha}} \frac{\partial u^{k}}{\partial u^{\beta}}+\left(\frac{\partial \rho_{\alpha}}{\partial u^{\beta}}-\rho \rho_{\gamma} B_{r_{i}}^{\Upsilon} I_{j k}^{i} \frac{\partial u^{j}}{\partial u^{\alpha}} \frac{\partial u^{k}}{\partial u^{\beta}}\right) \\
& -\rho_{\alpha} \rho_{\beta}-\rho \rho_{\gamma} B_{\cdot}^{\curlyvee} \frac{\partial^{2} u^{i}}{\partial u^{\alpha} \partial u^{\beta}}-N^{0} N_{\alpha \beta}+\frac{1}{2}\left\{\left(\frac{N^{0}}{\rho}\right)^{2}+G^{\curlyvee} \rho_{\gamma} \rho_{\delta}\right\} G_{\alpha \beta},
\end{aligned}
$$

$$
I_{\alpha \beta}^{\Upsilon}=\rho B_{i}^{\Upsilon}\left(I_{j k}^{i} \frac{\partial u^{j}}{\partial u^{\alpha}} \frac{\partial u^{k_{k}}}{\partial u^{\beta}}+\frac{\partial^{2} u^{i}}{\partial u^{\alpha} \partial u^{\beta}}\right)+\delta_{\alpha}^{\Upsilon} \rho_{\beta}+\delta_{\beta}^{\Upsilon} \rho_{\alpha}-G^{\gamma \delta} \rho_{\delta} G_{\alpha \beta},
$$

$$
I_{\alpha \beta}^{\dot{i}}=\rho N_{\alpha \beta}-\frac{1}{\rho} N^{0} G_{\alpha \beta},
$$

(3.23) $\quad \Pi_{\alpha \beta}^{\dot{\infty}}=G_{\alpha \beta}$.

En substituant (3.21) dans (3.20), on a

$$
\begin{aligned}
I_{\alpha \beta}^{0}= & I_{j k}^{0} \frac{\partial u^{j}}{\partial u^{\alpha}} \frac{\partial u^{k}}{\partial u^{\beta}}+\left(\frac{\partial \rho_{\alpha}}{\partial u^{\beta}}-\rho_{\gamma} I_{\alpha \beta}^{\gamma}\right) \\
& +\rho_{\alpha} \rho_{\beta}-\frac{1}{2} G^{\gamma \delta} \rho_{\gamma} \rho_{\delta} G_{\alpha \beta}-N^{0} N_{\alpha_{\beta}}+\frac{1}{2}\left(\frac{N^{0}}{\rho}\right)^{2} G_{\alpha_{\beta}} .
\end{aligned}
$$

Cela étant, calculons ensuite $d A_{n}$. On a, de la troisième équation de (3.11),

$$
d A_{\dot{n}}=\left[\frac{\partial N^{0}}{\partial u^{\beta}}+I_{j k}^{0} N^{j} \frac{\partial u^{k}}{\partial u^{\beta}}\right] d u^{\beta} A_{0}+\left[N^{0} \frac{\partial u^{i}}{\partial u^{\beta}}+\frac{\partial N^{i}}{\partial u^{\beta}}+I_{j k}^{i} N^{j} \frac{\partial u^{k}}{\partial u^{\beta}}\right] d u^{\beta} A_{i} .
$$

En substituant (3.18) dans cette équation, on obtient 


$$
d A_{\dot{n}}=\left[\frac{\partial \frac{1}{\rho} N^{0}}{\partial u^{\beta}}+\frac{1}{\rho} I_{j k}^{0} N^{j} \frac{\partial u^{k}}{\partial u^{\beta}}+\rho \rho_{\alpha} N_{\beta}^{\alpha}\right] d u^{\beta} A_{\dot{\theta}}+\left(\frac{1}{\rho} N^{0} \delta_{\beta}^{\alpha}-\rho N_{\beta}\right) d u^{\beta} A_{\alpha},
$$

où

$$
N_{\beta}^{\alpha}=G^{\alpha \Upsilon} N_{\gamma_{\beta}}
$$

d'où en comparant avec (3.10)

$$
\begin{gathered}
I_{\dot{n}_{\beta}}^{\dot{0}}=\cdot \frac{\partial \frac{1}{\rho} N^{0}}{\partial u^{\beta}}+\frac{1}{\rho} I_{j k}^{0} N^{j} \frac{\partial u^{k}}{\partial u^{\beta}}+\rho \rho_{\alpha} N^{\alpha}{ }_{\beta}, \\
I_{\dot{n}_{\beta}}^{\alpha}=-\left(\rho N_{\beta}^{\alpha}-\frac{1}{\rho} N^{0} \delta_{\beta}^{\alpha}\right) .
\end{gathered}
$$

Pour calculer $I_{\infty \beta}^{r}$ et $\Pi_{\infty \beta \beta}^{\dot{n}}$ nous remarquons que

$$
A_{\alpha} d A_{\dot{\infty}}=-A_{\dot{\infty}} d A_{\alpha} \quad \text { et } \quad A_{\dot{n}} d A_{\dot{\infty}}=-A_{\dot{\infty}} d A_{\dot{n}}
$$

d'où

$$
\begin{aligned}
& I r_{\infty \beta \beta}^{\gamma}=G^{\gamma \alpha} I I_{\alpha_{\beta}}^{0}, \\
& I I_{\dot{\alpha} \beta}^{\dot{n}}=I I_{\dot{n} \beta}^{\dot{v}} .
\end{aligned}
$$

Toutes composantes dans (3.10) étant calculées, introduisons les valeurs

$$
\begin{aligned}
\rho & =a^{-\frac{1}{n-1}}, \\
\rho_{\alpha} & =\frac{\partial \log a^{-\frac{1}{n^{\alpha-1}}}}{\partial u^{\alpha}}, \\
N^{0} & =a^{-\frac{2}{n-1} \frac{G^{\gamma \delta} N_{\gamma \delta}}{n-1}}, \\
M_{\alpha \beta} & =a^{-\frac{1}{n-1}}\left(N_{\alpha \beta}-\frac{G^{\gamma \delta} N_{r \delta}}{n-1} G_{\alpha \beta}\right),
\end{aligned}
$$

dans (3.24), (3.21), (3.22), (3.23), (3.26), (3.27), (3.28) et (3.29), alors on obtiendra 
(3.30)

$$
\begin{aligned}
& I_{\alpha \beta}^{0}=I_{j k}^{o} \frac{\partial u^{j}}{\partial u^{\alpha}} \frac{\partial u^{k}}{\partial u^{\beta}}+\frac{\partial^{2} \log a^{-\frac{1}{n-1}}}{\partial u^{\alpha} \partial u^{\beta}}-\frac{\partial \log a^{-\frac{1}{n-1}}}{\partial u^{\gamma}} I_{\alpha_{\beta}}^{\gamma} \\
& +\frac{\partial \log a^{-\frac{1}{n-1}}}{\partial u^{\alpha}} \frac{\partial \log a^{-\frac{1}{n-1}}}{\partial u^{\beta}}-\frac{1}{2} G^{\gamma \delta} \frac{\partial \log a^{-\frac{1}{n-1}}}{\partial u^{\gamma}} \frac{\partial \log a^{-\frac{1}{n-1}}}{\partial u^{\delta}} G_{\alpha_{\beta}} \\
& -\left(a^{-\frac{2}{n-1}} \frac{G^{\Upsilon \delta} N_{\gamma \delta}}{n-1}\right) N_{\alpha_{\beta}}+\frac{1}{2}\left(a^{-\frac{1}{n-1}} \frac{G^{\gamma \delta} N_{\Upsilon \delta}}{n-1}\right)^{2} G_{\alpha_{\beta}}, \\
& I_{\alpha \beta}^{\curvearrowright}=a^{-\frac{1}{n-1}} B_{\cdot}^{\gamma}\left(I_{j k}^{i} \frac{\partial u^{j}}{\partial u^{\alpha}} \frac{\partial u^{k}}{\partial u^{\beta}}+\frac{\partial^{2} u^{i}}{\partial u^{\alpha} \partial u^{i}}\right) \\
& +\delta_{\alpha}^{\gamma} \frac{\partial \log a^{-\frac{1}{n-1}}}{\partial u^{\beta}}+\delta_{\beta}^{\gamma} \frac{\partial \log a^{-\frac{1}{n-1}}}{\partial u^{\alpha}}-G^{\gamma \delta} \frac{\partial \log a^{-\frac{1}{n-1}}}{\partial u^{\delta}} G_{\alpha_{\beta}}, \\
& =\frac{1}{2} G^{r \delta}\left(\frac{\partial G_{\delta \alpha}}{\partial u^{\beta}}+\frac{\partial G_{\delta \beta}}{\partial u^{\alpha}}-\frac{\partial G_{\alpha \beta}}{\partial u^{\delta}}\right), \\
& I_{\alpha^{3}}^{\dot{n}}=M_{\alpha \beta}, \\
& I_{\alpha \beta}^{\dot{\infty}}=G_{\alpha \beta}, \\
& I I_{n \beta}^{0}=\frac{1}{n-1} \frac{\partial a^{-\frac{1}{n-1}} G^{\gamma \delta} N_{\gamma \delta}}{\partial u^{\beta}}+a^{\frac{1}{n-1}} I_{j k}^{0} N^{j} \frac{\partial u^{k}}{\partial u^{\beta}} \\
& +a^{-\frac{1}{n-1} \partial \log a^{-\frac{1}{n-1}}} N_{\beta}^{\alpha} \\
& \Pi_{n_{\beta} \beta}^{\alpha}=-M_{\beta}^{\alpha}, \\
& I T_{\infty \beta}=G^{\gamma \alpha} I_{\alpha \beta}^{0} \\
& I I_{\infty \beta}^{n}=I I_{n \beta}^{0},
\end{aligned}
$$

où nous avons posé

$$
M_{\beta}^{\alpha}=G^{\alpha \delta} M_{\delta_{\beta}}
$$

Cela étant, pour induire une connexion conforme sur l'hypersurface, nous prendrons la sphère $A_{i}$ comme l'espace conforme attaché à chaque point de l'hypersurface. Si l'on prend deux points $P\left(u^{\alpha}\right)$ et $P^{\prime}\left(u^{\alpha}+d u^{\alpha}\right)$ et deux sphéres $A_{\dot{n}}$ et $A_{{ }_{n}}^{\prime}$ qui sont y attachées respectivement, $A^{\prime}{ }_{n}$ 
correspond, d'après la connexion conforme de l'espace ambiant, à la sphère $A_{\dot{r}}+d A_{\dot{n}}$. Donc, pour induire une connexion conforme sur l'hypersurface, il faut établir une correspondance conforme entre $A_{n}$. et $A_{\dot{n}}+d A_{\dot{n}}$. Pour cela, il nous sera utile une considération générale suivante:

Soient $A$ et $B$ deux sphères unitaires à $(n-1)$ dimensions dans un espace à $n$ dimensions. Pour établir une correspondance conforme entre une sphère à $(n-2)$ dimensions sur $A$ et une autre sphère à $(n-2)$ dimensions sur $B$, nous considèrerons une sphère à $(n-1)$ dimensions $X$ qui coupe $A$ orthogonalement et une autre sphère à $(n-1)$ dimensions $Y$ qui coupe $B$ aussi orthogonalement.

Si les sphères $X$ et $Y$ font toujours l'angle égal avec toutes les sphères $C$ qui coupent $A$ et $B$ orthogonalement, la sphère à $(n-2)$ dimensions qui est l'intersection de $A$ et de $X$ et la sphère à $(n-2)$ dimensions qui est l'intersection de $B$ et de $Y$ se correspondent par définition.

Nous exprimerons analytiquement ce fait comme il suit:

Deux sphères $X$ et $Y$ satisfaisant à la condition $A \cdot X=0, B \cdot Y=0$, si l'on a

$$
A \cdot C=0, \quad B \cdot C=0,
$$

on doit avoir toujours

$$
\frac{(C \cdot X)^{2}}{(C \cdot C)(X \cdot X)}=\frac{(C \cdot Y)^{2}}{(C \cdot C)(Y \cdot Y)}
$$

Donc, si l'on a $C \cdot X=0, C \cdot Y=0$ sera toujours satisfaite, et l'on voit bien que la sphère $Y$ doit avoir la forme suivante:

$$
Y=x \cdot X+a \cdot A+b \cdot B \text {. }
$$

En substituant cette équation dans (3.33), nons trouvons

$$
\frac{(C \cdot X)^{2}}{(X \cdot X)}=\frac{x^{2}(C \cdot X)^{2}}{x^{2}(X \cdot X)+a^{2}+b^{2}+2 a b(A \cdot B)^{\prime}+2 b x(B \cdot X)}
$$

grâce aux relations

$$
A \cdot A=B \cdot B=1, \quad A \cdot X=0,
$$

d'où l'on obtient

$$
a^{2}+b^{2}+2 a b(A \cdot B)+2 b x(B \cdot X)=0 .
$$

D'autre part, on a, en multipliant (3.34) par $B$, 


$$
x(B \cdot X)+a(A \cdot B)+b=0 .
$$

Les équations (3.35) et (3.36) nous donnent

$$
\begin{gathered}
a= \pm b, \\
x=-\frac{A \cdot B \pm 1}{B \cdot X} a .
\end{gathered}
$$

En choisissant le signe de $B$ de manière qu'on ait

$$
a=b \text {, }
$$

on a, de (3.34) et de (3.37),

$$
Y=-\frac{(A \cdot B+1) a}{B \cdot X} X+a A+a B
$$

ou, en multipliant par un facteur convenable,

$$
Y=X-\frac{B \cdot X}{A \cdot B+1}(A+B)
$$

En particulier, dans le cas où $B=A+d A$, on peut poser $Y=X+d X$. Donc, si l'on normalise $X$ de manière à avoir $X \cdot d X=0$, on obtient de (3.38)

$$
d X=-(X \cdot d A) A
$$

Cela étant, nous allons appliquer ce résultat pour notre $A_{\dot{n}}$.

En un point $\mathrm{M}\left(u^{\alpha}\right)$ sur l'hypersurface, une sphère $X$ à $(n-2)$ dimensions sur $A_{\dot{n}}$ peut être représentée par

$$
X=X^{\dot{0}} A_{\dot{0}}+X^{\alpha} A_{\alpha}+X^{\dot{\infty}} A_{\dot{\infty}},
$$

elle sera aussi interprétée comme une sphère à $(n-1)$ dimensions qui coupe la sphère $A_{n}$ orthogonalement. Par conséquent, la sphère correspondante $X+d X$ à $(n-2)$ dimensions sur $A_{\dot{n}}+d A_{n}$. sera facilement obtenue de la manière suivante:

$$
\begin{aligned}
d X & =-\left(X d A_{\dot{n}}\right) A_{\dot{n}} \\
& =-\left[X^{\dot{j}}\left(A_{\dot{0}} d A_{\dot{n}}\right)+X^{\alpha}\left(A_{\alpha} \cdot d A_{\dot{n}}\right)+X^{\dot{\infty}}\left(A_{\dot{\infty}} d A_{\dot{n}}\right)\right] A_{\dot{n}}
\end{aligned}
$$

donc

$$
\begin{aligned}
X+d X=X^{0}\left[A_{\dot{0}}-\left(A_{\dot{0}} \cdot d A_{\dot{n}}\right) A_{\dot{n}}\right] & +X^{\alpha}\left[A_{\alpha}-\left(A_{\alpha} \cdot d A_{\dot{n}}\right) A_{\dot{n}}\right] \\
& +X^{\dot{\circ}}\left[A_{\dot{\infty}}-\left(A_{\infty} d A_{\dot{n}}\right) A_{\dot{\dot{n}}}\right]
\end{aligned}
$$


Pour obtenir la sphère correspondante en $M^{\prime}\left(u^{\alpha}+d u^{\alpha}\right)$ soit en $A_{0}+d A_{0}$, on n'a qu'à substituer $A_{\dot{c}}, A_{\alpha}, A_{\dot{n}}$ et $A_{\dot{\infty}} \operatorname{par} A_{\dot{0}}-d A_{\dot{0}}, A_{\alpha}-d A_{\alpha}, A_{\dot{n}}-d A_{\dot{n}}$ et $A_{\dot{\infty}}-d A_{\dot{\circ}}$ respectivement dans (3.41).

$$
\begin{aligned}
X+d X=X^{\dot{0}}\left[A_{0}-d A_{0}-\left(A_{\dot{0}} d A_{\dot{n}}\right) A_{\dot{n}}\right] & +X^{\alpha}\left[A_{\alpha}-d A_{\alpha}-\left(A_{\alpha} \cdot d A_{\dot{n}}\right) A_{\dot{n}}\right] \\
& +X^{\dot{\infty}}\left[A_{\dot{\infty}}-d A_{\dot{\infty}}-\left(A_{\dot{\infty}} d A_{\dot{n}}\right) A_{\dot{n}}\right] \\
=X^{j}\left(A_{\dot{0}}-\delta A_{0}\right)+X^{\alpha}\left(A_{\alpha}-\delta A_{\alpha}\right) & +X^{\dot{\infty}}\left(A_{\dot{\infty}}-\delta A_{\dot{\infty}}\right)
\end{aligned}
$$

où nous avons posé

$$
\left\{\begin{array}{l}
\delta A_{0}=d A_{\dot{0}}+\left(A_{\dot{0}} d A_{\dot{n}}\right)=d A_{\dot{0}}-\left(d A_{\dot{0}} \cdot A_{\dot{n}}\right) \cdot A_{\dot{n}}, \\
\delta A_{\alpha}=d A_{\alpha}+\left(A_{\alpha} d A_{\dot{n}}\right)=d A_{\alpha}-\left(d A_{\alpha} \cdot A_{\dot{n}}\right) \cdot A_{\dot{n}}, \\
\delta A_{\dot{\infty}}=d A_{\dot{\infty}}+\left(A_{\dot{\alpha}} d A_{\dot{n}}\right)=d A_{\dot{\infty}}-\left(d A_{\dot{\infty}} \cdot A_{\dot{n}}\right) \cdot A_{\dot{n}} .
\end{array}\right.
$$

Les équations (3.10) et (3.42) nous donnent

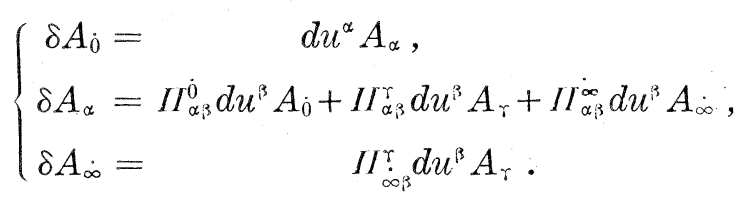

On peut aussi interpréter ce fait comme il suit: les sephères $A_{0}, A_{\alpha}$ et $A_{\dot{\infty}}$ en $M^{\prime}\left(u^{\alpha}+d u^{\alpha}\right)$ correspondent respectivement aux sphères $A_{0}+\delta A_{0}$, $A_{\alpha}+\delta A_{\alpha}$ et $A_{\dot{\infty}}+\delta A_{\dot{\infty}}$ en $M\left(u^{\alpha}\right)$ par (3.43), donc les équations (3.43) définissent une connexion conforme induite sur l'hypersurface. En général, si la sphère $X$ correspond, d'après la connexion conforme de l'espace ambiant, à la sphère $X+d X$ et que $X \cdot A_{\dot{n}}=0, X$ correspond à la sphère $X+\delta X$ d'après la connexion conforme induite sur l'hypersurface où

$$
\delta X=d X-\left(d X \cdot A_{\dot{n}}\right) A_{\dot{n}} .
$$

Nous avons pu ainsi déterminé une connexion conforme induite sur l'hypersurface, en employant le repère naturel pour tous les deux espaces ambiant et plongé.

Or, en supposant que la connexion soit normale, prenons le repère de M. O. Veblen pour l'espace ambiant et un repère analogue à celui de M. O. Veblen pour l'hypersurface plongée et considérons la connexion conforme induite.

$\left[A_{0}, A_{i}, A_{\infty}\right]$ étant le repère de M. O. Veblen, on définit un repère $\left[A_{\dot{0}}, A_{\propto}, A_{\dot{n}}, A_{\dot{\infty}}\right]$ pour l'hypersurface par les équations 


$$
\left\{\begin{array}{l}
A_{0}=A_{0} \\
A_{\alpha}=B_{\alpha}^{i} A_{i} \\
A_{\dot{n}}=B^{0} A_{0}+B^{i} A_{i} \\
A_{\infty}=\frac{1}{2}\left(B^{\dagger}\right)^{2} A_{0}+B^{0} B^{i} A_{i}+A_{\infty}
\end{array}\right.
$$

où

$$
B_{\alpha}^{i}=\frac{\partial u^{i}}{\partial u^{\alpha}}
$$

et

$$
\left\{\begin{array}{c}
g_{i j} B_{\alpha}^{i} B_{\beta}^{j}=g_{\alpha \beta}, \\
g_{i j} B_{\alpha}^{i} B^{j}=0, \\
g_{i j} B^{i} B^{j}=1 .
\end{array}\right.
$$

Pour déterminer la fonction indéterminée $E^{0}$, nous imposons, comme auparavant, la condition suivante:

$$
M_{\alpha_{\beta}} g^{\alpha_{\beta}}=0 \text {, }
$$

où

$$
d A_{j} d A_{i}=-M_{\alpha \beta} d u^{\alpha} d u^{\beta} .
$$

Or nous avons

$$
d A_{\dot{0}} \cdot d A_{\dot{n}}=g_{i j} B_{\alpha}^{i}\left(B^{0} B_{\beta}^{\cdot j}+\frac{\partial B^{j}}{\partial u^{\beta}}+\{\underset{k h}{j}\} B^{k} B_{\beta}{ }^{h}\right) d u^{\alpha} d u^{\beta}
$$

en tenant compte de

$$
d A_{0}=d u^{\alpha} B_{\alpha}^{i} A_{i},
$$

$$
\begin{aligned}
d A_{\dot{n}}= & \left(d B^{0}+I_{j k}^{0} B^{j} d u^{k}\right) A_{0} \\
& +\left(B^{0} d u^{i}+d B^{i}+\left\{\begin{array}{c}
i \\
j k
\end{array}\right\} B^{j} d u^{k}\right) A_{i}+g_{j k} B^{j} d u^{k} A_{\infty} .
\end{aligned}
$$

Donc, on trouve que

$$
M_{\alpha \beta}=-g_{i j} B_{\alpha}^{i}\left(B^{0} B_{\beta}^{j}+\frac{\partial B^{j}}{\partial u^{\beta}}+\left\{\begin{array}{c}
j \\
k h
\end{array}\right\} B^{k} B_{\beta}^{h}\right) .
$$


Mais, on a, d'autre part,

$$
-g_{i j} B_{\alpha}^{\cdot i}\left(\frac{\partial B^{j}}{\partial u^{\beta}}+\left\{\begin{array}{c}
j \\
k h h
\end{array}\right\} B^{k} B_{\beta}^{\cdot h}\right)=g_{i j} H_{\alpha \beta}{ }^{i} B^{j}:
$$

où

$$
H_{\alpha \beta}^{i}=\frac{\partial^{2} u^{i}}{\partial u^{\alpha} \partial u^{\beta}}+B_{\alpha}^{j}\{\underset{j k}{i}\} B_{\beta}^{k}-B_{\gamma}{ }^{i}\{\underset{\alpha \beta}{\gamma}\}
$$

donc, (3.53) nous donne

$$
M_{\alpha \beta}=-g_{\alpha \beta} B^{0}+H_{\alpha \beta}{ }^{i} B_{i},
$$

où

$$
B_{i}=g_{i j} B^{j}
$$

Les équations (3.48) et (3.56) nous permettent de déterminer la fonction $B^{0}$, soit

$$
\left(-g_{\alpha \beta} B^{0}+H_{\alpha \beta}{ }^{i} B_{i}\right) g^{\alpha_{\beta}^{3}}=0
$$

d'où

$$
B^{0}=\frac{1}{n-1} g^{\alpha \beta} H_{\alpha_{i}}{ }^{i} B_{i}
$$

En substituant la valeur de $B^{0}$ dans (3.56), on trouve

$$
M_{\alpha \beta}=M_{\alpha \beta}{ }^{i} B_{i},
$$

où

$$
M_{\alpha_{\beta}}^{\dot{\beta}^{i}}=H_{\alpha_{\beta}}^{i}-\frac{1}{n-1} g^{\curlyvee \delta} H_{\dot{\gamma}^{i}}^{i} g_{\alpha \beta} \cdot\left(^{1}\right)
$$

Le tenseur $M_{\alpha_{i}^{i}}^{i}$ ainsi défini est un tenseur conforme et satisfait aux relations

$$
g^{\alpha \beta} M_{\alpha \beta}^{i}=0, \quad g_{i j} B_{\alpha}^{i} M_{\beta \dot{\gamma}}^{j}=0 .
$$

Le repère $\left[A_{0}, A_{\alpha}, A_{\dot{n}}, A_{\infty}\right]$ étant ainsi complètement déterminé, nous allons ensuite déterminer les composantes de la connexion conforme par rapport à ce repère.

(1) K. Yano (3) (4) (5). 
Le repère $\left[A_{\dot{0}}, A_{\alpha}, A_{\dot{n}}, A_{\dot{\infty}}\right]$ satisfaisant aux relations

$$
\begin{aligned}
& A_{i} A_{\dot{0}}=A_{\dot{0}} A_{\alpha}=A_{\dot{0}} A_{\dot{n}}=0, \quad A_{\dot{0}} A_{\dot{\infty}}=-1, \\
& A_{\alpha} A_{\beta}=g_{\alpha \beta}, \quad A_{\alpha} A_{n}=A_{\alpha} A_{\infty}=0, \\
& A_{\dot{n}} A_{\dot{n}}=1, \quad A_{\dot{n}} A_{\dot{\infty}}=0, \\
& A_{\dot{\infty}} A_{\dot{\infty}}=0,
\end{aligned}
$$

nous avons les équations de la forme

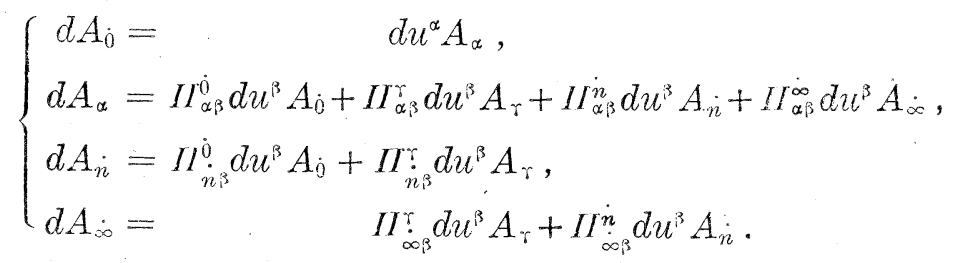

Pour déterminer les $I_{Q \beta}^{P}$ on n'a qu'à répéter le calcul analogue à celui fait au début de ce Chapitre, donc nous exposerons ici seulement les résultats:

$$
\Pi_{\alpha \beta}^{0}=I_{j k}^{0} B_{\alpha}{ }^{j} B_{\beta}{ }^{k}-\frac{1}{n-1} g^{\Upsilon \delta} H_{\curlyvee \delta} H_{\alpha \beta}+\frac{1}{2}\left(\frac{1}{n-1} g^{r \delta} H_{\Upsilon \delta}\right)^{2} g_{\alpha_{\beta}},
$$

$$
I_{\alpha_{\beta}^{\gamma}}^{\gamma}=\left\{\begin{array}{c}
r \\
\alpha_{i}^{\gamma}
\end{array}\right\}=B_{\cdot i}^{\Upsilon}\left(B_{\beta}^{j} B_{\gamma}^{k}\left\{\begin{array}{c}
i \\
j k
\end{array}\right\}+\frac{\partial^{2} u^{i}}{\partial u^{\beta} \partial u^{\gamma}}\right)
$$

(3.66) $\quad I_{\alpha_{\beta},}^{\dot{n}}=M_{\alpha_{\beta}}$,

(3.67) $\quad I_{\alpha \beta}^{\infty}=g_{\alpha \beta}$,

$$
I_{\dot{n}^{\beta}}^{\dot{0}}=\frac{\partial}{\partial u^{\beta}}\left(\frac{1}{n-1} g^{\Upsilon \delta} H_{\Upsilon \delta}\right)+I_{j k}^{0} B^{j} B_{i}^{k}
$$

(3.69) $\quad I_{n_{\beta}}^{\alpha}=-M_{\cdot \beta}^{\alpha}$,

(3.70) $\quad I_{\alpha_{\beta}^{3}}^{\Upsilon}=g^{\% \alpha} I I_{\alpha_{\beta}}^{0}$,

(3.71) $\quad I_{\dot{\infty} ; \beta}^{\dot{n}}=I I_{\dot{\infty} \beta}^{\dot{0}}$,

où nous avons posé

$$
H_{\gamma \delta}=H_{\because \delta}^{i} B_{i}, \quad B_{\cdot i}^{\Upsilon}=g_{i j} g^{r \delta} B_{\delta^{j}}^{j}, \quad M_{\cdot \beta}^{\alpha}=g^{\alpha \delta} M_{\delta \beta},
$$


Cela étant, on peut définir la connexion conforme induite sur l'hypersurface par

$$
\left\{\begin{array}{c}
\delta A_{\dot{0}}=d A_{\dot{0}}-\left(d A_{\dot{0}} A_{\dot{n}}\right) A_{n}, \\
\delta A_{\alpha}=d A_{\alpha}-\left(d A_{\alpha} A_{\dot{n}}\right) A_{\dot{n}}, \\
\dot{\delta} A_{\dot{\infty}}=d A_{\dot{\infty}}-\left(d A_{\dot{\alpha}} A_{\dot{n}}\right) A_{\dot{n}}
\end{array}\right.
$$

d'où l'on trouve

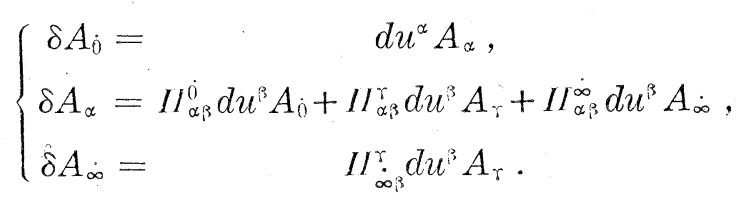

Chapitre 4. Tenseurs de courbure, les équations de Gauss et de Codazzi.

Pour traiter les tenseurs de courbure de l'hypersurface, prenons d'abord le repère naturel. La connexion conforme induite sur l'hypersurface étant donnée par

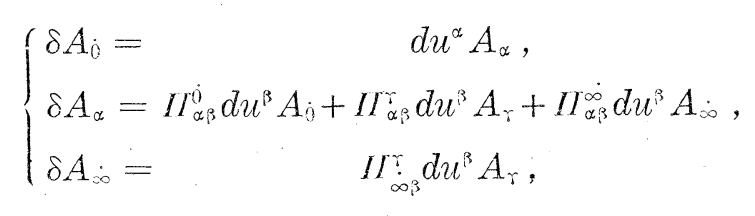

on peut définir les tenseurs de courbure de l'hypersurface comme il suit $\left({ }^{1}\right)$ :

$$
\begin{aligned}
& \Omega_{Q \gamma \delta}^{P}=I_{Q \gamma, \delta}^{P}-I_{Q \delta, r}^{P}+I_{Q r}^{R} I_{R \delta}^{P}-\Pi_{Q^{\delta}}^{R} I_{R r}^{P}, \\
& (P, Q, R, \ldots=\dot{0}, \dot{1}, \ldots n-1, \infty)
\end{aligned}
$$

c'est-à-dire

$$
\begin{aligned}
& \Omega_{\dot{0} \delta \delta}^{\dot{j}}=I I_{\gamma \delta}^{\dot{0}}-I_{\delta r}^{\dot{0}}, \\
& \Omega_{\beta \gamma \delta}^{\dot{0}}=H_{\beta \gamma, \delta}^{0}-H_{\beta \delta, \gamma}^{\dot{0}}+I_{\beta \gamma}^{\varepsilon} H_{\varepsilon \delta}^{\dot{\theta}}-I_{\beta \delta}^{\varepsilon} I_{\varepsilon \gamma}^{0}, \\
& \Omega_{\beta \gamma \delta}^{\alpha}=I_{\beta \gamma \delta}^{\alpha}+I_{\beta \gamma}^{0} \delta_{\delta}^{\alpha}-I_{\beta \delta}^{0} \delta_{r}^{\alpha}+G_{\beta \gamma} I_{\infty \delta \delta}^{\alpha}-G_{\beta \delta} I_{\dot{\alpha}}^{\alpha}, \\
& \Omega_{\dot{\infty} \delta \delta}^{\dot{0}}=-\Omega_{\dot{0} \delta}^{\dot{0}} \text {, }
\end{aligned}
$$

(1) K. Yano (2). 


$$
\begin{aligned}
& \Omega_{\text {○; } \delta}^{x}=G^{\alpha \beta} Q_{\beta \gamma \delta}^{0},
\end{aligned}
$$

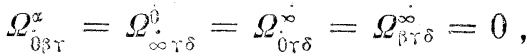

où nous avons posé

$$
\Pi_{\beta \gamma \delta}^{\alpha}=\Pi_{\beta \gamma, \delta}^{\alpha}-I_{\beta \delta, \gamma}^{\alpha}+I_{\beta \gamma}^{\varepsilon} \Pi_{\varepsilon \delta}^{\alpha}-\Pi_{\beta \delta}^{\varepsilon} \Pi_{\varepsilon \gamma}^{\alpha},
$$

la virgule désignant la dérivée partielle par rapport aux variables $u^{\delta}$.

Nous chercherons, dans ce Chapitre, les relations qu'il existe entre les tenseurs de courbure $\Omega_{Q r o}^{P}$ définis sur l'hypersurface et les tenseurs de courbure $\Omega_{B k h}^{A}(A, B, C, \cdots=0,1,2, \cdots, n, \infty)$ de l'espace ambiant à connexion conforme :

$$
\begin{aligned}
& \Omega_{0 k h}^{0}=\Pi_{k h}^{c}-I_{h k}^{0}, \\
& \Omega_{j k h}^{0}=\Pi_{j k, h}^{0}-\Pi_{j h, k}^{0}+\Pi_{j k}^{a} \Pi_{a h}^{0}-\Pi_{j h}^{a} \Pi_{a k}^{0}, \\
& \Omega_{j k h}^{i}=\Pi_{j k h}^{i}+\Pi_{j k}^{0} \delta_{h}^{i}-I_{j h}^{0} \delta_{k}^{i}+G_{j k} \Pi_{\infty h}^{i}-G_{j h} \Pi_{\infty k}^{i}, \\
& \Omega_{\infty k h}^{\infty}=-\Omega_{0 k h}^{0}, \\
& \Omega_{\infty k h}^{i}=G^{i j} \Omega_{j k h}^{0}, \\
& \Omega_{0 k h}^{i}=\Omega_{j k h}^{\infty}=\Omega_{\infty k h}^{0}=\Omega_{0 k h}^{\infty}=0,
\end{aligned}
$$

où

$$
\Pi_{j k h}^{i}=\Pi_{j k, h}^{i}-I_{j h, k}^{i}+\Pi_{j k}^{a} \Pi_{a h}^{i}-\Pi_{j h}^{a} \Pi_{a k}^{i},
$$

et la virgule désigne la dérivée partielle par rapport aux variables $u^{h}$.

Pour cet effet, posons (3.9) sous la forme suivante:

$$
A_{P}=B_{P}{ }^{A} A_{A} \text {. }
$$

Alors, en remarquant que

on tire de (4.17)

$$
d A_{P}=\delta A_{P}-\left(A_{P} d A_{\dot{n}}\right) A_{\dot{n}},
$$

$$
\Pi_{P \gamma}^{Q} d u^{r} A_{Q}-\left(A_{P} d A_{\dot{n}}\right) A_{\dot{n}}=B_{P}{ }^{A}, r d u^{\Upsilon} A_{A}+B_{P}^{{ }^{C}} I I_{C k}^{A} \frac{\partial u^{k}}{\partial u^{r}} d u^{\Upsilon} A_{A}
$$

Mais, on a d'autre part

$$
\begin{aligned}
\left(A_{P} d A_{\dot{r}}\right) & =A_{P}\left(I_{\dot{n} \Upsilon}^{\dot{0}} d u^{\curlyvee} A_{0}+\Pi_{\dot{n} \Upsilon}^{\varepsilon} d u^{\curlyvee} A_{\varepsilon}\right) \\
& =-M_{P_{\curlyvee}} d u^{\curlyvee}
\end{aligned}
$$


où

(4.20) $\quad M_{\dot{0} \gamma}=0$ et $M_{\dot{\infty} \gamma}=\frac{1}{n-1} \frac{\partial a^{-\frac{1}{n-1}} G^{\delta \varepsilon} N_{\delta \varepsilon}}{\partial u^{\gamma}}+a^{\frac{1}{n-1}} I_{j k}^{0} N^{j} \frac{\partial u^{k}}{\partial u^{\gamma}}$

$$
+a^{-\frac{\tau}{n-1} \partial \log a^{-\frac{1}{n-1}}} \frac{\partial u^{\alpha}}{\partial u_{r}^{\alpha}}
$$

$M_{\alpha \beta}$ ayant été défini par (3.6). Donc les équations (4.18) nous donnent $B_{Q}{ }^{A} I_{P \curlyvee}^{Q} d u^{\Upsilon} A_{A}+M_{P r} d u^{\curlyvee} N^{A} A_{A}=B_{P}{ }^{A}, r d u^{\Upsilon} A_{A}+B_{P}{ }^{C} I I_{C k}^{A} \frac{\partial u^{k}}{\partial u^{\curlyvee}} d u^{\curlyvee} A_{A}$, d'où l'on obtient

$$
B_{Q}^{A} \Pi_{P \curlyvee}^{Q}=B_{P}^{C} I_{C k}^{A} \frac{\partial u^{k}}{\partial u^{\gamma}}+B_{P, \Upsilon}^{A}-M_{P \curlyvee} N^{A}
$$

En dérivant (4.21) par rapport à $u^{\delta}$, on trouve

$$
\begin{aligned}
& B_{Q, \delta}^{A} I_{P \curlyvee}^{Q}+B_{Q}^{A} I_{P \Upsilon, \delta}^{Q}=B_{P, \delta}^{C} I_{C k}^{A} \frac{\partial u^{k}}{\partial u^{\Upsilon}}+B_{P}^{C} I_{C k, h}^{A} \frac{\partial u^{k}}{\partial u^{\gamma}} \frac{\partial u^{h}}{\partial u^{\delta}} \\
& +B_{P}{ }^{C} I_{C k}^{A} \frac{\partial^{2} u^{k}}{\partial u^{\curlyvee} \partial u^{\delta}}+B_{P, \gamma, \delta}{ }^{A}-M_{P \curlyvee, \delta} N^{A}-M_{P \curlyvee} N^{A}{ }_{, \delta} \cdot
\end{aligned}
$$

Les $B_{Q}{ }_{Q}{ }^{A}$ étant donnés par

$$
B_{Q^{\prime}, \delta}^{A}=B_{R}{ }^{A} I_{Q \delta}^{R}-B_{Q^{B}} I_{B k}^{A} \frac{\partial u^{k}}{\partial u^{\delta}}+M_{Q \delta} N^{A},
$$

les équations (4.22) deviennent

$$
\begin{gathered}
\left(B_{R}^{A} I_{Q^{\delta}}^{R}-B_{Q}^{B} I_{B k}^{A} \frac{\partial u^{k}}{\partial u^{\delta}}+M_{Q \delta} N^{A}\right) I_{P \curlyvee}^{Q}+B_{Q}^{A} I_{P \curlyvee, \delta}^{Q} \\
=\left(B_{R}^{C} I_{P \delta}^{R}-B_{P}^{B} I_{B h}^{C} \frac{\partial u^{h}}{\partial u^{\delta}}+M_{P \delta} N^{C}\right) I_{C k}^{A} \frac{\partial u^{k}}{\partial u^{\gamma}} \\
+B_{P}^{\cdot C} I I_{C k, h}^{A} \frac{\partial u^{k}}{\partial u^{\gamma}} \frac{\partial u^{h}}{\partial u^{\delta}}+B_{P}^{C} I_{C k}^{A} \frac{\partial^{2} u^{k}}{\partial u^{\gamma} \partial u^{\delta}} \\
+B_{P, \gamma, \delta}^{A}-M_{P \gamma, \delta} N^{A}-M_{P \gamma} N^{A}, \delta
\end{gathered}
$$

En permutant $\gamma$ et $\delta$ et en soustrayant les équations obtenues de (4.23), on trouve 


$$
\begin{aligned}
B_{Q}{ }^{A} Q_{P \gamma \delta}^{\rho}=B_{P}{ }^{B} \Omega_{B k h}^{A} & \frac{\partial u^{k}}{\partial u^{\gamma}} \frac{\partial u^{h}}{\partial u^{\delta}}-M_{P \gamma} N_{; \delta}^{A} \\
& +M_{P \delta} N^{A} ; \curlyvee-\left(M_{P \curlyvee ; \delta}-M_{P \delta ; \curlyvee}\right) N^{A}
\end{aligned}
$$

où nous avons posé

$$
\begin{aligned}
& N_{; \delta}^{A}=N^{A}{ }_{, \delta}+N^{B} I_{B k}^{A} \frac{\partial u^{k}}{\partial u^{\delta}}, \\
& M_{P \curlyvee ; \delta}=M_{P \curlyvee, \delta}-M_{Q \curlyvee} I_{P \delta}^{Q}-M_{P \varepsilon} I_{\curlyvee \delta}^{\varepsilon} .
\end{aligned}
$$

Cela étant, on tire de $A_{\dot{n}}=N^{A} A_{A}$,

$$
d A_{\dot{n}}=N^{A} ; \delta d u^{\delta} A_{A},
$$

d'où en tenant compte de (4.19)

$$
G_{A B} B_{P}^{B} N^{A} ; \delta=-M_{P \delta},
$$

et

$$
N_{A} N_{; \delta}^{A}=0, \quad\left(N_{A}=G_{A B} N^{B}\right)
$$

où nous posons

$$
A_{A} A_{B}=G_{A B}, \quad A_{P} A_{Q}=G_{P Q} .
$$

Donc, en multipliant (4.28) par

$$
B^{P} \cdot C=G_{B C} G^{P Q} B_{Q}{ }^{B} \quad\left(G^{P Q} G_{Q R}=\delta_{R}^{P}\right)
$$

et en sommant, I'on obtient

$$
B^{P} \cdot{ }_{C} M_{P \delta}=-G_{A C} N^{A} \cdot \delta
$$

grâce aux relations

$$
B_{P}^{B} B^{Q}{ }_{B}=\delta_{P}^{Q}, \quad B_{P}^{{ }^{B}} B^{P} \cdot C=\delta_{?}^{B}-N^{B} N_{C} .
$$

En multipliant (4.28) par $G^{Q P}$ et en sommant, on trouve

$$
B_{\cdot{ }_{A}}^{Q} N^{A}{ }_{\delta}=-G^{Q P} M_{P \delta}=-M_{{ }_{\delta}}^{Q} .
$$

Ceci dit, en multipliant (4.24) $\operatorname{par} B^{R}{ }_{A}$ et en sommant, on trouve

$$
\Omega_{P \curlyvee \delta}^{R}=B_{\cdot}^{R}{ }_{A} B_{P}^{\cdot B} \Omega_{B k h}^{A} \frac{\partial u^{k}}{\partial u^{\curlyvee}} \frac{\partial u^{h}}{\partial u^{\delta}}+M_{P \curlyvee} M^{R}{ }_{\delta}-M_{P_{\delta}} M_{\gamma}^{R},
$$


grâce à

$$
B_{. A}^{R} N^{A}=0
$$

En multipliant en suite (4.24) par $N_{A}$ et en sommant, on obtient

$$
N_{A} B_{P}^{B} \Omega_{B k h}^{A} \frac{\partial u^{k}}{\partial u^{\gamma}} \frac{\partial u^{h}}{\partial u^{\delta}}-M_{P \curlyvee ; \delta}+M_{P \delta ; \gamma}=0
$$

en raison des relations

$$
B_{Q}{ }^{A} N_{A}=0, \quad N_{A} N_{; \delta}^{A}=0 \text { et } N_{A} N^{A}=1 .
$$

En posant $A, B, \cdots=0, i, \infty ; P, \mathrm{Q}, \cdots=\dot{0}, \alpha, \dot{\infty}$, respectivement dans (4.34), on obtiendra

$$
\begin{aligned}
& \Omega_{\dot{0} \delta \delta}^{\dot{9}}=\Omega_{0 k h}^{0} \frac{\partial u^{k}}{\partial u^{\gamma}} \frac{\partial u^{h}}{\partial u^{\delta}}, \\
& \Omega_{\beta \gamma \delta}^{\dot{0}}=B_{\cdot 0}^{\dot{0}} B_{\beta}^{\cdot 0} \Omega_{0 k h}^{0} \frac{\partial u^{k}}{\partial u^{\curlyvee}} \frac{\partial u^{h}}{\partial u^{\delta}}+B_{\cdot 0}^{\dot{0}} B_{\beta}^{\cdot j} \Omega_{j k h}^{0} \frac{\partial u^{k}}{\partial u^{\gamma}} \frac{\partial u^{h}}{\partial u^{\delta}} \\
& +B_{\cdot i}^{0} B_{\beta}{ }^{j} \Omega_{j k h}^{i} \frac{\partial u^{k}}{\partial u^{\gamma}} \frac{\partial u^{h}}{\partial u^{\delta}}-M_{\beta \gamma} M_{\dot{\infty} \delta}+M_{\beta \delta} M_{\dot{\infty}},
\end{aligned}
$$

$$
\Omega_{\beta \gamma \delta}^{\alpha}=B_{\cdot i}^{\alpha} B_{\beta}^{\cdot j} S_{j k h}^{i} \frac{\partial u^{k}}{\partial u^{\gamma}} \frac{\partial u^{h}}{\partial u^{\delta}}+M_{\beta \gamma} M_{\cdot \delta}^{\alpha}-M_{\beta \delta} M_{\cdot \gamma}^{\alpha}
$$

On peut appeler (4.40) les équations de Gauss d'une hypersurface dans un espace à connexion conforme.

D'une manière analogue, on tire de (4.36)

$$
\begin{aligned}
& N_{i} B_{\beta}^{\cdot j} \Omega_{j k h}^{i} \frac{\partial u^{k}}{\partial u^{r}} \frac{\partial u^{h}}{\partial u^{\delta}}-M_{\beta r ; \delta}+M_{\beta \delta: \gamma}=0, \\
& N_{i} B_{\infty}^{j} \Omega_{j k h}^{i} \frac{\partial u^{k}}{\partial u^{\gamma}} \frac{\partial u^{h}}{\partial u^{\delta}}+N_{i} B_{\infty}^{: \infty} \Omega_{\infty k h}^{i} \frac{\partial u^{k}}{\partial u^{\gamma}} \frac{\partial u^{h}}{\partial u^{\delta}} \\
& +N_{\infty} B_{\infty}^{\dot{\infty}^{\infty}} \Omega_{\propto k h}^{\infty} \frac{\partial u^{k}}{\partial u^{\gamma}} \frac{\partial u^{h}}{\partial u^{\delta}}-M_{\dot{\infty}, \delta}+M_{\dot{\infty} ; \gamma}=0 .
\end{aligned}
$$

Les équations (4.41) peuvent être regardées comme les équations de Codazzi pour l'hypersurface, les équations (4.42) étant une conséquence de (4.41).

Cela étant, supposons ensuite que la connexion conforme de l'espace ambiant soit normale et prenons le repère de M. O. Veblen $\left[A_{0}, A_{i}, A_{\infty}\right]$ 
pour l'espace ambiant. Alors la connexion normale sera exprimée par les équations

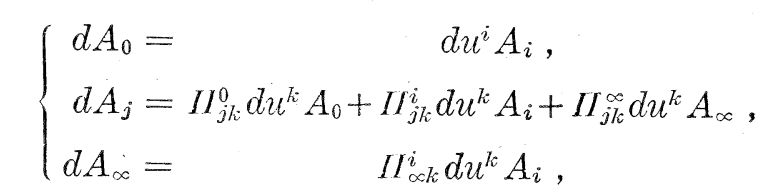

où

$$
\left\{\begin{array}{c}
I I_{j k}^{0}=-\frac{R_{j k}}{n-2}+\frac{g_{j k} R}{2(n-1)(n-2)}, \\
I_{j k}^{i}=\left\{\begin{array}{l}
i \\
j k
\end{array}\right\}, \quad I I_{j k}^{\infty}=g_{j k}, \\
I_{\propto \circ k}^{i}=-\frac{R_{k}^{i}}{n-2}+\frac{\delta_{k}^{i} R}{2(n-1)(n-2)},
\end{array}\right.
$$

et

$$
\left\{\begin{array}{l}
R_{j k}=R_{j k i}^{i}, \quad R_{k}^{i}=g^{i j} R_{j k}, \quad R=g^{j k} R_{j k}, \\
R_{j k h}^{i}=\left\{\begin{array}{c}
i \\
j k
\end{array}\right\}_{, h}-\left\{\begin{array}{c}
i \\
j h
\end{array}\right\}_{, k}+\left\{\begin{array}{c}
a \\
j k
\end{array}\right\}\left\{\begin{array}{c}
i \\
a h
\end{array}\right\}-\left\{\begin{array}{c}
a \\
j h
\end{array}\right\}\left\{\begin{array}{c}
i \\
a k
\end{array}\right\} .
\end{array}\right.
$$

En partant de ces composantes de la connexion, on peut former les tenseurs de courbure suivantes:

$$
\Omega_{B k h}^{A}=\Pi_{B h, h}^{A}-I I_{B h, k}^{A}+I I_{B k}^{C} I I_{C h}^{A}-I I_{B h}^{C} I I_{C k}^{A},
$$

c'est-à-dire

$$
\left\{\begin{aligned}
\Omega_{j k h}^{\dagger} & =-\frac{1}{n-3} C_{j k h \cdot i}^{i}, \\
\Omega_{j k h}^{i} & =C_{j k h}^{i}, \\
\Omega_{\propto k h h}^{i} & =g^{i j} \Omega_{j k h}^{0},
\end{aligned}\right.
$$

les autres étant tous nuls, où

$$
\begin{aligned}
C_{j k h}^{i}=R_{j k h}^{i}-\frac{1}{n-2}\left(R_{j k} \delta_{h}^{i}-R_{j h} \delta_{k}^{i}+g_{j k} R_{h}^{i}-g_{j h} R_{k}^{i}\right) \\
+\frac{R}{(n-1)(n-2)}\left(g_{j k} \delta_{h}^{i}-g_{j h} \delta_{k}^{i}\right)
\end{aligned}
$$

désigne le tenseur conforme de M. Weyl et / la dérivée covariante par rapport aux symboles de Christoffel $\left\{\begin{array}{c}i \\ j k\end{array}\right\}$.

Cela dit, définissons un repère $\left[A_{0}, A_{\alpha}, A_{\dot{n}}, A_{\dot{\infty}}\right]$ sur l'hypersurface par les équations 


$$
\left\{\begin{array}{l}
A_{\dot{0}}=A_{0}, \\
A_{\alpha}=B_{\alpha}^{i} A_{i}, \\
A_{\dot{n}}=B^{0} A_{0}+B^{i} A_{i}, \\
A_{\infty}=\frac{1}{2}\left(B^{0}\right)^{2} A_{0}+B^{0} B^{i} A_{i}+A_{\infty} .
\end{array}\right.
$$

La connexion induite sur l'hypersurface étant définie par

$$
\left\{\begin{array}{lcc}
\delta A_{\dot{0}}=d A_{\dot{0}}-\left(d A_{\dot{0}} A_{\dot{n}}\right) A_{\dot{n}} & = & d u^{\alpha} A_{\alpha} \\
\delta A_{\alpha}=d A_{\alpha}-\left(d A_{\alpha} A_{\dot{n}}\right) A_{\dot{n}} & =I I_{\alpha \beta}^{\dot{\alpha}} d u^{\beta} A_{0}+I I_{\alpha \beta}^{\gamma} d u^{\beta} A_{r}+I I_{\alpha \beta}^{\dot{\alpha}} d u^{\beta} A_{\dot{\infty}} \\
\delta A_{\dot{\infty}}=d A_{\dot{\alpha}}-\left(d A_{\dot{\alpha}} A_{\dot{n}}\right) A_{\dot{n}} & = & I I_{\dot{\alpha} \beta}^{\gamma} d u^{\beta} A_{\curlyvee}
\end{array}\right.
$$

on peut définir les tenseurs de courbure de cette connexion induite par

$$
\Omega_{Q \gamma \delta}^{P}=I I_{Q r, \delta}^{P}-I_{Q^{\delta}, \gamma}^{P}+I I_{Q \gamma}^{R} I_{R^{\delta}}^{P}-I_{Q \delta}^{R} I_{R \gamma}^{P},
$$

soit par

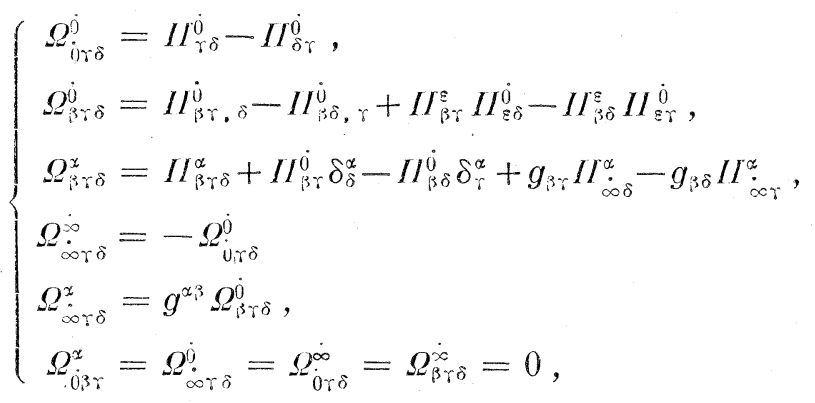

où

$$
I I_{\beta \gamma \delta}^{\alpha}=I_{\beta \gamma, \delta}^{\alpha}-I I_{\beta \delta, \gamma}^{\alpha}+I_{\beta \gamma}^{\varepsilon} I I_{\varepsilon \delta}^{\alpha}-I I_{\beta \delta}^{\varepsilon} I I_{\varepsilon \gamma}^{\alpha} .
$$

Les relations entre $I_{B k}^{A}$ et $I_{Q k}^{P}$, étant données par (3.64), (3.65), ... et (3.71), nous allons chercher les relations entre les tenseurs de courbure de l'espace ambiant et de l'hypersurface.

Le calcul étant tout à fait le même qu'auparavant, nous écrivons ici seulement les résultats :

$$
\Omega_{0 \gamma \delta}^{0}=B_{\gamma}^{\cdot k} B_{\delta}{ }^{h} \Omega_{0 k h}^{0},
$$




$$
\begin{aligned}
& \Omega_{\beta \gamma \delta}^{\dot{0}}=B_{\beta}{ }^{j} B_{\gamma^{k}}{ }^{k} B_{\dot{\delta}}{ }^{h} \Omega_{j k h}^{0}-B^{0} B_{i} B_{\beta}{ }^{j} B_{r}{ }^{k} B_{\dot{\delta}}{ }^{h} Q_{j k h}^{i} \\
& -M_{\beta \gamma}\left[\frac{1}{n-1} \frac{\partial g^{\natural \rho} H_{\varepsilon \rho}}{\partial u^{\delta}}+I_{j k}^{\hat{v}} B^{j} B_{\delta}^{k}\right] \\
& +M_{\beta \delta}\left[\frac{1}{n-1} \frac{\partial g^{q \rho} H_{\varepsilon \rho}}{\partial u^{\curlyvee}}+I_{j k}^{0} B^{j} B_{r}^{\cdot k}\right],
\end{aligned}
$$

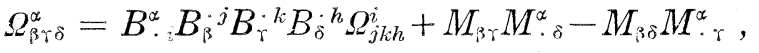

$$
\begin{aligned}
& B_{i} B_{\beta}{ }^{j} B_{\curlyvee}{ }^{k} B_{\delta}{ }^{h} \Omega_{j k h}^{i}-M_{\beta \gamma \delta}+M_{\beta \delta \gamma}=0,
\end{aligned}
$$

où

$$
M_{\beta \gamma \delta}=M_{\beta \gamma \| \delta}-g_{\beta \delta}\left[\frac{1}{n-1} \frac{\partial g^{\varepsilon p} H_{\varepsilon p}}{\partial u^{r}}+I_{j k}^{0} B^{j} B_{r}{ }^{k}\right],
$$

II désignant la derivée covariante par rapport aux symboles de Christoffel $\left\{\begin{array}{c}\alpha \\ \beta \gamma\end{array}\right\}$ formés avec les $g_{\alpha \beta}$.

Le tenseur $\Omega_{0 k h}^{0}$ étant toujours nul, on voit que $\Omega_{0 \gamma \delta}^{0}$ est identiquement nul, par conséquent, $I_{\gamma \delta}^{\dot{\theta}}$ est symétrique par rapport à $\gamma$ et $\delta$.

En contractant, dans (4.56), par rapport à $\alpha$ et $\delta$, nous avons

$$
\Omega_{\beta \gamma \alpha}^{\alpha}=B_{i}^{h} B_{\beta}{ }^{j} B_{\gamma}{ }^{k} \Omega_{j k h}^{i}-M_{\beta \alpha} M_{\cdot \gamma}^{\alpha},
$$

où

$$
B_{i}^{h}=B_{\cdot{ }_{i}}^{\alpha} B_{\alpha}{ }^{h}
$$

La connexion induite n'étant pas toujours normale, $\mathcal{L}_{\beta \gamma \alpha}^{\alpha}$ ne s'annule pas en général. En multipliant (4.58) par $g^{\text {ir }}$ et en sommant, on trouve

$$
g^{\beta \gamma} \Omega_{\beta \gamma \alpha}^{x}=B_{i}^{h} B^{j k} \Omega_{j k h}^{i}-M_{\cdot \alpha}^{\beta} M_{* \beta}^{x},
$$

où

$$
B^{j k}=B_{\beta}^{\cdot j} B_{\gamma}^{\cdot k} g^{\beta \gamma}
$$

Or, le tenseur de courbure étant défini par

$$
\Omega_{\beta \gamma \delta}^{\alpha}=R_{\beta \gamma \delta}^{\alpha}+I_{\beta \gamma}^{\dot{0}} \delta_{\delta}^{\alpha}-I_{\beta \delta}^{\dot{0}} \delta_{\gamma}^{\alpha}+g_{\beta \gamma} I_{\dot{\infty} \delta}^{\alpha}-g_{\beta \delta} I_{\dot{\alpha} \gamma}^{\alpha},
$$

où

$$
R_{\beta \gamma \delta}^{x}=\left\{\begin{array}{c}
\alpha \\
\beta \gamma
\end{array}\right\}_{\delta}-\left\{\begin{array}{c}
\alpha \\
\beta \delta
\end{array}\right\}_{, i}+\left\{\begin{array}{c}
\varepsilon \\
\beta \gamma
\end{array}\right\}\left\{\begin{array}{c}
\alpha \\
\varepsilon \delta
\end{array}\right\}-\left\{\begin{array}{c}
\varepsilon \\
\beta \delta
\end{array}\right\}\left\{\begin{array}{c}
\alpha \\
\varepsilon \delta \delta
\end{array}\right\},
$$

on en tire

$$
\Omega_{\beta \gamma \alpha}^{\alpha}=R_{\beta \gamma}+(n-3) I_{\beta \gamma}^{0}+g_{\beta \gamma} g^{q \rho} I_{\mathrm{q} \rho}^{0},
$$


et

$$
g^{\beta \curlyvee} \Omega_{\beta \curlyvee \alpha}^{\alpha}=g^{\beta \curlyvee} R_{\beta \gamma}+2(n-2) g^{\xi \rho} I_{\varepsilon \rho}^{\dot{0}} .
$$

En substituant (4.62) dans (4.61), on obtient

$$
\Omega_{\beta \gamma \alpha}^{\alpha}-\frac{g_{\beta \gamma} g^{q \rho} \Omega_{\varepsilon \rho \alpha}^{\alpha}}{2(n-2)}=R_{\beta \gamma}-\frac{g_{\beta \gamma} g^{q \rho} R_{\varepsilon \rho}}{2(n-2)}+(n-3) I_{\beta \uparrow}^{0}
$$

d'où

$$
\left[\frac{\Omega_{\beta \gamma \alpha}^{\alpha}}{n-3}-\frac{g_{\beta \gamma} g^{\varepsilon p} \Omega_{\varepsilon p \alpha}^{\alpha}}{2(n-2)(n-3)}\right]-\left[\frac{R_{\beta \gamma}}{n-3}-\frac{g_{\beta \gamma} g^{\varepsilon p} R_{\varepsilon p}}{2(n-2)(n-3)}\right]=\Pi_{\beta \gamma}^{0},
$$

et par conséquent

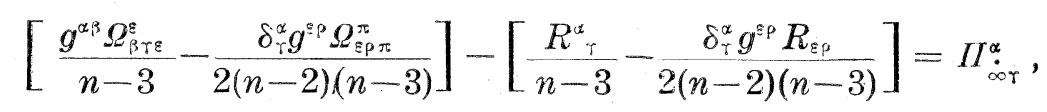

où

$$
R_{\gamma}^{\alpha}=g^{\alpha_{3}} R_{\beta \gamma}
$$

En substituant (4.63) et (4.64) dans (4.60), on trouve finalement

$$
\begin{aligned}
& \Omega_{\beta \gamma \delta}^{\alpha}-\frac{1}{n-3}\left[\Omega_{\beta \curlyvee \varepsilon}^{\varepsilon} \delta_{\delta}^{\alpha}-\Omega_{\beta \delta \varepsilon}^{\varepsilon} \delta_{\curlyvee}^{\alpha}+g_{\beta \gamma} g^{\alpha \Sigma} \Omega_{\varepsilon \delta p}^{p}-g_{\beta \delta} g^{\alpha} \Omega_{\varepsilon \gamma p}^{\rho}\right] \\
& +-\frac{g^{\xi \rho} \Omega_{\varepsilon p \pi}^{\pi}}{(n-2)(n-3)}\left(g_{0} \delta_{\delta}^{\gamma}-g_{\beta \delta} \delta_{\%}^{\alpha}\right) \\
& =R_{\beta \gamma \delta}^{x}-\frac{1}{n-3}\left[R_{\beta \gamma} \delta_{\delta}^{x}-R_{\beta \delta} \delta_{\gamma}^{x}+g_{\beta \gamma} R_{. \delta}^{\alpha}-g_{\beta \delta} R_{\cdot \gamma}^{\alpha}\right] \\
& +\frac{g^{\varepsilon \rho} R_{\xi \rho}}{(n-2)(n-3)}\left(g_{\beta \gamma} \delta_{\delta}^{\gamma},-g_{\beta \delta} \delta_{\gamma}^{\gamma}\right) .
\end{aligned}
$$

Cela étant, désignons par $C_{j k h}^{i}$ et $C_{\beta \gamma \delta}^{\alpha}$ les tenseurs de courbure de Weyl de l'espace ambiant et de l'hypersurface respectivement. Alors, en substituant les équations (4.58) et (4.59) dans (4.65), on trouve

$$
\begin{aligned}
& \mathcal{Q}_{\beta \curlyvee \delta}^{\alpha}=C_{\beta \gamma \delta}^{\alpha}+\frac{1}{n-3}\left[B_{i}^{h} B_{i \zeta \gamma}^{j k} C_{j k h}^{i} \delta_{\delta}^{\alpha}-B_{i}^{h} B_{i j \delta}^{j k} C_{j k h}^{i} \delta_{\curlyvee}^{x}+\right. \\
& \left.+g_{\beta \curlyvee} g^{\alpha \varepsilon} B_{i}^{h} B_{\Sigma \delta}^{j k} C_{j k h}^{i}-g_{\beta \delta} g^{\alpha \varepsilon} B_{i}^{h} B_{\varepsilon \gamma}^{j k} C_{j k l h}^{i}\right] \\
& -\frac{B_{i}^{h} B^{j k} C_{j k h}^{i}}{(n-2)(n-3)}\left(g_{\beta \gamma} \delta_{\dot{\delta}}^{\alpha}-\delta_{\beta \delta} \delta_{r}^{\alpha}\right)
\end{aligned}
$$




$$
\begin{aligned}
& -\frac{1}{n-3}\left[M_{\beta \varepsilon} M_{\gamma}^{\varepsilon} \delta_{\delta}^{\alpha}-M_{\beta \varepsilon} M_{\delta}^{\varepsilon} \delta_{\gamma}^{\alpha}+g_{\beta \gamma} M_{\varepsilon}^{\alpha} M_{\delta}^{\S}-g_{\beta \delta} M_{\varepsilon}^{\alpha} M_{\gamma}^{\varepsilon}\right] \\
& +\frac{M_{\rho}^{\varepsilon} M_{\varepsilon}^{\rho}}{(n-2)(n-3)}\left(g_{\beta \gamma} \delta_{\delta}^{\alpha}-g_{\beta \delta} \delta_{\gamma}^{\alpha}\right),
\end{aligned}
$$

où

$$
B_{\beta \curlyvee}^{j k}=B_{\beta}^{j} B_{\curlyvee}^{k}, \quad B^{j k}=B_{\beta}^{j} B_{\curlyvee}^{k} g^{\beta \gamma} .
$$

Les équations (4.56) et (4.66) nous donnent finalement

$$
\begin{aligned}
& C_{\beta \curlyvee \delta}^{\alpha}=B_{i \uparrow \uparrow \delta}^{\alpha j k h} C_{j k h}^{i}-\frac{1}{n-3}\left[B_{i}^{h} B_{\beta \uparrow}^{j k} C_{j k h}^{j} \delta_{\delta}^{\alpha}-B_{i}^{h} B_{\beta \delta \delta}^{j k} C_{j k h}^{i} \delta_{\uparrow}^{\alpha}\right.
\end{aligned}
$$

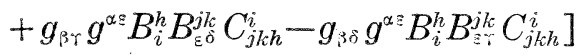

$$
\begin{aligned}
& +\frac{B_{i}^{h} B^{j k} C_{j k h}^{i}}{(n-2)(n-3)}\left(g_{\beta \gamma} \delta_{\delta}^{x}-g_{\beta \delta} \delta_{i}^{*}\right)
\end{aligned}
$$

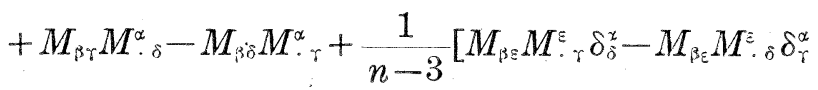

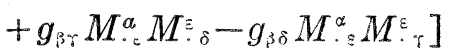

$$
\begin{aligned}
& -\frac{M_{\beta p}^{\varepsilon} M_{?}^{\rho}}{(n-2)(n-3)}\left(g_{\beta \gamma} \delta_{\delta}^{\alpha}-g_{\beta \delta} \delta_{\gamma}^{\alpha}\right),
\end{aligned}
$$

où

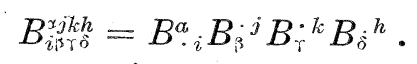

Ce sont les équations de Gauss pour une hypersurface dans un espace à connexion conforme normale. Ces équations ont été déjà obtenues pour le cas de l'espace de Riemann par un des présents auteurs. $\left({ }^{1}\right)$

Cela étant, passons à l'équation (4.57), ce qui peut être écrite aussi sous la forme

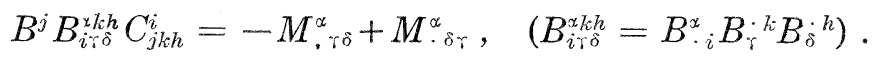

En contractant par rapport à $\alpha$ et $\gamma$, on trouve

$$
M_{. \alpha \delta}^{\alpha}=M_{. \delta \alpha}^{\alpha} .
$$

Donc les équations

$$
M_{\beta \gamma \delta}=M_{\beta \gamma \| \tilde{o}}-g_{\hat{r} \hat{\delta}}\left[\frac{1}{n-1} \frac{\partial g^{\varepsilon p} H_{\varepsilon p}}{\partial u^{\tau}}+I_{j k}^{0} B^{j} B_{r}^{\cdot k}\right]
$$

(1) K. Yano (3). 
nous donnent

$$
\begin{gathered}
M_{\cdot \alpha \delta}^{\alpha}=-\left[\frac{1}{n-1} \frac{\partial g^{\varepsilon \rho} H_{\varepsilon \rho}}{\partial u^{\delta}}+I I_{j k}^{0} B^{j} B_{\delta}^{\cdot k}\right], \\
M_{\cdot \delta \alpha}^{\alpha}=M_{\cdot \delta \mid \alpha}^{\alpha}-(n-1)\left[\frac{1}{n-1} \frac{\partial g^{\varepsilon \rho} H_{\varepsilon \rho}}{\partial u^{\delta}}+I_{j k}^{0} B^{j} B_{\delta} \cdot k\right],
\end{gathered}
$$

d'où

$$
\frac{1}{n-1} \frac{\partial g^{\varepsilon \rho} H_{\varepsilon \rho}}{\partial u^{\delta}}+I_{j k}^{0} B^{j} B_{\delta}^{\cdot k}=\frac{1}{n-2} M_{\delta \| \alpha}^{\alpha} .
$$

Donc l'équation (4.70) devient

$$
M_{\beta \gamma \delta}=M_{\beta \gamma \| \delta}-\frac{1}{n-2} g_{\beta \delta} M_{r_{\gamma / \alpha}^{\alpha}}^{\alpha}
$$

et les équations (4.68) nous donnent

(4.72) $\quad B^{j} B_{\cdot i}^{\alpha} B_{\gamma \delta}^{k h} C_{j k h}^{i}=-M_{\cdot \curlyvee \| \delta}^{\alpha}+M_{\cdot \delta \| \curlyvee}^{\alpha}+\frac{1}{n-2}\left[M_{\cdot \curlyvee \| \S}^{\varepsilon} \delta_{\delta}^{\alpha}-M_{\delta \| \varepsilon}^{\S} \delta_{\gamma}^{\alpha}\right]$.

Ce sont les équations de Codazzi pour l'hypersurface dans un espace à connexion normale. Dans le cas de Riemann, elles ont été aussi obtenues par un des présents auteurs. $\left({ }^{1}\right)$

Chapitre 5. Propriètés des courbes sur les hypersurfaces.

Considérons une courbe $C: u^{\lambda}(\tau)$ sur l'hypersurface, et définissons un point $S$ et une sphère $S$ respectivement par les équations suivantes: (i) (i)

$$
\begin{aligned}
& \underset{(\dot{0})}{S}=\frac{d \tau}{d \sigma} A_{\dot{0}}, \\
& \underset{\text { (i) }}{S}=\frac{\delta}{\delta \tau}, \underset{(\dot{0})}{S}=\frac{d}{d \tau} \underset{(\dot{0})}{S-(}-\left(\frac{d}{d \tau} \underset{(\dot{0})}{S} A_{\dot{n}}\right) A_{\dot{n}},
\end{aligned}
$$

où le paramètre $\sigma$ a été choisi de manière qu'on ait

$$
G_{\alpha_{3},} \frac{d u^{\alpha}}{d \sigma} \frac{d u^{\beta}}{d \sigma}=1
$$

(1) K. Yano (4). 
le long de la courbe. Si l'on a

$$
\left(\frac{\delta}{\delta \tau} \underset{(\mathrm{i})}{S}\right)^{2}=0
$$

le long de la courbe, on appelle $\tau$ le paramètre projectif( $\left.{ }^{1}\right)$ de la courbe considérée sur l'hypersurface.

Quand on regarde la courbe $C$ comme étant une courbe dans l'espace ambiant, le paramètre projectif $t$ est défini par les équations

$$
\begin{aligned}
& \underset{(0)}{S}=\frac{d t}{d s} A_{0}, \\
& \underset{(1)}{S}=\frac{d}{d t} \underset{(0)}{S}, \\
& \left(\frac{d}{d t} S_{(1)}^{S}\right)^{2}=0,
\end{aligned}
$$

où le paramètre conforme $s$ est défini par

$$
G_{j k} \frac{d u^{j}}{d s} \frac{d u^{l}}{d s}=1
$$

par conséquent, par

$$
\frac{d s}{d \sigma}=a^{\frac{1}{n-1}} .
$$

Grâce à la relation $A_{n} \frac{d}{d t} \underset{(0)}{S}=0$, on a successivement

$$
\begin{aligned}
& \underset{(\dot{0})}{S}=\frac{d \tau}{d t} \underset{(0)}{S}, \\
& \underset{\text { (i) }}{S}=\frac{\frac{d^{2} \tau}{d t^{2}}}{\frac{d \tau}{d t}} \underset{(0)}{S}+\underset{(1)}{S}, \\
& \frac{\delta}{\delta t} \underset{\text { (i) }}{S}=\left[\frac{\frac{d^{3} \tau}{d t^{3}}}{\left(\frac{d \tau}{d t}\right)^{2}}-\frac{\left(\frac{d^{2} \tau}{d t^{2}}\right)^{2}}{\left(\frac{d \tau}{d t}\right)^{3}}\right] \underset{(0)}{S}+\frac{\frac{d^{2} \tau}{d t^{2}}}{\left(\frac{d \tau}{d t}\right)^{2}} \underset{(1)}{S}
\end{aligned}
$$

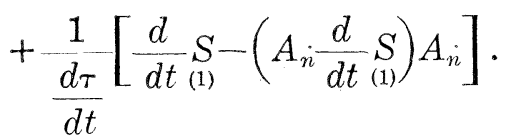

(1) K. Yano (2). 
En substituant (5.10) dans (5.4) et en tenant compte des relations $\underset{(0)}{S^{2}}=0, \underset{(1)}{S^{2}}=1,\left(\frac{d}{d t} \underset{\text { (1) }}{S}\right)^{2}=0, \underset{(0)(1)}{S S}=0, \underset{(0)}{S \frac{d}{d t}(1)} S=-1, \underset{(1)}{S} \frac{d}{d t} \underset{(1)}{S}=0 \quad$ et $\underset{(0)}{S} A_{\dot{n}}=\underset{(1)}{S} A_{\dot{n}}=0$, on obtient

$$
\left.\frac{\left(\frac{d^{2} \tau}{d t^{2}}\right)^{2}}{\left(\frac{d \tau}{d t}\right)^{4}}-\frac{1}{\left(\frac{d \tau}{d t}\right)^{2}}\left(\mathrm{~A}_{i} \frac{d}{d t} S\right)_{(1)}\right)^{2}-\frac{2}{\left(\frac{d \tau}{d t}\right)}\left[\frac{\frac{d^{3} \tau}{d t^{3}}}{\left(\frac{d \tau}{d t}\right)^{2}}-\frac{\left(\frac{d^{2} \tau}{d t^{2}}\right)^{2}}{\left(\frac{d \tau}{d t}\right)^{3}}\right]=0,
$$

d'où

$$
\frac{\frac{d^{3} \tau}{d t^{3}}}{\frac{d \tau}{d t}}-\frac{3}{2}\left(\frac{\frac{d^{2} \tau}{d t^{2}}}{\frac{d \tau}{d t}}\right)^{2}+\frac{1}{2}\left(A_{i} \frac{d}{d t} S\right)_{(1)}^{2}=0
$$

On voit donc que si la courbe $C$ sur l'hypersurface est en contact du second ordre avec la sphère $A_{\dot{n}}$ le long de la courbe, c'est-à-dire si l'on a $A_{\dot{n}} \frac{d}{d t} \underset{(1)}{S}=0$ le long de la courbe, les paramètres projectifs $\tau$ et $t$ coïncident à une transformation homographique près.

Mais, on a, d'autre part,

$$
\begin{aligned}
A_{\dot{n}} \frac{d}{d t} \underset{(1)}{S}=-\left(\frac{d A_{\dot{n}}}{d t}\right) \underset{(1)}{S}= & -\left(I I_{\dot{n} \alpha}^{0} \frac{d u^{\alpha}}{d t} A_{0}+I I_{\dot{n} \alpha}^{\beta} \frac{d u^{\alpha}}{d t} A_{\beta}\right) \\
& \times\left(\frac{d^{2} t}{d \sigma^{2}} \frac{d \sigma}{d t} A_{0}+\frac{d u^{\alpha}}{d \sigma} A_{\alpha}\right) \\
= & M_{\alpha \beta} \frac{d u^{\alpha}}{d t} \frac{d u^{\beta}}{d \sigma}
\end{aligned}
$$

en tenant compte des relations

$$
\underset{(0)}{S}=\frac{d t}{d \sigma} A_{0}, \quad \underset{(1)}{S}=\frac{d}{d t} \underset{(0)}{S}=\frac{d^{2} t}{d \sigma^{2}} \frac{d \sigma}{d t} A_{\dot{0}}+\frac{d u^{\alpha}}{d \sigma} A_{\alpha},
$$

en conséquence, (5.11) devient

$$
\{\tau, t\}+\frac{1}{2}\left(M_{\alpha \beta} \frac{d u^{\alpha}}{d t} \begin{array}{c}
d u^{\beta} \\
d \sigma
\end{array}\right)^{2}=0,
$$


où nous avons posé

$$
\{\tau, t\}=\frac{\frac{d^{3} \tau}{d t^{3}}}{\frac{d \tau}{d t}}-\frac{3}{2}\left(\frac{\frac{d^{2} \tau}{d t^{2}}}{\frac{d \tau}{d t}}\right)^{2}
$$

Les équations $M_{\alpha_{3}}=0$ représentant le fait que notre hypersurface est totalement ombiliquée (Voir le Chapitre 8), nous avons le théorème:

Si une courbe est tracée sur une hypersurface totalement ombiliquée, le paramètre projectif $t$ sur la courbe regardée comme celle dans l'espace ambiant et le paramètre projectif $\tau$ sur la courbe regardée comme celle sur l'hypersurface coüncident à une transformation homographique près.

Si l'on prend un paramètre projectif $t$, les sphères

$$
\underset{(0)}{S}=\frac{d t}{d s} A_{0}, \underset{(1)}{S}=\frac{d}{d t} \underset{(0)}{S}, \underset{(2)}{S}=\frac{d}{d t} \underset{(1)}{S}
$$

satisfont aux relations

$$
\left\{\begin{array}{ccc}
\underset{(0)(0)}{S S} S=0, & \underset{(0)(1)}{S S}=0, & \underset{(0)(2)}{S S}=-1 \\
& \underset{(1)(1)}{S S}=1, & \underset{(1)(2)}{S}=0, \\
& \underset{(2)(2)}{S} \underset{(S)}{S}=0 .
\end{array}\right.
$$

Cela étant, les équations de Frenet s’écrivent

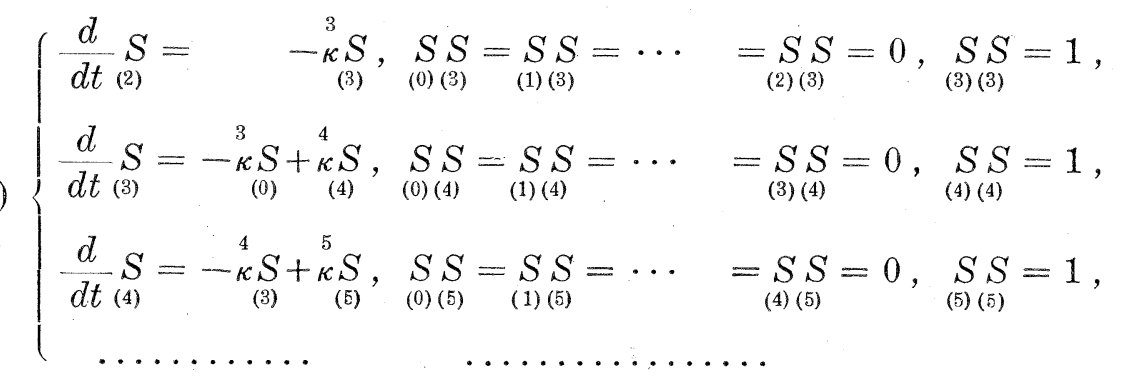

Quand on effectue une transformation homographique

$$
t^{\prime}=\frac{a t+b}{c t+d},
$$

du paramètre projectif $t$, les points et les sphères $\underset{(0)}{S}, \underset{(1)}{S}, \cdots$ se changent respectivement en $\underset{(0)}{S^{\prime}}, \underset{(1)}{S^{\prime}}, \cdots$. Nous allons, en passant, considérer les relations qui existent entre elles. 
On a tout d'abord

$$
\underset{(0)}{S^{\prime}}=\frac{d t^{\prime}}{d t} \underset{(0)}{S}
$$

donc, en dérivant successivement, on obtient

$$
\begin{aligned}
& \underset{(1)}{S^{\prime}}=\frac{d^{2} t^{\prime}}{d t^{2}} d t \underset{(0)}{S}+\underset{(1)}{S} \\
& \underset{(2)}{S^{\prime}}=\left[\frac{d^{3} t^{\prime}}{d t^{3}}\left(\frac{d t}{d t^{\prime}}\right)^{2}+\frac{d^{2} t^{\prime}}{d t^{2}} \frac{d^{2} t}{d t^{\prime 2}}\right] \underset{(0)}{S}+\frac{d^{2} t^{\prime}}{d t^{2}}\left(\frac{d t}{d t^{\prime}}\right)^{2} \underset{(1)}{S}+\frac{d t}{d t^{\prime}} \underset{(2)}{S}, \\
& \left.\frac{d}{d t^{\prime}} \underset{(2)}{S^{\prime}}=\left[\frac{d^{4} t^{\prime}}{d t^{4}}\left(\frac{d t}{d t^{\prime}}\right)^{3}+3 \frac{d^{3} t^{\prime}}{d t^{3}} \frac{d^{2} t}{d t^{\prime 2}} \frac{d t}{d t^{\prime}}+\frac{d^{2} t^{\prime}}{d t^{2}} d t^{3} t\right]\right]_{(0)}^{S} \\
& +\left[2 \frac{d^{3} t^{\prime}}{d t^{3}}\left(\frac{d t}{d t^{\prime}}\right)^{3}+3 \frac{d^{2} t^{\prime}}{d t^{2}} \frac{d^{2} t}{d t^{\prime 2}} \frac{d t}{d t^{\prime}}\right] \underset{(1)}{S} \\
& +\left[\frac{d^{2} t^{\prime}}{d t^{2}}\left(\frac{d t}{d t^{\prime}}\right)^{3}+\frac{d^{2} t}{d t^{\prime 2}}\right] \underset{(2)}{S}+\left(\frac{d t}{d t^{\prime}}\right)^{2} \frac{d}{d t} \underset{(2)}{S} .
\end{aligned}
$$

Or, les relations

$$
\left\{\begin{array}{l}
\frac{d^{4} t^{\prime}}{d t^{4}}\left(\frac{d t}{d t^{\prime}}\right)^{3}+3 \frac{d^{3} t^{\prime}}{d t^{3}} \frac{d^{2} t}{d t^{\prime 2}} \frac{d t}{d t^{\prime}}+\frac{d^{2} t^{\prime}}{d t^{2}} \frac{d^{3} t}{d t^{\prime 3}}=0 \\
2 \frac{d^{3} t^{\prime}}{d t^{\prime 3}}\left(\frac{d t}{d t^{\prime}}\right)^{3}+3 \frac{d^{2} t^{\prime} d^{2} t}{d t^{2}} \frac{d t}{d t^{\prime 2}} \frac{d t}{d t^{\prime}}=0 \\
\frac{d^{2} t^{\prime}}{d t^{2}}\left(\frac{d t}{d t^{\prime}}\right)^{3}+\frac{d^{2} t}{d t^{\prime 2}}=0
\end{array}\right.
$$

qui résultent du (5.17), nous donnent

$$
\frac{d}{d t^{\prime}} \underset{(2)}{S^{\prime}}=\left(\frac{d t}{d t^{\prime}}\right)^{2} \frac{d}{d t} \underset{(2)}{S},
$$

d'où

$$
\stackrel{3}{\kappa^{\prime}}=\left(\frac{d t}{d t^{\prime}}\right)^{23} \kappa
$$

en raison des relations

$$
\left(\frac{d}{d t^{\prime}} S\right)_{(2)}^{S}=\kappa^{\prime 2} \quad \text { et } \quad\left(\frac{d}{d t} \underset{(2)}{S}\right)^{2}=\kappa^{2} .
$$


Donc on voit que

$$
\underset{(3)}{S^{\prime}}=\underset{(3)}{S}
$$

Cela étant, les équations de Frenet

$$
\begin{gathered}
\frac{d}{d t} \underset{(3)}{S}=-\kappa_{(0)}^{3} \underset{(4)}{S}+\underset{(4)}{S}, \\
\frac{d}{d t^{\prime}} \underset{(3)}{S^{\prime}}=-\kappa^{\prime} \underset{(0)}{S^{\prime}+\kappa^{\prime} \underset{(4)}{S^{\prime}},}
\end{gathered}
$$

montrent que

$$
\stackrel{4}{\kappa}^{\prime}=\frac{d t}{d t^{\prime}} \stackrel{4}{\kappa} \quad \text { et } \quad \underset{(4)}{S^{\prime}}=\underset{(4)}{S}
$$

En utilisant la relation (5.23), on peut définir une quantité invariante par rapport à la transformation homographique (5.17) de paramètre projectif.

On obtient de (5.23)

$$
\frac{d_{\kappa^{\prime}}^{3}}{d t^{\prime}}=2 \frac{d^{2} t}{d t^{\prime 2}} \frac{d t^{3}}{d t^{\prime}} \kappa^{3}+\frac{d_{\kappa}^{3}}{d t}\left(\frac{d t}{d t^{\prime}}\right)^{3}
$$

$$
\frac{d^{2} \kappa^{\prime}}{d t^{\prime 2}}=2\left[\frac{d^{3} t}{d t^{\prime 3}} \frac{d t}{d t^{\prime}}+\left(\frac{d^{2} t}{d t^{\prime 2}}\right)^{2}\right]^{3} \kappa+5 \frac{d^{2} t}{d t^{\prime 2}}\left(\frac{d t}{d t^{\prime}}\right)^{2} \frac{d^{3}}{d t}+\frac{d^{2} \kappa}{d t^{2}}\left(\frac{d t}{d t^{\prime}}\right)^{4}
$$

d'où

$$
\frac{d^{2} \kappa^{\prime}}{d t^{\prime 2}}=5\left(\frac{d^{2} t}{d t^{\prime 2}}\right)^{2}{ }^{3}+5 \frac{d^{2} t}{d t^{\prime 2}}\left(\frac{d t}{d t^{\prime}}\right)^{2} \frac{d_{\kappa}^{3}}{d t}+\frac{d^{2}{ }^{3}}{d t^{2}}\left(\frac{d t}{d t^{\prime}}\right)^{4}
$$

grâce à l'équation

$$
\frac{d^{3} t}{d t^{\prime 3}} \frac{d t}{d t^{\prime}}=\frac{3}{2}\left(\frac{d^{2} t}{d t^{\prime 2}}\right)^{2}
$$

Donc, nous avons

$$
\frac{1}{\kappa_{\kappa^{\prime}, 2}^{3}} \frac{d^{2} \kappa^{\prime}}{d t^{\prime 2}}-\frac{5}{4} \frac{1}{{\stackrel{3}{\left(\kappa^{\prime}\right)^{3}}}^{3}}\left(\frac{d^{3} \kappa^{\prime}}{d t^{\prime}}\right)^{2}=\frac{1}{\underset{(\kappa)^{2}}{3}} \frac{d^{2} \kappa}{d t^{2}}-\frac{5}{4} \frac{1}{\underset{(\kappa)^{3}}{3}}\left(\frac{d \kappa}{d t}\right)^{2},
$$

par conséquent, on voit que la quantité

$$
\frac{1}{(\kappa)^{2}} \frac{d^{2} \kappa}{d t^{2}}-\frac{5}{4} \frac{1}{(\kappa)^{3}}\left(\frac{d_{\kappa}^{3}}{d t}\right)^{2}
$$


est invariante par rapport à la transformation homographique de paramètre projectif $t$.

Cela dit, nous allons, dans la suite, chercher la relation entre les points et les sphères $S S \cdots$ sur l'hypersurface et $S S \cdots$ dans l'éspace ambiant. On a
(i), (i),
(0), (1),

$(5.30) \underset{(0)}{S}=\frac{d \tau}{d t} \underset{(0)}{S}$

(5.31) $\underset{\text { (i) }}{S}=\frac{d^{2} \tau}{d t^{2}} \frac{d t}{d \tau} \underset{(0)}{S}+\underset{(1)}{S}$

(5.32) $\left.\underset{(\text { (2) }}{S}=\left[\frac{d^{3} \tau}{d t^{3}}\left(\frac{d t}{d \tau}\right)^{2}+\frac{d^{2} \tau}{d t^{2}} \frac{d^{2} t}{d \tau^{2}}\right]_{(0)}^{S}+\frac{d^{2} \tau}{d t^{2}}\left(\frac{d t}{d \tau}\right)_{(1)}^{2} \underset{(2)}{S}+\underset{(2)}{S-\left(A_{\dot{n}} S\right)} A_{\dot{n}}\right] \frac{d t}{d \tau}$,

d'où l'on tire encore

$$
\begin{aligned}
& \frac{\delta}{\delta \tau} \underset{(\dot{2})}{S}=\frac{d}{d \tau}\left[\frac{d^{3} \tau}{d t^{3}}\left(\frac{d t}{d \tau}\right)^{2}+\frac{d^{2} \tau}{d t^{2}} \frac{d t^{2}}{d \tau^{2}}\right] \underset{(0)}{S} \\
& +\left[\frac{d^{3} \tau}{d t^{3}}\left(\frac{d t}{d \tau}\right)^{3}+3 \frac{d^{2} \tau}{d t^{2}} \frac{d^{2} t}{d \tau^{2}} \frac{d t}{d \tau}+\frac{d^{3} \tau}{d t^{3}}\left(\frac{d t}{d \tau}\right)^{3}\right]_{(1)}^{S} \\
& +\left[\underset{(2)}{S-}\left(A_{i} \underset{(2)}{S}\right) A_{\dot{n}}\right]\left[\frac{d^{2} t}{d \tau^{2}}+\frac{d^{2} \tau}{d t^{2}}\left(\frac{d t}{d \tau}\right)^{3}\right] \\
& +\left[\frac{d}{d t} \underset{(2)}{S}-\left(A_{\dot{n}} \frac{d}{d t} \underset{(\mathrm{(})}{S}\right) A_{\dot{n}}-\left(A_{\dot{n}} \underset{(2)}{S)} \frac{d A_{\dot{n}}}{d t}\right]\left(\frac{d t}{d \tau}\right)^{2} .\right.
\end{aligned}
$$

Or comme nous avons les identités

$$
\begin{gathered}
\frac{d^{2} \tau}{d t^{2}}+\frac{d t^{2}}{d \tau^{2}}\left(\frac{d \tau}{d t}\right)^{3}=0 \\
\{\tau, t\}+\{t, \tau\}\left(\frac{d \tau}{d t}\right)^{2}=0,
\end{gathered}
$$

on tire de $(5,33)$

$$
\begin{aligned}
& -\dot{\beta}_{(\dot{3})}^{\dot{3}} S=-\frac{1}{2}\left(\frac{d t}{d \tau}\right)^{2} \frac{d}{d t}\left[M_{\alpha \beta} \frac{d u^{\alpha}}{d \sigma} \frac{d u^{\beta}}{d \sigma} \frac{d \sigma}{d t}\right]_{(0)}^{2} \\
& -\left(M_{\alpha \beta} \frac{d u^{\alpha}}{d \sigma} \frac{d u^{\beta}}{d \sigma}\right)^{2}\left(\frac{d \sigma}{d \tau}\right)^{2} \underset{(1)}{S} \\
& +\left[-\underset{(3)}{\kappa} S-\left(A_{\dot{n}} \underset{(2)}{S}\right) \frac{d A_{\dot{n}}}{d t}+{ }^{3}\left(A_{\dot{n}} \underset{(3)}{S} A_{\dot{n}}\right]\left(\frac{d t}{d \tau}\right)^{2},\right.
\end{aligned}
$$


ce qui peut être encore écrite sous la forme:

$$
\begin{aligned}
& -\underset{(\dot{3})}{\dot{3}} \underset{(\dot{3})}{S}=-\frac{1}{2}\left(\frac{d t}{d \tau}\right)^{2} \frac{d}{d t}\left(A_{\dot{n}} \underset{(2)}{S}\right)_{(0)}^{2} \underset{(0)}{S}-\left(A_{i} S\right)^{2}\left(\frac{d t}{d \tau}\right)^{2} \underset{(1)}{S}
\end{aligned}
$$

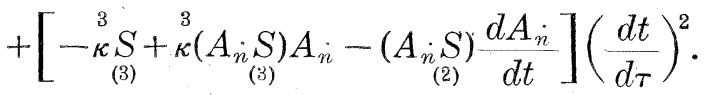

Ces équations nous donnent

$$
\begin{aligned}
& (\stackrel{\dot{3}}{\kappa})^{2}=\left[\stackrel{3}{(\kappa)^{2}}-(\stackrel{3}{\kappa})^{2}\left(A_{\dot{n}} \underset{(3)}{S}\right)^{2}+2 \stackrel{3}{\kappa}\left(A_{\dot{n}} \underset{(2)}{S}\right)\left(\frac{d A_{\dot{n}}}{d t} \underset{(3)}{S}\right)\right. \\
& \left.+\left(A_{\dot{n}} S_{(2)}\right)^{2} \frac{d A_{\dot{n}}}{d t} \frac{d A_{\dot{n}}}{d t}-\left(A_{\dot{n}} S\right)_{(2)}^{4}\right]\left(\frac{d t}{d \tau}\right)^{4} .
\end{aligned}
$$

Donc en posant

$$
\begin{aligned}
& K^{2}=(\stackrel{3}{\kappa})^{2}-(\stackrel{3}{\kappa})^{2}\left(A_{\dot{n}} \underset{(3)}{S}\right)^{2}+\stackrel{3}{2}_{\kappa}\left(A_{\dot{n}} \underset{(2)}{S}\right)\left(\frac{d A_{\dot{n}}}{d t} \underset{(3)}{S}\right) \\
& +\left(A_{\dot{n}} S_{(2)}\right)^{2} \frac{d A_{\dot{n}}}{d t} \frac{d A_{\dot{n}}}{d t}-\left(A_{\dot{n}} S_{(2)}\right)^{4}
\end{aligned}
$$

on obtient

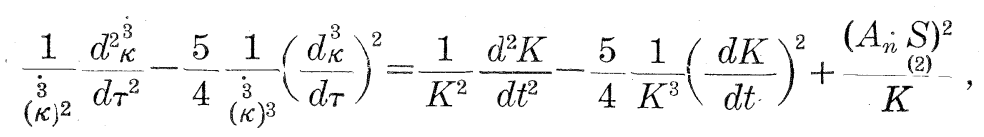

et

$$
\begin{aligned}
\underset{(\dot{3})}{S}= & \left\{\frac{\kappa}{K} \underset{(3)}{S}+\frac{\underset{(2)}{\left(A_{\dot{n}} S\right)}}{K} \frac{d A_{\dot{n}}}{d t}-\frac{\kappa}{K}\left(A_{\dot{n}} S_{(3)}\right) A_{\dot{n}}\right\} \\
& +\frac{1}{K}\left(M_{\alpha_{\beta}} \frac{d u^{\alpha}}{d \sigma} \frac{d u^{\beta}}{d \sigma}\right)^{2}\left(\frac{d \sigma}{d t}\right)_{(1)}^{2}+\frac{1}{2} \frac{1}{K} \frac{d}{d t}\left(M_{\alpha \beta} \frac{d u^{\alpha}}{d \sigma} \frac{d u^{\beta}}{d \sigma} \frac{d \sigma}{d t}\right)_{(0)}^{2} .
\end{aligned}
$$

Chpitre 6. Circonférences généralisées sur les hypersurfaces.

La circonférence généralisée ${ }^{1}$ ) sur l'hypersurface peut être définie comme une courbe sur l'hypersurface pour laquelle nous avons $\stackrel{\dot{3}}{\kappa}=0$. Donc, pour une telle courbe nous avons de (5.35)

(1) K. Yano. (1). 
(6.1)

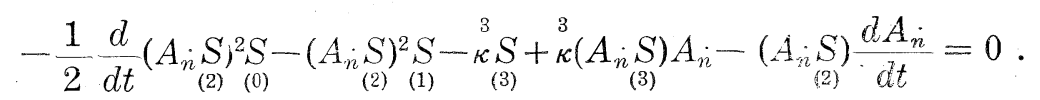

Cela étant, on a, d'autre part,

$$
\begin{aligned}
& \underset{(0)}{S}=\frac{d t}{d s} A_{0}, \\
& \underset{(1)}{S}=\frac{\frac{d^{2} t}{d s^{2}}}{\frac{d t}{d s}} A_{0}+\frac{d A_{0}}{d s} \\
& \underset{(2)}{S}=\left[\frac{\frac{d^{3} t}{d s^{3}}}{\left(\frac{d t}{d s}\right)^{2}}-\frac{\left(\frac{d^{2} t}{d s^{2}}\right)^{2}}{\left(\frac{d t}{d s}\right)^{3}}\right] A_{0}+\frac{\frac{d^{2} t}{d s^{2}}}{\left(\frac{d t}{d s}\right)^{2}} \frac{d A_{0}}{d s}+\frac{1}{\frac{d t}{d s}} \frac{d^{2} A_{0}}{d s^{2}} \\
& -{ }_{(3)}^{3} S=\left[\frac{\frac{d^{4} t}{d s^{4}}}{\left(\frac{d t}{d s}\right)^{3}}-\frac{4 \frac{d^{3} t}{d s^{3}} \frac{d^{2} t}{d s^{2}}}{\left(\frac{d t}{d s}\right)^{4}}+3 \frac{\left(\frac{d^{2} t}{d s^{2}}\right)^{3}}{\left(\frac{d t}{d s}\right)^{5}}\right] A_{0} \\
& +\left[2 \frac{\frac{d^{3} t}{d s^{3}}}{\left(\frac{d t}{d s}\right)^{3}}-\frac{3\left(\frac{d^{2} t}{d s^{2}}\right)^{2}}{\left(\frac{d t}{d s}\right)^{4}}\right] \frac{d A_{0}}{d s}+\frac{1}{\left(\frac{d t}{d s}\right)^{2}} \frac{d^{3} A_{0}}{d s^{3}}
\end{aligned}
$$

d'où, en utilisant la notation

$$
\{t, s\}=\frac{\frac{d^{3} t}{d s^{3}}}{\frac{d t}{d s}}-\frac{3}{2}\left(\frac{\frac{d^{2} t}{d s^{2}}}{\frac{d t}{d s}}\right)^{2}
$$

on obtient

$$
-{ }_{-\infty}^{3} \underset{(3)}{S}=\frac{\frac{d}{d s}\{t, s\}}{\left(\frac{d t}{d s}\right)^{2}} A_{0}+\frac{2\{t, s\}}{\left(\frac{d t}{d s}\right)^{2}} \frac{d A_{0}}{d s}+\frac{1}{\left(\frac{d t}{d s}\right)^{2}} \frac{d^{3} A_{0}}{d s^{3}}
$$


Par conséquent, en substituant les relations

$$
\begin{aligned}
& \frac{d A_{0}}{d s}= \frac{d u^{i}}{d s} A_{i}, \\
& \frac{d^{2} A_{0}}{d s^{2}}= I_{j k}^{0} \frac{d u^{j}}{d s} \frac{d u^{k}}{d s} A_{0}+\left(\frac{d^{2} u^{i}}{d s^{2}}+I I_{j k}^{i} \frac{d u^{j}}{d s} \frac{d u^{k}}{d s}\right) A_{i}+A_{\infty}, \\
& \frac{d^{3} A_{0}}{d s^{3}}= {\left[\frac{d}{d s}\left(I I_{j k}^{0} \frac{d u^{j}}{d s} \frac{d u^{k}}{d s}\right)+I_{j k}^{0} a^{j} \frac{d u^{k}}{d s}\right] A_{0} } \\
&+\left(\frac{d a^{i}}{d s}+I I_{j k}^{i} a^{j} \frac{d u^{k}}{d s}+I_{j k}^{0} \frac{d u^{j}}{d s} \frac{d u^{k}}{d s} \frac{d u^{i}}{d s}+I I_{\infty k}^{i} \frac{d u^{k}}{d s}\right) A_{i}, \\
&\{t, s\}=\frac{1}{2} G_{j k} a^{j} a^{k}-I_{j k}^{0} \frac{d u^{j}}{d s} \frac{d u^{k}}{d s},
\end{aligned}
$$

où

$$
a^{i}=\frac{d^{2} u^{i}}{d s^{2}}+\Pi_{j k}^{i} \frac{d u^{j}}{d s} \frac{d u^{k}}{d s}
$$

dans (6.2), on trouve

$$
\begin{gathered}
-\underset{(3)}{\kappa_{(3)}}=\frac{1}{\left(\frac{d t}{d s}\right)^{2}}\left[\frac{1}{2}\left(G_{j k} a^{j} a^{k}\right)+I_{j k}^{0} a^{j} \frac{d u^{k}}{d s}\right] A_{0} \\
+\frac{1}{\left(\frac{d t}{d s}\right)^{2}}\left[\frac{d a^{i}}{d s}+I_{j k}^{i} a^{j} \frac{d u^{k}}{d s}+\left(G_{j k} a^{j} a^{k}-I_{j k}^{0} \frac{d u^{j}}{d s} \frac{d u^{k}}{d s}\right) \frac{d u^{i}}{d s}\right. \\
\left.+I_{\infty<k}^{i} \frac{d u^{k}}{d s}\right] A_{i} .
\end{gathered}
$$

Les équations (6.1) et (6.3) nous donnent

$$
\begin{aligned}
& {\left[\frac{1}{2} \frac{d}{d s}\left(G_{j k} a^{j} a^{k}\right)+I I_{j k}^{0} a^{j} \frac{d u^{k}}{d s}\right] A_{0}} \\
& +\left[\frac{d a^{i}}{d s}+I I_{j k}^{i} a^{j} \frac{d u^{k}}{d s}+\left(G_{j k} a^{j} a^{k}-I I_{j k}^{0} \frac{d u^{j}}{d s} \frac{d u^{k}}{d s}\right) \frac{d u^{i}}{d s}\right. \\
& \left.+I I_{\infty k}^{i} \frac{d u^{k}}{d s}\right]\left[A_{i}-\left(A_{i} A_{\dot{n}}\right) A_{\dot{n}}\right]
\end{aligned}
$$




$$
\begin{aligned}
& -M_{\alpha_{\beta}} \frac{d u^{\alpha}}{d \sigma} \frac{d u^{\beta}}{d \sigma} \frac{d \sigma}{d s} \frac{d A_{\dot{n}}}{d s}-\left(M_{\alpha_{\beta}} \frac{d u^{\alpha}}{d \sigma} \frac{d u^{\beta}}{d \sigma}\right)^{2}\left(\frac{d \sigma}{d s}\right)^{2}\left(\frac{\frac{d^{2} t}{d s^{2}}}{\frac{d t}{d s}} A_{0}+\frac{d u^{i}}{d s} A_{i}\right) \\
& -\left(M_{\alpha_{\beta}} \frac{d u^{\alpha}}{d \sigma} \frac{d u^{\beta}}{d \sigma}\right) \frac{d \sigma}{d s} \frac{d}{d s}\left(M_{\alpha_{\beta}^{\beta}} \frac{d u^{\alpha}}{d \sigma} \frac{d u^{\beta}}{d \sigma} \frac{d \sigma}{d t}\right) \frac{d t}{d s} A_{0}=0 .
\end{aligned}
$$

$D^{\prime}$ autre part, $\frac{d A_{\dot{n}}}{d s}$ étant donné par

$$
\begin{aligned}
\frac{d A_{\dot{n}}}{d s} & =\left(\frac{d N^{0}}{d s}+I I_{j k}^{0} N^{j} \frac{d u^{k}}{d s}\right) A_{0}+\left(\frac{d N^{i}}{d s}+I_{j k}^{i} N^{j} \frac{d u^{k}}{d s}+N^{0} \frac{d u^{i}}{d s}\right) A_{i} \\
& =\left(\frac{d N^{0}}{d s}+I_{j k}^{0} N^{j} \frac{d u^{k}}{d s}\right) A_{0}-B_{\alpha}^{\cdot i} M_{-\beta}^{\alpha} \frac{d u^{\beta}}{d s} A_{i},
\end{aligned}
$$

où

$$
N^{0}=\frac{a^{-\frac{2}{n-1}}}{n-1} G^{\alpha_{3}} N_{\alpha_{\beta}},
$$

(6.4) devient

(6.6) $\frac{1}{2} \frac{d}{d s}\left(G_{j k} a^{j} a^{k}\right)+I I_{j k}^{0} a^{j} \frac{d u^{k}}{d s}-M_{\alpha_{\beta}} \frac{d u^{\alpha}}{d \sigma} \frac{d u^{\beta}}{d \sigma} \frac{d \sigma}{d s}\left(\frac{d N^{0}}{d s}+I I_{j k}^{0} N^{j} \frac{d u^{k}}{d s}\right)$

$$
\begin{array}{r}
-\left(M_{\alpha \beta} \frac{d u^{\alpha}}{d \sigma} \frac{d u^{\beta}}{d \sigma}\right)\left(\frac{d \sigma}{d s}\right)^{3} \frac{d}{d \sigma}\left(M_{\gamma \delta} \frac{d u^{\gamma}}{d \sigma} \frac{d u^{\delta}}{d \sigma}\right) \\
-\left(M_{\alpha \beta} \frac{d u^{\alpha}}{d \sigma} \frac{d u^{\beta}}{d \sigma}\right)^{2} \frac{d^{2} \sigma}{d s^{2}} \frac{d \sigma}{d s} \\
-N^{0} N_{i}\left(\frac{d a^{i}}{d s}+I_{j k}^{i} a^{j} \frac{d u^{k}}{d s}+I_{\infty k}^{i} \frac{d u^{k}}{d s}\right)=0,
\end{array}
$$

(6.7) $\frac{d a^{i}}{d s}+\Pi_{j k}^{i} a^{j} \frac{d u^{k}}{d s}+\left(G_{j k} a^{j} a^{k}-I I_{j k}^{0} \frac{d u^{j}}{d s} \frac{d u^{k}}{d s}\right) \frac{d u^{i}}{d s}+I I_{\infty k}^{i} \frac{d u^{k}}{d s}$

$$
\begin{aligned}
& -N^{i} N_{j}\left(\frac{d a^{j}}{d s}+I_{k h}^{j} a^{k} \frac{d u^{h}}{d s}+I_{\propto h}^{j} \frac{d u^{h}}{d s}\right) \\
& +M_{\alpha \beta} \frac{d u^{\alpha}}{d \sigma} \frac{d u^{\beta}}{d \sigma}\left(\frac{d \sigma}{d s}\right)^{2} B_{\gamma}^{\cdot / i} M_{\delta}^{\curlyvee} \frac{d u^{\delta}}{d \sigma} \\
& -\left(M_{\alpha \beta} \frac{d u^{\alpha}}{d \sigma} \frac{d u^{\beta}}{d \sigma}\right)^{2}\left(\frac{d \sigma}{d s}\right)^{2} \frac{d u^{i}}{d s}=0 .
\end{aligned}
$$


Comme on peut, par un calcul direct, vérifier que l'équation (6.6) est une conséquence des équations (6.7), les équations (6.7) sont les équations différentielles de circonférences généralisées sur l'hypersurface.

Si l'on peut mener, dans une direction arbitrairement donnée, une circonférence généralisée de l'espace ambiant sur l'hypersurface, on a de (6.7)

$$
\begin{gathered}
N_{j}\left(\frac{d a^{j}}{d s}+I I_{k h}^{j} a^{k} \frac{d u^{h}}{d s}+I T_{\infty h}^{j} \frac{d u^{h}}{d s}\right)=0, \\
M_{\alpha \beta} \frac{d u^{\alpha}}{d \sigma} \frac{d u^{\beta}}{d \sigma}\left(\frac{d \sigma}{d s}\right)^{2} B_{\gamma}^{i} M_{\cdot \delta}^{\gamma} \frac{d u^{\delta}}{d s}-\left(M_{\alpha \beta} \frac{d u^{\alpha}}{d \sigma} \frac{d u^{\beta}}{d \sigma}\right)^{2}\left(\frac{d \sigma}{d s}\right)^{2} \frac{d u^{i}}{d s}=0,
\end{gathered}
$$

d'où

$$
M_{\alpha \beta}=0 \text {. }
$$

Donc, notre hypersurface doit être totalement ombiliquée.

Les équations (6.7) peuvent être écrites aussi sous la forme

$$
\begin{gathered}
B_{\cdot i}^{\alpha}\left[\frac{d a^{i}}{d s}+I_{j k}^{i} a^{j} \frac{d u^{k}}{d s}+\left(G_{j k} a^{j} a^{k}-I I_{j k}^{0} \frac{d u^{j}}{d s} \frac{d u^{k}}{d s}\right) \frac{d u^{i}}{d s}+I_{\infty k}^{i} \frac{d u^{k}}{d s}\right]+ \\
+a^{-\frac{2}{n-1}} M_{\curlyvee \delta} \frac{d u^{\gamma}}{d \sigma} \frac{d u^{\delta}}{d \sigma}\left(M_{\cdot \beta}^{\alpha} \frac{d u^{\beta}}{d \sigma}-M_{\curlyvee \delta} \frac{d u^{\curlyvee}}{d \sigma} \frac{d u^{\delta}}{d \sigma} \cdot \frac{d u^{\alpha}}{d \sigma}\right)=0
\end{gathered}
$$

Quand on regarde cette courbe comme étant une courbe sur l'hypersurface à connexion conforme induite, on a les équations différentielles

$$
\frac{d a^{\alpha}}{d \sigma}+I_{\beta \gamma}^{\alpha} a^{\beta} \frac{d u^{\gamma}}{d \sigma}+\left(G_{\beta r} a_{r}^{\beta} a^{\Upsilon}-I_{\beta \gamma}^{j} \frac{d u^{\beta}}{d \sigma} \frac{d u^{\Upsilon}}{d \sigma}\right) \frac{d u^{\alpha}}{d \sigma}+I_{\dot{\alpha} \gamma}^{\alpha} \frac{d u^{\Upsilon}}{d s}=0
$$

où

$$
a^{\alpha}=\frac{d^{2} u^{\alpha}}{d \sigma^{2}}+I_{\beta, r}^{\alpha} \frac{d u^{\beta}}{d \sigma} \frac{d u^{r}}{d \sigma}
$$

ceci peut être facilement vérifié en employant les relations $\left({ }^{1}\right)$

$$
\begin{gathered}
\frac{d u^{\alpha}}{d \sigma}=B_{\cdot i}^{\alpha} \frac{d u^{i}}{d s}, \\
a^{\alpha}=a^{\frac{1}{n-1}} B_{\cdot i}^{\alpha} a^{i}-\frac{\partial \log a^{-\frac{1}{n-1}}}{d u^{\beta}} G^{\alpha \beta}+\frac{d \log a^{-\frac{1}{n-1}}}{d \sigma} \frac{d u^{\alpha}}{d \sigma},
\end{gathered}
$$

(1) Y. Mutồ. (2). 
(6.12) $\frac{d a^{\alpha}}{d \sigma}+\Pi_{\beta \gamma}^{\alpha} a^{\beta} \frac{d u^{\gamma}}{d \sigma}+\left(G_{\beta \gamma} \alpha^{\beta} \alpha^{\gamma}-I I_{\beta \gamma}^{\dot{0}} \frac{d u^{\beta}}{d \sigma} \frac{d u^{r}}{d \sigma}\right) \frac{d u^{\alpha}}{d \sigma}+I_{\dot{\infty}^{\gamma}}^{\alpha} \frac{d u^{\gamma}}{d \sigma}$

$$
\begin{aligned}
=a^{\frac{2}{n-1}} B_{\cdot i}^{\alpha}\left[\frac{d a^{i}}{a s}+I_{j k}^{i} a^{j}-\frac{d u^{k}}{d s}\right. & \\
& \left.+\left(G_{j k} a^{j} a^{k}-I_{j k}^{0} \frac{d u^{j}}{d s} \frac{d u^{k}}{d s}\right) \frac{d u^{i}}{d s}+I_{\infty h}^{i} \frac{d u^{h}}{d s}\right] \\
& +\left(M_{{ }_{\beta}}^{\alpha} \frac{d u^{\beta}}{d \sigma}-M_{\gamma \delta} \frac{d u^{\tau}}{d \sigma} \frac{d u^{\delta}}{d \sigma} \frac{d u^{\alpha}}{d \sigma}\right) M_{\varepsilon x} \frac{d u^{\varepsilon}}{d \sigma} \frac{d u^{\alpha}}{d \sigma}
\end{aligned}
$$

Les équations (6.11) et (6.12) nous donnent encore

$$
\begin{aligned}
& a^{i}=a^{-\frac{1}{n_{\alpha}-1}} B_{\alpha^{i}} a^{\alpha}+a^{-\frac{1}{n-1}} G^{\alpha_{\beta}} B_{\alpha}{ }^{i} \frac{\partial \log a^{-\frac{1}{n-1}}}{\partial u^{\beta}} \\
& +a^{-\frac{2}{n-1}} N_{\alpha \beta} \frac{d u^{\alpha}}{d \sigma} \frac{d u^{\beta}}{d \sigma} N^{i}-a^{-\frac{1}{n-1}} \frac{d \log a^{-\frac{1}{n-1}}}{d \sigma} \frac{d u^{i}}{d s}, \\
& \frac{d}{d s} a^{i}+\Pi_{j k}^{i} a^{j} \frac{d u^{k}}{d s}+\left(G_{j k} a^{j} a^{k}-I_{j k}^{0} \frac{d u^{j}}{d s} \frac{d u^{k}}{d s}\right) \frac{d u^{i}}{d s}+\Pi_{\circ c k}^{i} \frac{d u^{k}}{d s} \\
& =a^{-\frac{2}{n-1}} B_{\alpha}^{i}\left[\frac{d}{d \sigma} a^{\alpha}+\Pi_{\beta \gamma}^{\alpha} a^{\beta} \frac{d u^{r}}{d \sigma}\right. \\
& +\left(G_{\beta \gamma} a^{\beta} a^{\gamma}-I_{\beta r}^{j} \frac{d u^{\beta}}{d \sigma} \frac{d u^{r}}{d \sigma}\right) \frac{d u^{\alpha}}{d \sigma}+I_{\infty \gamma}^{\alpha} \frac{d u^{r}}{d \sigma} \\
& \left.-\left(M_{\beta}^{\alpha} \frac{d u^{\beta}}{d \sigma}-M_{\gamma \delta \delta} \frac{d u^{\gamma}}{d \sigma} \frac{d u^{\delta}}{d \sigma} \frac{d u^{\alpha}}{d \sigma}\right) M_{\varepsilon x} \frac{d u^{\varepsilon}}{d \sigma} \frac{d u^{\gamma}}{d \sigma}\right] \\
& +\left[a^{-\frac{3}{n-1}} \frac{d \log a^{-\frac{1}{n-1}}}{d \sigma} N_{\beta r} \frac{d u}{d \sigma} \frac{d u}{d \sigma}\right. \\
& +a^{-\frac{3}{n-1}}\left(N_{\beta r, \delta}-I_{\beta \delta}^{\varepsilon} N_{\varepsilon \gamma}-\Pi_{\gamma \delta}^{\varepsilon} N_{\beta \varepsilon}\right) \frac{d u^{\beta}}{d \sigma} \frac{d u^{r}}{d \sigma} \frac{d u^{\delta}}{d \sigma} \\
& +3 a^{-\frac{3}{n-1}} N_{\beta \gamma} \frac{d u^{\beta}}{d \sigma} a^{\curlyvee}+a^{-\frac{3}{n-1} \partial \log a^{-\frac{1}{n-1}}} G^{\beta r} N_{\curlyvee \delta} \frac{d u^{\delta}}{d \sigma} \\
& \left.+\Pi_{j k}^{0} N^{j} \frac{d u^{k}}{d s}\right] N^{i}
\end{aligned}
$$




\section{Chapitire \%. Les courbes autoconcourantes.}

En considérant une congruences $\Gamma$ des sphères $A$ tangentes à une surface donnée dans un espace conforme, M. Haimovici( $\left.{ }^{1}\right)$ a défini la direction concourante le long de la courbe sur l'hypersurface et la courbe autoconcourante c'est-à-dire une courbe dont la tangente est concourante le long de la courbe elle-même.

La circonférence généralisée sur l'hypersurface que nous avons traitée dans le Chapitre précédent est une sorte de courbe autoconcourante, seulement, nous avons pris les sphères $A_{\dot{n}}$ comme celles qui forment une congruence des sphères. Ceci est d'ailleurs évident d'après le fait que nous pouvons aussi définir $\left({ }^{2}\right)$ la circonférence généralisée comme une courbe dont le développement est une circonférence ordinaire et la correspondence entre $A_{\dot{n}}$ et $A_{\dot{n}}+d A_{\dot{n}}$ que nous avons établie dans le Chapitre 3 est une rotation conforme. Nous ppouvons choisir, de bien des manières, les sphères $A$ autre que $A_{\dot{n}}$ formant une telle congruence. Nous prenons, par exemple, les sphères tangentes à l'hypersurface et étant en contact du second ordre avec une courbe sur l'hypersurface, et cherchons les équations différentelles de la courbe autoconcourante. Une telle courbe peut être définie de la manière suivante:

Considérons une courbe sur notre hypersurface $S$ et désignons par $A_{\dot{0}}$ le point courant sur cette courbe. Soit $A(\sigma)$ la sphère qui est tangente à $S$ en un point $A_{0}$ de la courbe $u^{\alpha}(\sigma)$ et qui est en contact du second ordre avec cette courbe, et soit $k(\sigma)$ une circonférence sur $A$ qui est en contact $d u$ second ordre avec la courbe. En désignant par $u$ le paramètre sur la circonférence $k$, si l'on peut choisir les points $P(u)$ sur $k$ de telle manière que les trajectoires des $P(u)$ se coupent toujours orthogonalement avec la sphère $A$, on appelle cette courbe courbe autoconcourante spéciale.

La sphère $A$ étant tangente à l'hypersurface, nous avons

$$
A=\alpha A_{\dot{0}}+A_{\dot{n}}
$$

Le fait que $A$ est en contact du second ordre avec la courbe s'exprime par

$$
\left(\alpha A_{0}+A_{\dot{n}}\right) \frac{d^{2} A_{0}}{d s^{2}}=0
$$

(1) A. Haimovici (1) (2). (2) K. Yano (1) Y. Mutô (1). 
d'où

$$
\alpha=A_{\dot{n}} \frac{d^{2} A_{0}}{d s^{2}}=-\frac{d A_{\dot{n}}}{d s} \frac{d A_{0}}{d s}
$$

ce qui nous donne

$$
\alpha=a^{-\frac{1}{n-1}} M_{\alpha \beta} \frac{d u^{\alpha}}{d \sigma} \frac{d u^{\beta}}{d \sigma}
$$

La circonférence $k$ peut être exprimée par

$$
P=A_{0}+\frac{d A_{0}}{d s} u+\frac{1}{2}\left\{\frac{d^{2} A_{0}}{d s^{2}}+\frac{1}{2}\left(\frac{d^{2} A_{0}}{d s^{2}} \frac{d^{2} A_{0}}{d s^{2}}\right) A_{0}\right\} u^{2}, \quad P \cdot P=0,
$$

$u$ étant un paramètre sur la circonférence.

En substituant les équations

$$
\begin{aligned}
\frac{d A_{0}}{d s} & =\frac{d u^{i}}{d s} A_{i}, \\
\frac{d^{2} A_{0}}{d s^{2}} & =I I_{j k}^{0} \frac{d u^{j}}{d s} \frac{d u^{k}}{d s} A_{0}+a^{i} A_{i}+A_{\infty},
\end{aligned}
$$

dans (7.5), on obtient

$$
P=\left(1+\frac{1}{4} G_{j k} a^{j} a^{k} u^{2}\right) A_{0}+\left(\frac{d u^{i}}{d s} u+\frac{1}{2} a^{i} u^{2}\right) A_{i}+\frac{1}{2} u^{2} A_{\infty} .
$$

Cela étant, la condition pour que notre courbe soit autoconcourante peut être exprimée par

$$
\frac{d P}{d s}=\lambda P+\mu A
$$

$\lambda$ et $\mu$ étant convenablement choisis. Donc, en dérivant (7.6) le long de la courbe, on trouve

$$
\begin{gathered}
{\left[\frac{1}{4} \frac{d}{d s}\left(G_{j k} a^{j} a^{k}\right) u^{2}+\frac{1}{2} G_{j k} a^{j} a^{k} u \frac{d u}{d s}+\Pi_{j k}^{0}\left(\frac{d u^{j}}{d s} u+\frac{1}{2} a^{j} u^{2}\right) \frac{d u^{k}}{d s}\right] A_{0}} \\
+\left[\left(1+\frac{1}{4} G_{j k} c_{s}^{j} a^{k} u^{2}\right) \frac{d u^{i}}{d s}+a^{i} u+\frac{1}{2}\left(\frac{d a^{i}}{d s}+I \Pi_{j k}^{i} a^{j} \frac{d u^{k}}{d s}\right) u^{2}\right. \\
+\frac{d u^{i}}{d s} \frac{d u}{d s}+a^{i} u \frac{d u}{d s} \\
\left.+\frac{1}{2} \Pi_{\infty k}^{i} \frac{d u^{k}}{d s} u^{2}\right] A_{i}+\left(1+\frac{d u}{d s}\right) u A_{\infty}=\lambda P+\mu\left(A_{n}+\alpha A_{0}\right) .
\end{gathered}
$$


En comparant d'abord les coefficients de $A_{\infty}$, on trouve

$$
\frac{d u}{d s}=-1+\frac{1}{2} \lambda \cdot u \text {. }
$$

En multipliant ensuite $\left(\alpha+N^{0}\right) A_{n}+A_{\infty}$ à (7.8), on obtient

$$
\begin{gathered}
-\frac{1}{4} \frac{d}{d s}\left(G_{j k} a^{j} a^{k}\right) u^{2}-\frac{1}{2} G_{j k} a^{j} a^{k} u \frac{d u}{d s}-I I_{j k}^{0}\left(\frac{d u^{j}}{d s} u+\frac{1}{2} a^{j} u^{2}\right) \frac{d u^{k}}{d s} \\
+\left\{N_{i} a^{i} u+\frac{1}{2} N_{i}\left(\frac{d a^{i}}{d s}+I I_{j k}^{i} a^{j} \frac{d u^{k}}{d s}\right) u^{2}+N_{i} a^{i} u \frac{d u}{d s}+\frac{1}{2} I I_{j k}^{0} N^{j} \frac{d u^{k}}{d s}-u^{2}\right\}\left(\alpha+N^{0}\right) \\
-\left(\alpha+N^{0}\right) N^{0}\left(\frac{d u}{d s} u+u\right)=\lambda\left\{-1-\frac{1}{4} G_{j k} a^{j} a^{k} u^{2}+\frac{1}{2} N_{i} a^{i} u^{2}\left(\alpha+N^{0}\right)\right. \\
\left.-\frac{1}{2} u^{2}\left(\alpha+N^{0}\right) N^{0}\right\} .
\end{gathered}
$$

En substituant (7.9) dans cette équation, on a

$$
\begin{aligned}
\lambda= & \frac{1}{4} \frac{d}{d s}\left(G_{i k} a^{j} a^{k}\right) u^{2}-\frac{1}{2} G_{j k} a^{j} a^{k} u+\Pi_{j k}^{0}\left(\frac{d u^{j}}{d s} u+\frac{1}{2} a^{j} u^{2}\right) \frac{d u^{k}}{d s} \\
& -\frac{1}{2} N_{i}\left(\frac{d a^{i}}{d s}+I I_{j k}^{i} a^{j} \frac{d u^{k}}{d s}\right) u^{2}\left(\alpha+N^{0}\right)-\frac{1}{2} \Pi_{j k}^{0} N^{j} \frac{d u^{k}}{d s} u^{2}\left(\alpha+N^{0}\right) .
\end{aligned}
$$

Multiplions cette fois (7.8) par $\left\{A_{j}-\left(A_{\dot{n}} A_{j}\right) A_{\dot{n}}\right\} G^{i j}$, alors, on obtiendra

$$
\begin{aligned}
{\left[\left\{1+\frac{1}{4}\left(G_{j k} a^{j} a^{k}\right) u^{2}\right\} \frac{d u^{i}}{d s}+a^{i} u\right.} & +\frac{1}{2}\left(\frac{d a^{i}}{d s}+I I_{j k}^{i} a^{j} \frac{d u^{k}}{d s}\right) u^{2} \\
& +\frac{d u^{i} \frac{d u}{d s}+a^{i} u \frac{d u}{d s}}{d s} \\
\left.+\frac{1}{2} I I_{\infty \circ}^{i} \frac{d u^{k}}{d s} u^{2}\right]-N^{i}\left[N_{j} a^{j} u\right. & +\frac{1}{2} N_{j}\left(\frac{d a^{j}}{d s}+I I_{k h}^{j} a^{k} \frac{d u^{h}}{d s}\right) u^{2} \\
& \left.+N_{j} a^{j} u \frac{d u}{d s}+\frac{1}{2} I I_{j k}^{0} N^{j} \frac{d u^{k}}{d s} u^{2}\right] \\
+N^{i} N^{0} u\left(1+\frac{d u}{d s}\right) \quad & \lambda\left[\frac{d u^{i}}{d s} u+\frac{1}{2} a^{i} u^{2}-\frac{1}{2} N^{i} N_{j} a^{j} u^{2}+\frac{1}{2} N^{0} N^{i} u^{2}\right] .
\end{aligned}
$$


Les valeurs de $\frac{d u}{d s}$ et de $\lambda$ étant substituées, (7.11) deviennent

$$
\begin{aligned}
& \frac{1}{4} G_{j k} a^{j} a^{k} \frac{d u^{i}}{d s} u^{2}+\frac{1}{2}\left(\frac{d a^{i}}{d s}+I I_{j k}^{i} a^{j} \begin{array}{c}
d u^{k} \\
d s
\end{array}\right) u^{2}+\frac{1}{2} I I_{\circ k}^{i} \frac{d u^{k}}{d s} u^{2} \\
& -N^{i}\left\{\frac{1}{2} N_{j}\left(\frac{d a^{j}}{d s}+I I_{k h}^{j} a^{k} \frac{d u^{h}}{d s}\right) u^{2}+\frac{1}{2} I I_{j k}^{0} N^{j} \frac{d u^{k}}{d s} u^{2}\right\} \\
& =\frac{1 d u^{i} u\left\{\frac{1}{4} d s\right.}{4}\left(G_{j k} a^{j} a^{k}\right) u^{2}-\frac{1}{2} G_{j k} a^{j} a^{k} u+I I_{j k}^{0} \frac{d u^{j}}{d s} \frac{d u^{k}}{d s} u \\
& +\frac{1}{2} I I_{j k}^{0} a^{j} \frac{d u^{k}}{d s} u^{2} \\
& \left.-\frac{1}{2} N_{j}\left(-\frac{d a^{j}}{d s}+I I_{k h}^{j} \alpha^{k} \frac{d u^{h}}{d s}\right) u^{2}\left(\alpha+N^{0}\right)-\frac{1}{2} I I_{j k}^{0} N^{j} \frac{d u^{k}}{d s} u^{2}\left(\alpha+N^{0}\right)\right\}
\end{aligned}
$$

Ces équations doivent être satisfaites par n'importe quelle valeur de $u$, donc on tire de (7.12)

$$
\begin{array}{r}
\frac{d a^{i}}{d s}+I I_{j k}^{i} a^{j} \frac{d u^{k}}{d s}+\left(G_{j k} a^{j} a^{k}-I I_{j k}^{0} \frac{d u^{j}}{d s} \frac{d u^{k}}{d s}\right) \frac{d u^{i}}{d s}+I I_{\circ k}^{i} \frac{d u^{k}}{d s} \\
-N^{i}\left\{N_{j}\left(\frac{d a^{j}}{d s}+I I_{k h}^{j} a^{k} \frac{d u^{h}}{d s}\right)+I I_{j k}^{0} N^{j} \frac{d u^{k}}{d s}\right\}=0
\end{array}
$$

et

$$
\begin{gathered}
1 \frac{d}{d s}\left(G_{j k} a^{j} a^{k}\right)+I I_{j k}^{0} a^{j} \frac{d u^{k}}{d s}-N_{j}\left(\frac{d a^{j}}{d s}+I I_{k h}^{j} a^{k} \frac{d u^{k}}{d s}\right)\left(\alpha+N^{0}\right) \\
-I I_{j k}^{0} N^{j} \frac{d u^{k}}{d s}\left(\alpha+N^{0}\right)=0
\end{gathered}
$$

Mais l'équation (7.14) peut être déduite de (7.13) grâce à la relation $\alpha+N^{0}=N_{\alpha,} \frac{d u_{\alpha}}{d s} \frac{d u_{\beta}}{d s}$, donc les équations (7.13) sont les équations différentielles de la courbe autoconcourante.

Les équations (7.13) peuvent être écrites aussi sous la forme suivante

$$
\begin{aligned}
B_{\cdot i}^{\alpha}\left[\frac{d a^{i}}{d s}+I_{j k}^{i} a^{j} \frac{d u^{k}}{d s}\right. & +\left(G_{j k} a^{j} a^{k}-I_{j k}^{0} \frac{d u^{j}}{\lambda_{s}} \frac{d u^{k}}{d s}\right) \frac{d u^{i}}{d s} \\
& \left.+I_{\infty k}^{i} \frac{d u^{k}}{d s}\right]=0
\end{aligned}
$$


et on voit bien ici que la circonférence généralisée de l'espace ambiant est une courbe autoconcourante si elle se trouve sur l'hypersurface. Si l'on écrit (7.13) avec les grandeurs de l'hypersurface, on obtient

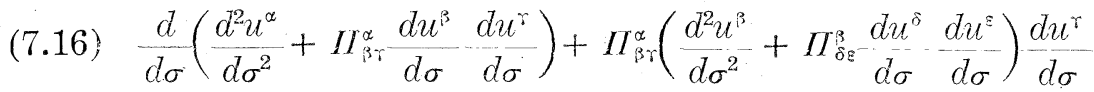

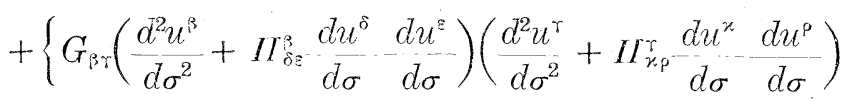

$$
\begin{aligned}
& \left.-I_{\beta r}^{0} \frac{d u^{\beta}}{d \sigma} d u^{\Upsilon}\right\} \frac{d u^{\alpha}}{d \sigma} \\
& +I_{\infty \beta}^{\alpha} \frac{d u^{\beta}}{d \sigma}-M_{\gamma \delta} \frac{d u^{\gamma}}{d \sigma} \frac{d u^{\delta}}{d \sigma}\left(M_{\cdot \beta}^{\alpha} \frac{d u^{\beta}}{d \sigma}-M_{\varepsilon x^{\prime}} \frac{d u^{\S} d u^{\kappa}}{d \sigma} \frac{d u^{\alpha}}{d \sigma} \frac{d \sigma}{d \sigma}\right)=0 .
\end{aligned}
$$

Chapitre 8. Les propriétés des ombilics et les hypersurfaces totalement ombiliquees.

Un point sur l'hypersurface où l'équation

$$
M_{\alpha \beta}=0
$$

est satisfaite s'appelle obmilic de l'hypersurface. Ceci est une propriété invariante par rapport à la transformation conforme: Or, nous avons, en un ombilic,

par suite

$$
d A_{\dot{0}} \cdot d A_{\dot{n}}=0
$$

$$
\left(d^{2} A_{0}\right) A_{\dot{n}}=0
$$

donc, la sphère $A_{\dot{n}}$ est en contact du second ordre avec l'hypersurface.

Pour les ombilics de l'hypersurface, nous avons une suite de théorèmes.

On peut, pour la démonstration, consulter un Mémoire de Y. Mutô (2) qui traite la théorie des sous-espaces dans un espace à connexion conforme.

Théorème: Si toutes. les courbes sur l'hypersurface passant par un point fixe et étant en contact du troisième ordre avec les circonférences généralisées de l'espace ambiant peuvent être en contact du troisième ordre avec une circonf'erence généralisée sur l'hypersurface, ce point est un ombilic.

Ceci s'ensuit immédiatement de (6.14). 
Théorème: Une condition nécessaire et suffisante pour que toutes les courbes autoconcourantes spéciales passant par un point fixe soient en contact du troisième ordre avec les circonférences généralisées de l'hypersurface est que ce point soit un ombilic.

Ceci se prouve facilement en raison de (7.16).

Une hypersurface dont tous les points sont ombilics s'appelle une hypersurface totalement ombiliquée. Pour les hypersurfaces totalement ombiliquées, nous avons les théorèmes suivants :

Théorème: Sur une hypersurface totalement ombiliquee, deux paramètres projectifs d'une courbe sur l'hypersurface l'un et l'autre étant définis en regardant la courbe comme étant celle de l'espace ambiant et comme étant celle sur l'hypersurface respectivement coüncident.

Théorème: Sur une hypersurface totalement ombiliquée, la circonférence généralisée et la courbe autoconcourante spéciale coüncident, inversement si ces deux courbes coüncident toujours, cette hypersurface doit être totalement ombiliquée.

Théorème: S'il existe toujours une hypersurface totalement ombiliquée passant par un point fixe et étant orthogonale à n'importe quelle direction, le tenseur conforme de courbure de $M$. Weyl s'annule en ce poin'. $\left.{ }^{1}\right)$

\section{Bibliographie}

E. Cartan

P. C. Delens

A. Haimovici

Y. Mutô.

S. Sasaki

J. A. Schouten

K. Yano
(1) Les espaces à connexion conforme. Annales de la Soc. Polonaise de Math. Tome 2 (1923) 171--221.

(1) Méthodes et problèmes en géométries différentielles euclidienne et conforme. Thèse, Paris, (1927).

(1) Directions concourantes le long d'une courbe sur une surface d'un espace conforme. C. R. Roumanie. Tome 1 (1937) 296-301.

(2) Directions concourantes et directions parallèles sur une variété d'un espace conforme. Thèse, Jassy, (1938).

(1) On the generalized circles in the conformally connected manifold. Proc. Imp. Acad. Tokyo. Vol. 15 (1939) 23-26.

(2) On some properties of hypersurfaces in a conformally connected manifold. Proc. Physico-Math. Soc. Japan. Vol. 21 (1939) 615-625.

(1) On the theory of surfaces in a curved conformal space. Science Report of the Tôhoku. Imp. Univ. Series I. Vol. 28 (1940) 261-285.

(1) Über die konforme Abbildung n-dimensionaler Mannigfaltigkeit mit quadratischer Massbestimmung auf eine Mannigfaltigkeit mit euklidischer Massbestimmung. Math. Zeitschr. Band. 2(1921) 58-88.

(1) Sur les circonférences généralisées dans les espaces à connexion conforme. Proc. Imp. Acad. Tokyo. Vol. 14 (1938) 329-332.

(2) Sur la théorie des espaces à connexion conforme. Journal of the Faculty of Science Imperial Univ. of Tokyo. Section 1. Vol. 4. Part. 1. (1939) 1-59.

(3) Sur les équations de Gauss dans la géométrie conforme des espaces de Riemann. Proc. Imp. Acad. Tokyo. Vol. 15 (1939) 247-252.

(4) Sur les équations de Codazzi dans la géométrie conforme des espaces de Riemann. ibidem. Vol. 15 (1939) 340-344.

(1) J. A. Schouten (1), K. Yano (4). 